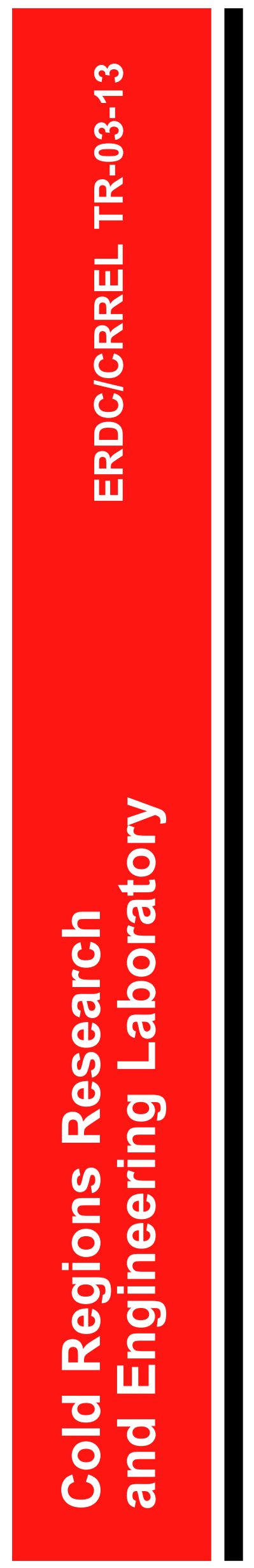

\title{
Solar Flux Initialization Schemes for Distributed Surface Energy Budget Modeling
}

George G. Koenig and David H. Tofsted

August 2003 


\section{Solar Flux Initialization Schemes for Distributed Surface Energy Budget Modeling}

George G. Koenig and David H. Tofsted

Cold Regions Research and Engineering Laboratory

72 Lyme Road

Hanover, New Hampshire 03755

Approved for public release; distribution is unlimited. 


\section{ABSTRACT}

It is a well-established fact that the state of the ground is driven in a large part by the downwelling solar and IR fluxes. Models developed to predict the state of the ground depend critically on these fluxes for initialization. When measured solar and infrared fluxes are not available they must be computed. We have compared the ground temperatures as computed by the thermal model SWOETHERM using different solar flux initialization schemes. These initialization schemes used measured solar flux values obtained during the Smart Weapons Operability Enhancement (SWOE) field programs, and calculated solar flux values from a semi-empirical model (Shapiro's model), a plane parallel model (MODTRAN), and ARL's AIM (Atmospheric Illumination Module) model. We investigated the response of the surface temperature to different solar flux initialization schemes while all other environmental parameters were held constant. We found that for clear skies all schemes resulted in nearly identical surface temperatures. For partly cloudy and cloudy skies only the AIM model can mimic the spatial variability observed with the measured solar fluxes. The Cloud Scene Simulation Model (CSSM) was used to determine the spatial variability of the clouds. The cloud distributions were then used by AIM to produce the variations of the surface solar loading. CSSM also has the capability to produce the temporal variations in the cloud fields for short periods of time. Thus, it would be possible to use CSSM and AIM to produce the temporal and spatial variations in the solar loading. Models like AIM frequently incur a large computational burden. In order to reduce the computational burden associated with AIM we have implemented several new procedures. Distributed energy budget models used to predict the state of the ground require distributed environmental parameters for initialization. Many of these parameters can be obtained from mesoscale models such as MM5 or databases associated with programs such as IMETS. But, to our knowledge, none of these models or programs provide distributed solar or infrared fluxes, a key initialization parameter of energy budget models. Models like AIM linked to CSSM, or for that matter any model that provides information on the spatial and temporal distribution of atmospheric conditions, can be used to provide the spatial and temporal distribution of radiative fluxes.

DISCLAIMER: The contents of this report are not to be used for advertising, publication, or promotional purposes. Citation of trade names does not constitute an official endorsement or approval of the use of such commercial products. All product names and trademarks cited are the property of their respective owners. The findings of this report are not to be construed as an official Department of the Army position unless so designated by other authorized documents.

DESTROY THIS REPORT WHEN IT IS NO LONGER NEEDED. DO NOT RETURN TO THE ORIGINATOR. 


\section{CONTENTS}

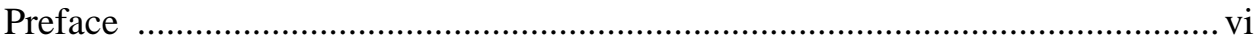

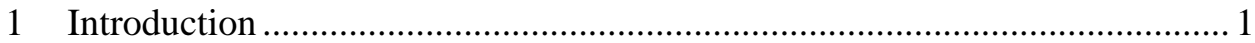

2 Solar Radiation Initialization Methods...................................................... 3

AIM surface radiation calculations ............................................................. 3

AIM model enhancements for AMIP ........................................................ 4

Semi-empirical surface radiation calculations............................................. 19

Atmospheric transmission model: MODTRAN …......................................... 22

Measured solar flux values..................................................................... 22

3 Thermal (SWOETHERM) Model ............................................................... 24

Soil/snow thermal model .............................................................................. 24

Vegetation thermal model .......................................................................... 24

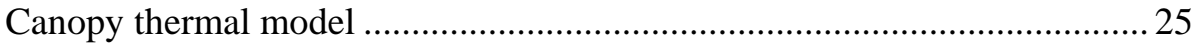

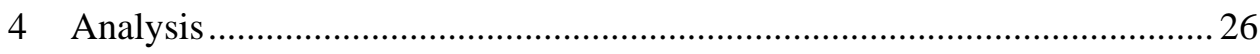

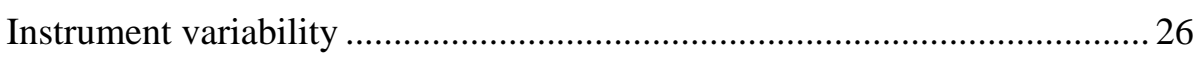

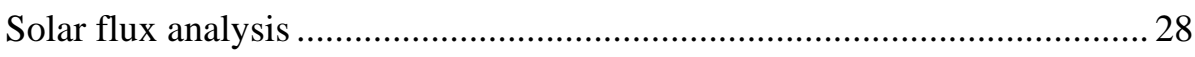

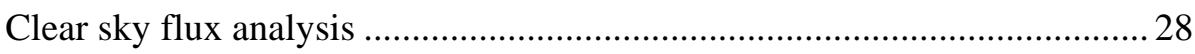

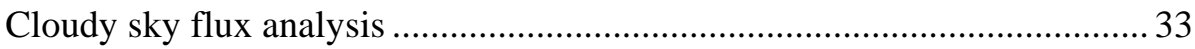

Partly cloudy sky flux analysis................................................................... 46

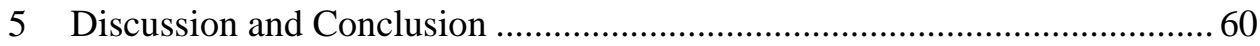

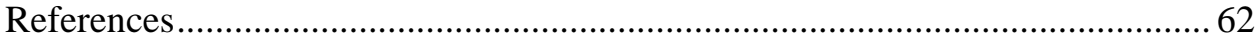

\section{ILLUSTRATIONS}

Figure 1. Comparison of co-located 8-48s and PSP for clear-sky

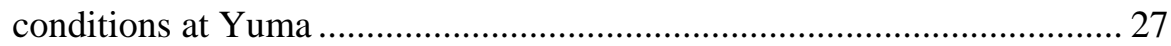

Figure 2. Comparison of 14 PSP for clear-sky conditions during Grayling II .. 27

Figure 3. Clear-sky flux, both measured and modeled, based on meteorological conditions from Grayling I field program......................... 29

Figure 4. Change in solar flux components as function of visibility as computed using AIM model ................................................................. 30 
Figure 5. Calculated surface temperature values for bare ground and different solar initialization schemes .......................................................... 33

Figure 6. Distribution of total solar flux at 1300 local as calculated

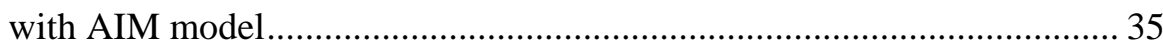

Figure 7. Histogram of AIM fluxes for 1300 local and measured fluxes .......... 36

Figure 8. Total solar fluxes for overcast conditions...................................... 37

Figure 9. Surface temperature for bare soil calculated using different

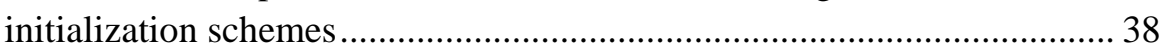

Figure 10. Comparison of different model computed flux and measured flux for cloudy conditions for Yuma .....................................................................4 40

Figure 11. AIM maximum fluxes for Yuma cloudy skies day ......................... 41

Figure 12. Surface temperature for bare ground using different initialization schemes

Figure 13. Total solar flux values for overcast low cloud conditions at Grayling II field site 43

Figure 14. Comparison of direct and diffuse measured and AIM-generated

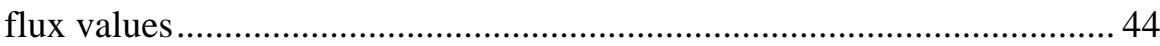

Figure 15. Histogram of total solar flux for 6400 AIM values and 50 measured values 44

Figure 16. Impact of different flux initialization schemes on bare-ground

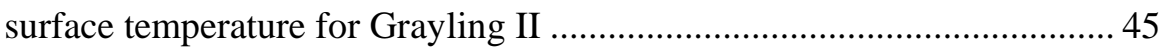

Figure 17. Solar flux and surface temperature for site D, 27 March, Yuma...... 47

Figure 18. Surface temperature and solar flux ratio for sites $C$ and D.............. 48

Figure 19. Distribution of total solar flux for sites E1 and E2 for Grayling I and AIM-calculated values 49

Figure 20. Surface temperature for bare ground resulting from different solar

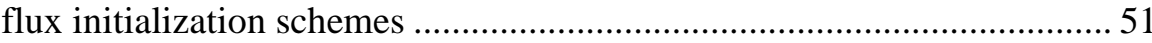

Figure 21. Distribution of total solar flux at 1300 local as calculated

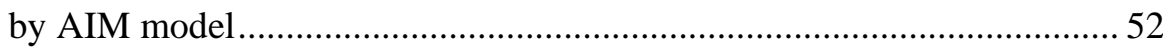

Figure 22. Mean AIM-calculated total solar flux \pm one standard deviation ...... 53

Figure 23. Surface temperature for medium vegetation (grass) for different

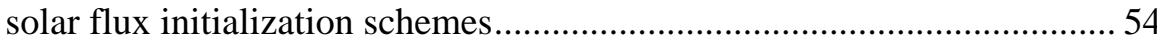

Figure 24. Total solar flux and surface temperature measurements at site E3

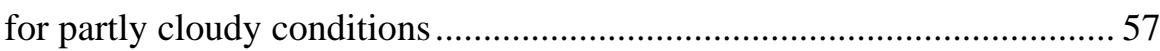

Figure 25. Distribution of measured total solar flux for period of 60 minutes for Grayling II and AIM-calculated values for 8- by 8-km area at 1000 local 57

Figure 26. Total solar flux measured and calculated for partly cloudy conditions for Grayling II ....................................................................... 58

Figure 27. Calculated surface temperature based on different initialization schemes 


\section{TABLES}

Table 1. Surface temperature differences for different surface types and different solar flux initialization schemes for clear-sky conditions for Grayling I

Table 2. Surface temperature differences for different surface types and different solar flux initialization schemes for clear-sky conditions

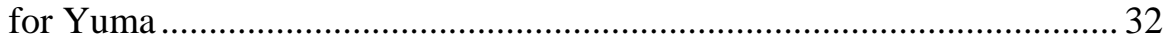

Table 3. Surface temperature differences for different surface types and different solar flux initialization schemes for clear-sky conditions for Grayling II

Table 4. Surface temperature differences computed using different solar flux initialization schemes and surface conditions 39

Table 5. Summary of impact of different solar flux initialization schemes on surface temperature for Yuma.

Table 6. Summary of surface temperature differences computed using different solar flux initialization schemes and surface conditions

Table 7. Cloud amounts used in solar flux models for partly cloudy conditions for Grayling I.....

Table 8. Summary of surface temperature differences computed using different solar flux initialization schemes and surface conditions ............. 51

Table 9. Cloud amounts used in solar flux models .............................................. 52

Table 10. Summary of surface temperature differences computed using different solar flux initialization schemes and surface conditions .............. 55

Table 11. Cloud amounts used in solar flux models ....................................... 56

Table 12. Summary of surface temperature differences computed using different solar flux initialization schemes and surface conditions 


\section{PREFACE}

This report was prepared by Dr. George G. Koenig, Research Physical Scientist, Geophysical Sciences Branch, U.S. Army Engineer Research and Development Center (ERDC), Cold Regions Research and Engineering Laboratory

(CRREL), Hanover, New Hampshire, and by David H. Tofsted, Physicist, Army Research Laboratory, White Sands Missile Range, New Mexico.

The work described in this report was funded by the Army Modeling and Simulation Office. The authors appreciate the technical review provided by Robert Davis and Richard Shirkey.

This publication reflects the personal views of the authors and does not suggest or reflect the policy, practices, programs, or doctrine of the U.S. Army or Government of the United States. The contents of this report are not to be used for advertising or promotional purposes. Citation of brand names does not constitute an official endorsement or approval of the use of such commercial products.

The Commander and Executive Director of the Engineer Research and Development Center is Colonel James R. Rowan, EN. The Director is Dr. James R. Houston. 


\title{
Solar Flux Initialization Schemes for Distributed Surface Energy Budget Modeling
}

\author{
GEORGE G. KOENIG AND DAVID H. TOFSTED
}

\section{INTRODUCTION}

It is a well-established fact that the state of the ground is driven in large part by downwelling solar and IR fluxes. Models developed to predict the state of the ground for Army operations will depend critically on these fluxes for initialization. Unfortunately, these fluxes are not routinely measured parameters as is the case with more common meteorological parameters such as temperature and relative humidity, so indirect methods must be used to generate the required flux initialization information for state-of-the-ground models. One-dimensional models used in a distributed mode to predict the state of the ground over some spatial domain require distributed initialization parameters over those domains. Assuming the atmosphere overlying the spatial domain of interest is horizontally homogeneous may lead to erroneous results. This would be especially true of partly cloud sky conditions where the solar loading can vary significantly over fairly small spatial extents. Davis and his colleagues (1998) have developed surface segmentation techniques based on terrain topography (slope and aspect) and land surface characteristics (soil type, vegetation cover, etc.). They found that segmenting a 1- by 1-km area at Grayling, Michigan, into 250 regions and running a one-dimensional thermal model for each region enabled them to accurately reproduce the spatial and temporal distribution of the snow cover. Mesoscale models such as MM5 have better forecast skills at 12 and $36 \mathrm{~km}$ than at higher resolutions. A resolution of $12 \mathrm{~km}$ may be insufficient for the initialization of distributed state-of-the-ground models, especially for radiative flux parameters and segmentations on the scale that Davis found to be optimal. This raises the question of the importance of using distributed initialization parameters for distributed modeling of the state of the ground. We will investigate the sensitivity of the ground temperature, a state-of-the-ground parameter, as predicted by the SWOETHERM thermal model to different flux initialization techniques, 
including initialization using measured flux values obtained during the Smart Weapons Operability Enhancement (SWOE) field programs.

The Cold Regions Research and Engineering Laboratory (CRREL) has numerous data sets that can be used to initialize the solar flux models. One of the more comprehensive ones was collected during the JT\&E SWOE program. These data sets also contain the information that can be used as ground truth for the evaluation of predicted solar fluxes. Solar fluxes will be calculated using a semiempirical scheme developed at CRREL based on the work of Shapiro (1972, 1982, 1987), a plane-parallel scheme using MODTRAN, and ARL's AIM (Atmospheric Illumination Module) model. Only the AIM model produces distributed flux values over a user-defined spatial domain. AIM uses the Cloud Scene Simulation Model (CSSM) (Cianciolo and Rasmussen 1996) in conjunction with the Boundary Layer Illumination and Transmission Simulation (BLITS) radiative transfer program to determine the spectral and spatial distribution of fluxes in cloudy and clear atmospheres. Unlike the first two approaches that are either a parameterization or assume a plane-parallel atmosphere, BLITS uses a physics-based approach that models three-dimensional fluxes through dense clouds. Scenarios will be run using data sets from Yuma and Grayling for different sky conditions (clear, partly cloudy, and overcast). 


\section{SOLAR RADIATION INITIALIZATION METHODS}

\section{AIM surface radiation calculations}

The Atmospheric Illumination Module has been extended to permit surface solar loading calculations in addition to basic surface illumination calculations. AIM was originally an extension of previous work accomplished as part of the ARL WAVES (Weather and Atmospheric Visualization Effects for Simulations) program to permit better computation of propagation and illumination effects in the presence of dense clouds. The AIM propagation model BLITS leveraged off useful properties of the WAVES model BLIRB (Boundary Layer Illumination and Radiation Balance), using the discrete ordinates method (DOM) for characterizing angular integration and scattering effects due to atmospheric haze aerosols. However, BLIRB did not perform well when dense aerosols (cumulus or stratus-type clouds) were present in the modeling volume. Thus BLIRB could not adequately characterize partly cloudy conditions. The BLIRB model had been designed to divide the modeled volume (typically a volume several kilometers deep starting at the earth's surface) into a series of rectilinear cells. In three dimensions (3-D), these cells were then populated with haze and cloud aerosols. BLITS uses this same technique, plus an improved technique for representing the scattering properties of cloud aerosols: EOSAEL's CLTRAN (CLoud TRANsmission) Khrgian-Mazin model for cloud aerosols and its predictions of vertical structure of aerosol density and properties within a cloud layer. In addition, a logleast-squares technique was adopted to determine the Legendre expansion coefficient used for representing the cloud aerosols input parameters to the radiative transfer model BLITS. Due to the modifications related to the new input requirements of the BLITS model, a front-end input processor was needed to provide realistic interpreted data to the BLITS model. This input engine is the AIM processor. In addition to various input processing routines, AIM was originally designed to process the outputs of BLITS into comprehensible and compressed output formats that would follow the same structure as previous WAVESdeveloped output formats. Thus, other WAVES codes could be adopted that used the WAVES FastVIEW output formats.

The FastVIEW format permitted visualization of cloud scenes by combining both extinction and limiting path radiance information in a single file. Two such visualization algorithms were developed that were compatible with outputs from either AIM or WAVES. The first was designed as an orthographic viewing technique in which all lines of sight through the modeled volume were treated 
using parallel lines. The second technique produced a perspective view of the modeled volume, where a mathematical pinhole camera model was used to construct lines of sight, all converging on the camera position, through the pinhole "aperture" and on to an image plane within the system (O'Brien and Tofsted 1998). To construct the appropriate input data sets to run these models, the original WAVES output data sets had to be expanded in number. Originally WAVES produced two data sets comprising a gridded surface illumination output file and a FastVIEW output file of gridded 3-D extinction and directionally varying limiting path radiance information. A third output file consisting of highresolution (62.5-m resolution vs. the standard 250-m resolution) extinction data was added because, when visualized, the 250-m FastVIEW data appeared as large illuminated blocks. Using the higher-resolution extinction data, along with interpolation methods applied to the coarser resolution FastVIEW radiance data, realistic visualizations of cloud features and illumination could be produced.

\section{AIM model enhancements for AMIP}

The goal of the AMIP program from the perspective of the AIM models was to expand the AIM modeling capabilities to allow surface solar loading calculations as a user option and to permit these calculations to be passed along to other codes in the form of solar loading databases. These databases were to include information on direct radiation (unscattered radiation directly from the solar disk) as well as diffusely scattered radiation. The goal of this division was to permit the directionally dependent illumination of various objects within a terrain/ surface model. Using this information, one would be able to differentially illuminate portions of the terrain, depending on surface self-shadowing features or surface directional orientations such as terrain slopes and/or complex vegetation surfaces. The data sets of surface illumination created are 2-D in nature, exhibiting variations in position due to overlying clouds and their shadows. Thus, issues such as the time-dependent changes in solar loading and the impact on the terrain temperature, and how these variations affect target/background signatures, can be addressed. To support this increasing role of AIM, a series of changes and/or additions were made to AIM. The following sections deal with the nature and implications of these changes.

\section{Correlated K-distribution technique}

The solar radiation that provides the primary heat source for the earth's surface consists of visible $(0.35-0.75 \mu \mathrm{m})$ and near-infrared (NIR) $(0.75-4.0 \mu \mathrm{m})$ energy. Most of the energy at the shorter wavelengths (UV) is absorbed by ozone in the upper atmosphere, and because AIM focuses on tropospheric effects, these 
absorption effects are largely accounted for by initializing the AIM models with the MODTRAN code. However, across the NIR spectrum there exist several regions where water vapor absorption is important. Concerns over propagation effects across these band structures are the motivating factor in moving from a series of sampled absorption effects used previously in AIM-visible band calculations to the integrated solar loading calculations needed for this project.

The AIM models are based on use of Beer's law, which assumes monochromatic transmission effects (negative exponential function of the optical depth traversed). Unfortunately, when considering various absorption bands in the NIR, the optical depth can vary greatly over a given band. Brute force methods simply increase the number of spectral channels used to accommodate the increased variability in extinction coefficients. But this technique is possible only in models that make concessions in accuracy elsewhere, such as using a lower spatial dimensionality. That is, the model atmospheres are not allowed to vary horizontally. To permit horizontal variations in a model requires commensurate increases in processing time because the computational difficulty is proportional to the number of processing cells used. The 250-m resolution typically used within BLITS/BLIRB, though rather coarse from a visualization perspective, nevertheless captures typical minimum cloud dimensions. Yet even this size cell imposes significant computational burdens.

The problem was how to permit both the integration over the full solar spectrum needed to compute the energy arriving at the surface that included horizontally inhomogeneous illumination conditions while simultaneously avoiding excessive computational times. The solution to these competing priorities came in the form of an adaptation to the correlated $k$-distribution method (Lacis and Oinas 1991, Fu and Liou 1992).

The correlated $k$-distribution method is an extrapolation of the $k$-distribution method. Under the $k$-distribution (KD) method, instead of representing the variations in the extinction coefficient $(k)$ as a function of wavelength, $k$ is given as a function of its probability distribution, the probability that $k$ will be less than a given value. Under these circumstances, the governing probability parameter $g$ will vary between 0 and 1 as $k$ varies between minimum and maximum values for a given spectral band in a given height interval above the surface. The major assumption of this technique is then invoked, whereby the extinction coefficient distribution functions at different levels in the atmosphere are assumed to be correlated. The $k$ distributions at each level can then all be parameterized through a single parameter $g$. Then, instead of evaluating the propagation characteristics as a function of wavelength, the same quantities can be evaluated using fewer intervals over the range of $k$ variations as functions of $g$. 
We took this correlation technique several steps further. The inputs required to evaluate irradiance values within the AIM program BLITS include information concerning the diffuse and direct radiation at the upper boundary of the BLITS modeled volume as well as layer extinction information. Thus, there are elements of the input set that correspond directly to data produced in the correlated $k$ distribution method as well as radiance information. But we would expect this radiance data to be somewhat correlated to the extinction data because highextinction regions should correspond with low-radiance regions if the energy is being removed in overlying layers due to the same absorption processes. In the typical AIM case, the modeled volume upper boundary is at $4 \mathrm{~km}$. There is ample atmosphere above $4 \mathrm{~km}$ in which water vapor effects absorb sufficient energy from the incident radiation to produce correlations between the incident radiation and the extinction coefficient data.

To assess these correlations, we had to alter the way the MODTRAN model was called in initializing the boundary conditions (at the 4-km boundary) for runs of the AIM BLITS model. Originally, for the visible light cases studied, MODTRAN was called only at a minimal sampling of wavenumbers. The visible band generally spans the wavenumber interval between 14,440 and $24,240 \mathrm{~cm}^{-1}$. The visible band was modeled as seven bands of $1400 \mathrm{~cm}^{-1}$ by sampling the spectral extinction and incident radiance at the ends of these bands using a $100-\mathrm{cm}^{-1}$ interval and averaging the maximum values. In the visible band, this approach appeared reasonable due to the low absorptivity of the atmosphere over each band. But in the calculation of the solar loading, this approach would be inappropriate over the NIR region due to band absorption problems. We could not greatly increase the number of equivalent bands used without greatly reducing performance, nor could we use the standard correlated $k$-distribution approach, which primarily applies to narrow band calculations. In fact, because our run mechanism relies on MODTRAN, which has a maximum resolution of only 1 $\mathrm{cm}^{-1}$, it is impossible to model the actual correlated $k$ distribution (CKD) because that calculation requires spectral resolutions on the order of anywhere between 20 and 2000 per inverse centimeter.

Instead, what was developed was an approximation to the correlated $k$ method. In this approximation, we first focused on transmission rather than the extinction coefficient because a band region that had a low transmission would be similar to another low-transmission result regardless of whether the extinction coefficients were off by an order of magnitude or more. The significant issue was not the absolute coefficient value but rather whether energy was transmitted or not, since we were primarily interested in the energy reaching the surface. Second, we needed to consider all the transmission results across the entire solar band at once because we had to reduce the number of channels to a minimum. 
Standard CKD methods tended to use narrow bands (perhaps as small as a few hundred wavenumbers in width), however, so we developed measures of compactness for sets of sample input data that would be used in dividing the data between the different channels.

The data required to run the BLITS model include an equivalent channel extinction coefficient for all molecular absorbers in each 1-km atmospheric layer from the model base to its top. In addition, values for the incident direct irradiance and diffuse radiances in a set of downward-streaming directions are needed for boundary input information at the volume top. In general, we use a 24-stream diffuse radiance model, requiring 12 downward diffuse stream calculations. When combined with the extinction data requirement and the direct radiance information, 14 MODTRAN model runs in all were required.

Under this AMIP program we looked at the problem of model initialization from two aspects. The first considered how best to provide data needed to initialize the thermal model, SWOETHERM, in order to provide a basis for model performance analysis. Considering this need, we wanted to determine the results for several case studies as quickly as possible, and although we would have preferred to develop a highly optimized code for rapid computation, it was not possible given the timetable required to provide the data. Therefore, we initially developed a technique for making the CKD computations in a way that required as few model alterations as possible. As a result of performing these initial calculations, however, we discovered that the processing times required to perform these KD computations were responsible for increasing the overall model computation time by approximately 50\%. Thus, after the case study data had been generated, we developed a second method for evaluating an equivalent set of MODTRAN-based boundary and initial condition data that will permit solar loading calculations but which would not require the direct running of MODTRAN at execution time. We have divided the comments regarding code improvement between this subsection, which only discusses the original CKD technique itself, and additional techniques under development to optimize the processing of the CKD data.

The CKD method adapted for this task involved, first, the development of a conceptual model of the input data required to run the radiative transfer model BLITS over the entire solar spectrum. In that model it was first realized that correlating 14 independent data variables (the 12 diffuse stream radiances, the direct stream radiance, and the four 1-km extinction coefficients) would be too much to attempt. Further, we knew that, in addition to the data produced by the MODTRAN runs, the frequency variable was also of interest because the aerosol scattering properties in the troposphere vary strongly with wavelength. We thus 
conceived a means of reducing the number of data elements to be correlated from effectively 14 down to only three. The method chosen was to interpret a single incident energy value for the net radiance crossing the volume upper boundary to represent the 12 diffuse channel elements plus the direct channel data. It was assumed that channel data undergoing similar extinction or scattering would exhibit similar forms for the net directional properties of the scattered energy. The results in radiance were then normalized such that the results varied between 0 and 1, representing the minimum and maximum points within the MODTRAN output set. A second variable reflected the average transmission of each 1-km layer. A third variable represented a normalized wavenumber parameter, which varied from 0 to 1 across the band of interest. These resulting three-element data sets were then envisioned as pointing to locations in a 3-D volume where wavenumber varied along one axis, the generalized incident radiation parameter along a second, and the transmission parameter along the third.

Running the MODTRAN algorithm at $2 \mathrm{~cm}^{-1}$ resolution over the solar spectrum produced a "cloud" of points within a unit cube once the results for each point were translated into their associated three parameter values of wavenumber, irradiance, and transmittance. A pattern recognition algorithm was then used to determine logical clusters of points within this cloud of sample values.

The method of running the pattern recognizer was relatively simple. First, all the points were divided into a minimal number of "classes" of points that were generally divided into wavebands according to visible color classes and a single NIR band. The mean values of each of the three parameters were then computed, and the class with the most variance about the means was divided into two daughter classes whose mean positions differed slightly from one another along the axis of the vector component with the greatest variance. Each sample point was then compared to the newly developed class centroids and new class assignments were determined, depending on which class centroid the point was closest to. Once all points were reassigned to a new class, using the class assignment value of each point, a new centroid could be computed for each class. Subsequently, the variance of points within each class, along each dimensional axis, could be computed. At each stage, the class with the greatest variance in any dimension was then divided into two new classes, and points belonging to the old classes would be reassigned between the new classes, depending on their proximity to the new centroids. Classes would continue to be divided until one of two conditions was satisfied: either a maximum (user-specified) number of classes had been reached, or (highly unlikely) all variances within all classes reached zero. In the case of our computations, since we would never be able to process a sufficient number of classes to eliminate all variance within the various computational spectral "channels," the best that could be achieved was to minimize the 
amount of variation over each channel. Overall, this pattern recognition technique is a variant of the well-known ISODATA algorithm (Ball and Hall 1965).

A major assumption of this approach was that variations within a single channel would tend to cancel, leading to overall realistic surface solar loading calculations, even though results for individual narrow-band constituents of a channel might show dispersion about that result. But this could occur only if the means used to determine the average statistics for the channel was chosen in such a way as to be connected to the mean associated with that channel. To ensure that class statistics were situated about the proper mean values, the wavelength and transmittance statistics were weighted according to the available incident energy at each individual wavelength interval. For example, it was discovered that the transmission and incident radiation results were positively correlated. That is, when we observed energy in a highly absorbing band there was usually less available (more had already been absorbed out of the incident energy at the top of the modeled volume) than in a nearby low-absorption region. Thus, it would not be correct to simply weight the transmission parameter evenly across a single output channel/class of data. More energy would be available for propagation through the low-absorption region, and thus, overall there would be a higher effective transmission capability than would be determined if properties of each wavelength interval were given equal weight. To implement this technique within AIM, the model execution stage was augmented by a solar loading option, which called a modified version of MODTRAN and included a call to the new classifier algorithm.

\section{Y2K compatibility issue}

In keeping with the concern over Y2K issues, the AIM models were adjusted to handle crossover into the next century. Previously, these codes had used an ephemeris computational engine that was destined to fail for dates beyond 1999, but this failure was primarily due to lunar position coefficients that were based on a 1977 effective date. Subsequently these codes were updated to permit unlimited computations into the future based on recomputation of the governing coefficients for the given year of the run being made. There remain, however, possible differences between the algorithms used by AIM and those used under SWOETHERM. The differences have not been completely accounted for, but appear small relative to solar loading concerns. They may be related to observer altitude issues. 


\section{SWOETHERM model interoperability}

To build in compatibility between the AIM outputs and the SWOETHERM inputs, we added an auxiliary routine to translate between the standard 250-m resolution surface information output file produced by AIM and a standard 100$\mathrm{m}$ format expected by SWOE. To provide this compatibility, we had the option of increasing the resolution of the AIM/BLITS radiative transfer model run or reformatting the output. We chose to reformat the data rather than run at higher resolution. This was a practical rather than an optimal decision. Using a $100-\mathrm{m}$ resolution grid within BLITS would have produced output databases 15.6 times larger than the current data sets, which typically occupy 300 MB of disk space. Such an expansion seemed unrealistic.

Instead, a cosine interpolation routine previously used for generating image overlays was adapted to produce 100 -m-resolution solar loading maps. These files contained information about direct, diffuse, and surface-emitted total radiation over the wavelength interval of interest. Of course, since there is little thermal radiation over the solar band, the emitted values were uniformly near zero. In addition, these files contained information about the horizontal dimensions of each cell and the overall domain of the modeled volume (standardized at $8 \mathrm{~km}$ in both the east-west and north-south directions). In addition, the reformatting technique transformed the order in which the results were output. The standard AIM output presented the data in columns running south to north as the inner loop. The SWOETHERM format required the data to be presented in terms of rows of data running east to west as the inner loop and with the columns presented in a north-to-south order.

\section{AIM model acceleration}

As mentioned above, in extending the AIM model to evaluate effects over the entire solar spectrum, MODTRAN had to be run at rather high spectral resolution $\left(2 \mathrm{~cm}^{-1}\right)$ and the results post-processed using the pattern recognition classifier based on correlated $k$-distribution theory. The unfortunate side effect of this procedure was that while we were able to provide AIM with the inputs it needed to run the BLITS model, the "cure" was rather costly. Running MODTRAN at high resolution took nearly as long as the combined running times of the remainder of the AIM codes. Thus, from a practical standpoint, the continued use of MODTRAN becomes problematic to the use of these modules in any realistic real-time or other Army operational application. However, if that purpose is ultimately to be achieved, it will be necessary to disconnect AIM or any future 3-D radiative processing techniques from MODTRAN or other highspectral-resolution processing routine. The issue is this: high-spectral-resolution 
codes assume a 1-D atmosphere that is simple to model and uses a coarse spatial resolution. The product of the spatial elements times the number of spectral channels is then roughly comparable for AIM and MODTRAN, but while we have control of AIM, permitting us to take shortcuts on the spatial side, MODTRAN must be preprocessed to eliminate its processing costs up front. While these costs are then translated into the storage space required to archive the resulting data sets, at least they would not impact processing time for the actual computations.

The overall goal of this computational engine is to generate any output product within a maximum of 5 minutes. Although we are not going to get to this point at the conclusion of this program, we are considerably closer than we were previously and, with the advent of faster computers with more memory, the prospect of achieving such performance or better within the next 10 years is highly likely. What follows then are the steps that were achieved during this program in terms of model speed increases.

The first performance enhancements were applied to the BLITS algorithm. Though these changes tended to slightly decrease the model accuracy (1-3\% greater error), the resulting model produces results that are still well below the uncertainties in several other model input variables such as cloud cover, cloud properties, haze layer thickness, and haze extinction properties. Two changes involved aspects of the BLITS main routine. First, the error-checking coefficients were increased significantly. Previously a point value difference between two iterations of $0.01 \%$ was considered sufficient to flag an item as an error. This condition criterion was increased by a factor of 20 , reducing the number of model iterations required to satisfy stability of the solution by approximately $30 \%$.

The second major change focused on a means of accelerating the algorithm convergence. Previously, BLITS simply iterated on the scattering source using the previous iteration's diffuse radiance terms as input to the current iteration's scattering equations and continued this iteration process until the diffuse results stabilized. However, this method was improved upon by using an acceleration technique operating on the results of two consecutive iterations. This technique requires additional memory to store a new copy of the diffuse stream array from an intermediate computation, but it permits the iteration procedure to converge with a third less iteration.

A final BLITS acceleration technique regarded a change in the means of initializing the effects of direct beam illumination on individual cells within the modeled volume. Previously, lines of sight were traced to sample points along the outer walls of each cell within the modeled volume. Statistical averages were then generated to describe overall illumination for each cell and for the overall 
direct energy scattered, absorbed, and transmitted within each cell. However, in cases where the sun was near the horizon, this technique became computationally intensive due to the horizontal periodicity normally employed in describing the edge effects. Under these conditions, for each point on an input cell surface a line of sight to the volume upper boundary could span many copies of the modeled volume prior to exiting the volume.

To accelerate this process, the results at each height level in the volume would be stored and used at the next lower level in the ray tracing process. For each new cell, interpolations on results for previously evaluated cells could be used so that a line of sight need go through no more than several cells at most. In many cases, results could be obtained by passing only through a single cell. This change led to slightly longer times for cases in which the sun was directly overhead, but much faster processing times for cases in which the sun was near the horizon. Given that the sun must be near the horizon at least twice each day, this change saves considerable processing time on average. There is a slight increase in the model error due to the cascading effects of interpolations on interpolations at each lower level. In some cases, these cascading effects are minimized (for small zenith angles), but for other cases [large zenith angles with low density (no cloud) atmospheres] the errors tend to be on the order of several percent.

\section{Eliminating direct MODTRAN runs}

The final acceleration method is dictated by the need to avoid direct MODTRAN runs in calculating solar loading. The reason for this need is obvious: MODTRAN must be run at high resolution to obtain information on the NIR absorption band structure that is critical in determining the total energy reaching the surface. However, this same accuracy requirement is a liability to rapid calculation of effects. Thus, direct calls to MODTRAN should be avoided. To eliminate them, it will be necessary to produce sets of preprocessed data representing characteristic MODTRAN outputs under a variety of conditions. Then interpolations can be made on the precomputed data sets.

The first step in this process was to understand the nature of the data space of MODTRAN outputs. Then a method of interpolation was developed that was extensible, given a variable-size output database. First, consider the size of the database. Tests were conducted where MODTRAN was exercised under a varying set of conditions. Output variables of interest were those required by AIM as input: extinction coefficients, direct irradiance, and diffuse radiance at the top of the AIM modeled volume. For purposes of these computations, AIM's volume was fixed at a 4-km vertical extent. Initially only solar source data sets were considered. Presumably, if the model's upper boundary was allowed to vary in height 
and a lunar source was permitted, then this would extend the database requirement by two additional dimensions. As it was, the extinction coefficients were found to vary only with respect to the vertical structure model selected (1976 U.S. standard atmosphere, midlatitude summer, etc.) and height of the surface above sea level. The solar direct radiation was found to vary with solar zenith angle in addition to the original two variables. The diffuse parameters showed additional dependence on the surface reflectivity and haze layer visibility.

Four of these data variables could be evaluated as floating-point numbers for interpolation purposes (zenith angle, surface reflectivity, visibility, and surface height above sea level). But the atmosphere-type parameter was provided to MODTRAN in terms of an integer. However, it was noticed that these "model" values associated with various atmospheric state scenarios (midlatitude summer, midlatitude winter, subarctic summer, subarctic winter, U.S. standard atmosphere, and tropical) could be described according to latitude and season, which could be expressed in terms of two additional parameters. The six main atmospheric scenarios could be associated with 2-D season/latitude data points and interpolations made with respect to a user-specified point within the grid. In one sense, then, these results would permit a user greater flexibility than was currently possible using the existing MODTRAN code, because what-if questions could be asked about intermediate conditions, while the previous model permitted only fixed choices of atmospheric models to be used.

It was decided that the resolution in each of the data variables was a significant consideration for interpolation purposes. That is, having too few cases could result in bad interpolations. Also, some variables had more dynamic range than others. For example, zenith angle could vary between 0 and $90^{\circ}$, but surface reflectivity could vary only between 0 and 1 . To account for these dynamics, different variables were resolved by different numbers of sample points. Zenith was varied between 0 and $88^{\circ}$ in increments of $11^{\circ}$. Visibility was varied from 1 km through $64 \mathrm{~km}$ in logarithmic fashion ( $2 \mathrm{~km}, 4 \mathrm{~km}, 8 \mathrm{~km}$, etc.). Reflectivity was varied linearly between 0.05 and 0.20 in increments of 0.05 ; it was then set to $0.3,0.5$, and 0.9 to handle cases of highly reflective surfaces (white sand beaches and/or snow-covered surfaces) albeit at lower resolution. The six unique model cases enumerated above were each computed separately, but categorized according to a scheme that assigns tropical results to a latitude of $10^{\circ}$, midlatitude results to latitude $40^{\circ}$, and subarctic results to latitude $70^{\circ}$. Since the tropical results are presumed not to be affected by seasonal variation, they are assigned to the tropical latitude regardless of season. The summer results, however, are assigned to the Julian date associated with the summer solstice, and similarly the wintertime results are assigned to the winter solstice. The U.S. standard atmosphere is then treated as a special case and assigned to the midlatitude equinox 
condition. Finally, the height above sea level of the model surface was ranged from 0 to $2 \mathrm{~km}$ in increments of $250 \mathrm{~m}$.

Overall, there are nine zenith positions, seven visibilities, seven reflectivities, six models, and nine altitudes, for a total of 23,814 cases to be computed under this archival approach. Obviously, it is not practical to archive the complete MODTRAN outputs of all these runs, nor would it be wise to await the completion of computing results for every case before beginning to attempt interpolations on a partial data set. It is estimated that the total processing time required to evaluate this 23,814 -case data set is over one year on a typical workstation. Instead, first, the CKD-processed channel data for a given case had to be computed and the resulting data stored for this case. And second, a method of interpolation had to be developed that could produce results for partial databases.

Let us consider the first aspect of this problem. Because we are interpolating our results between difference conditions, for which CKD results must be archived, the CKD method described previously, which optimizes the choice of channel assignment for each $2 \mathrm{~cm}^{-1}$ interval, cannot be used as-is in this archiving mode. The reason is the channel assignment technique. It would be highly likely (and it was noted in tests of the method) that similar (though not exact) conditions produced variable channel assignments. Thus any interpolation method used must rely on a means of archiving the MODTRAN results that regularizes the method of channel assignment. To do this, a separate version of the classifier algorithm was developed that read the choice of channel assignments from an external file. This file was from a run of the original model on what was considered a standard case: average solar zenith angle, average surface reflectivity, etc. The assignment data for this case were output and stored in a separate file that was then used as the template for channel assignments in all subsequent classifier calculations.

Because it might be possible to run the surface radiation calculations at varying resolution, the results of the MODTRAN runs were processed using variable numbers of channels: First, results were computed for the original sevenchannel visible scenario, then solar loading calculations were made with 7, 15, 20, and 25 channels. Each of these five scenarios is created using the output from MODTRAN runs over the solar band for every combination of the main five variables described above.

\section{Interpolating results using sample MODTRAN runs}

The major challenge associated with this technique is to perform meaningful interpolations based on less than a full set of sample case outputs. The problem is simple: given a total of 23,814 cases and a production rate of approximately 65 
cases per 24-hr period under optimal conditions, the database will require just over a year of continual processing time to evaluate, assuming no stoppages in production. What if the computational engine is halted temporarily? How can the order of computation be optimized such that interpolations can be as accurate as possible as soon as possible?

To accommodate computational interruptions, a simple technique was developed whereby a file listing all previously computed results is updated each time a new set is added. The interpolation method also refers to this listing in order to interpolate input conditions using results computed to date.

To optimize the order of the computations, a two-stage process was used. In the first stage, 48 data sets were calculated, which represented the outer shell of the most extreme conditions to be modeled. For the zenith angle, the 0 and $88^{\circ}$ cases were considered. This meant that, for the first four data elements, minimum and maximum conditions were considered. For the atmosphere-type parameter, the subarctic winter and summer conditions were considered, along with the tropical condition, for a total of three conditions, which mapped into five data sets (the tropical case maps into three cases total due to seasonal independence). The 48 computed cases thus map into a total of 80 cases.

Once this initial series of extreme cases were run, the processing code considered interior points using a weighting scheme. For each index position of each variable, a number between 0 and 4 was assigned, indicating a somewhat arbitrary assessment of the importance of evaluating results at that value of the variable in question. For example, for the ground altitude parameter, the $0 \mathrm{~m}$ above sea level condition was given highest priority (weight 4), followed by the 1000-m condition (weight 3 ), and then by the 500-m condition (weight 2), etc. The weights for each combination of run conditions are then added and a sum is obtained. The maximum sum of all the weights is 20 . The order in which computations are performed is determined by an iterative procedure. First, only conditions whose weight total sums to 20 are computed. Once all the possible combinations have been checked and all the 20 weight results have been computed, the algorithm cycles back again and computes all the cases whose weight total sums to 19. Following this iterative approach, presumably, the most important cases will be computed first. In this way the algorithm gradually fills in the set of computed results in a smooth manner and avoids the problems associated with systematically processing the model space in a standard iterative fashion that might leave large data gaps until late in the production process.

Given a database in a state of partial completion, what method of interpolation is best? To answer this question, we initially considered full interpolation over an array of nearby points, but found that this technique would not be able to 
account for gaps in the available data. The problem is balance: given a partially filled database, it is nearly impossible to build a window correctly around a point of interest that equally balances the influence of sample points on opposite sides of the point in question. Equally important are methods of dealing with points near the boundaries of the hypercube of possibilities.

For such cases it is important to develop a method that will not merely average results from nearby points. To perform an interpolation, let us assume the vector $\vec{x}_{j}$ is composed of vector components $x_{i, j}$, where $i$ cycles from 1 to 6 , representing the six different parameters (zenith angle, visibility, etc.), and where $j$ cycles from 1 to $J$, the number of sample data sets currently processed. As time increases, $J$ will increase, but at processing time this number will be fixed.

Associated with each point in the parameter space there would be a specific vector output of MODTRAN that describes the input needed to run AIM at a set of spectral channels. Let the elements of this vector $(\vec{Y})$ be given as components $\left(Y_{K}\right)$. Thus we have a space where components are functions of the point in the parameter space, or,

$$
\vec{Y}=\vec{f}(\vec{x})
$$

The problem is then to solve for $\vec{Y}$ given results $\vec{Y}_{j}$ at a finite set of sample points $\vec{x}_{j}$. The question is how best to accomplish this task.

Originally an approach was considered whereby a quadratic interpolation about a given point could be computed. However, the solution of this problem would involve high-order terms that could not be readily solved for, and the result could be unstable. Instead, a model was developed that was linear in each of the six parameters and centered about the point in question. That is, we assume,

$$
Y_{K}(\vec{x})=A_{K, 0}+\sum_{i=0}^{6} A_{K, i}\left(x_{i}-X_{i}\right) .
$$

$\vec{X}$ is the point in parameter space of interest, with the $X_{i}$ being the components of $\vec{X}$. The $A$ coefficients are produced as a result of a least squares analysis. There will be a separate set $(\vec{A})$ of coefficients for each element $Y_{K}$ of the solution vector.

By centering the solution about point $\vec{X}$, once the $A$ coefficients have been solved for through the least squares analysis, and since we are interested in the point $\vec{x}=\vec{X}$, we then have the simple solution, $Y_{K}(\vec{x})=A_{K, 0}$. Thus the 
formality of the least squares process is simply to remove any linear trends in the data such that the resulting estimate of the value will not contain any remaining linear bias.

We solve for the $A_{K}$ s using the available data $\left[\vec{Y}\left(\vec{x}_{j}\right)\right]$. However, we also need to account for the nature of the data set available. A normal least squares analysis would give each sample point equal weight. And while we would assume that the functional dependence of $\vec{Y}$ should be relatively slowly varying with any of the individual parameters, it may be that the overall dependence with some of the parameters may be nonlinear. In this case, giving equal weight to all sample points would be bad, because distant points would tend to reduce the estimate (for negative second derivative) or raise the estimate (for positive second derivative). Thus, it is necessary to weight the value of available data points such that linearities in the nearby region would receive greater weight.

For example, let the distance between the central point $(\vec{X})$ and a sample point $\left(\vec{x}_{j}\right)$ be measured as

$$
d_{j}=\sqrt{\sum_{i=0}^{6}\left(x_{i, j}-X_{i}\right)^{2}} .
$$

$i$ now varies between 0 (the constant term) and 6 . Now let the weighting scheme assume the form

$$
W_{j}=\exp \left(-\frac{d_{j}^{2}}{\sigma^{2}}\right)
$$

where $W_{j}$ is the weight assigned to the $j$ th sample and $\sigma$ is a width parameter that is fitted to the distribution of computed data sets about the point of interest, $\vec{X}$. Given the weighting scheme above, let us define the sum-squared error as

$$
\Delta_{K}=\sum_{j=1}^{J} W_{j}\left[Y_{K, j}-A_{K, 0}-\sum_{i=0}^{6} A_{K, i}\left(x_{i, j}-X_{i}\right)\right]^{2}
$$

where $\Delta_{K}$ is the error in the $K$ th element of $\vec{Y}$.

From a theoretical point of view, this approach assumes that each parameter is independent of all others. Obviously this leads to calculations that make no sense, such as solar zenith angles of $0^{\circ}$ at subarctic latitudes, but as long as $\sigma$ is kept sufficiently small, this should not lead to significant errors.

Using the least squares analysis approach, we take the partial derivative of $\Delta_{K}$ with respect to one of the variables $A_{K, i}$. The resulting problem requires the 
solution of a matrix multiplication, $\vec{B}=\overrightarrow{M A}$, where $\bar{M}$ is a square matrix composed of correlation elements.

Although the solution of this matrix problem, involving the matrix inverse of $\bar{M}$, is fairly routine, there remains the issue of determining the width parameter, $\sigma$, given a particular arrangement of nearby sample points. If $\sigma$ is set to too large a value, faraway points may have too significant an influence on the final results. For example, if there are numerous nearby points already available, it makes little sense to permit distant points to have much bearing on the outcome. On the other hand, if there are few nearby points, it may be necessary to increase $\sigma$ in order to ensure a statistical sample that is meaningful. The question is, how should $\sigma$ be determined?

Compounding this problem is the fact that we are not dealing with a "normal” three-dimensional space. Rather, the interpolation space is six-dimensional in nature. And since $\sigma$ is used to weight the total distance in any combination of dimensions, we need to know the hypervolume of a hypersphere of radius $\sigma$ in 6-D space to be able to determine how dense the set of sample points is in the neighborhood of $\vec{X}$. To determine the functional form of this space, a pointcounting algorithm was developed. Using this method it was determined that a sphere of radius $r$ in six dimensions has a hypervolume of $V_{6}(r)=\pi^{3} r^{6} / 6$. This result was used in an algorithm that measures the density of available points in the vicinity of the point of interest $(\vec{X})$ for hyperspheres of different radii.

If there is a full set of available points within a certain range, then the sample density will be near unity. If, however, there is a scarcity of nearby points, the density at small $r$ values will be much less than 1 . It will then be necessary to determine how the density increases to a maximum at some larger range. As long as the set of sample data is less than complete, this density must fall below unity at some range of $r$ values. It therefore makes sense to choose $\sigma$ as the minimum distance at which the ratio $F(r)=N(r) / V_{6}(r)$ is maximized, where $N(r)$ is the number of sample points within range $r$. That is, we choose $\sigma$ to be the minimum range $r$ at which the density, $F(r)$, is a local maximum. Of course, if we set $r$ large enough, $V_{6}(r)$ will always be much larger than $N(r)$ because the array of computed results will always be finite in nature, whereas $V_{6}(r)$ can increase to infinity. Thus we expect that there will always be some local maximum, but that $F(r)$ may remain relatively close to its maximum value for some range of distances as the array becomes more fully populated with computed results. It therefore makes sense to choose the minimum value at which this maximum is reached so that points at large distances are not overweighted in the least-squares method. 


\section{SWOE field program calculations}

To demonstrate model interoperability between the AIM models and the SWOE thermal model, meteorological information collected during the three SWOE field programs (Grayling I, Yuma, and Grayling II) were used in generating AIM surface solar loading data sets for selected canonical weather conditions. Three cloud scenarios (clear, partly cloudy, and overcast) were chosen for each data site. Hourly surface weather observations were used to determine primary model inputs. In addition, a morning rawinsonde data set was used in estimating cloud layer thickness and base height. We calculated the direct and diffuse components of the solar loading for an 8- by 8-km grid at a $0.25-\mathrm{km}$ spatial resolution and then used a smoothing interpolation method to produce a 0.1-km resolution output grid compatible with the SWOE models.

\section{Semi-empirical surface radiation calculations}

Shapiro $(1972,1982,1987)$ has developed a simple model to determine the direct and diffuse shortwave fluxes at the surface using only standard surface meteorological observations. This model has been used successfully to initialize surface energy budget models. Unlike the AIM model, this model does not produce spatially distributed solar fluxes. For clear skies and overcast conditions, using nondistributed flux values may have little impact on distributed surface energy budget models, provided the spatial domain is not too large. Using nondistributed fluxes for partly cloudy conditions raises some interesting issues.

\section{Model description}

The basic model approach involves dividing the atmosphere into $k$ layers and assuming

$$
R_{k}+T_{k}+A_{k}=1
$$

where $R, T$, and $A$ are the reflectance, transmission, and absorption of layer $k$, respectively. $R, T$, and $A$ have been parameterized in terms of the solar zenith angle $\theta_{o}$ and the state of the atmosphere/clouds using the very extensive SOLMET database (NOAA SOLMET Vol. 2 1979). The general form of the flux equations is

$$
\begin{aligned}
& I_{s \downarrow}^{k}=T_{k} I_{s \downarrow}^{k-1}+R_{k} I_{s \uparrow}^{k} \\
& I_{s \uparrow}^{k}=T_{k+1} I_{s \uparrow}^{k+1}+R_{k+1} I_{s \downarrow}^{k} .
\end{aligned}
$$


Layer $k=0$ is the top of the atmosphere. For operational use, the atmosphere has been divided into three layers consistent with the concept of low, middle, and high clouds, where layer 1 corresponds to the high cloud region, layer 2 to the middle cloud region, layer 3 to the low cloud region, and layer 4 is the ground. The downwelling solar flux at the ground (bottom of layer 3 ) is given as

$$
I_{s \downarrow}=T_{1} T_{2} T_{3} / D_{2}
$$

and

$$
D_{2}=d_{3}\left(d_{1} d_{2}-R_{1} R_{2} T_{2}^{2}\right)-d_{1} R_{2} \alpha_{\text {gnd }} T_{3}^{2}-R_{1} \alpha_{\text {gnd }}\left(T_{2} T_{3}\right)^{2}
$$

and

$$
d_{j}=1-R_{j} R_{j+1} .
$$

$\alpha_{\text {gnd }}$ is the ground albedo. Assuming the total transmission $T_{k}$ can be specified as the sum of the transmission of the direct solar component $T^{d i r}$ and the diffuse solar component $T^{\text {dif }}$.

$$
T_{k}=T_{k}^{d i r}+T_{k}^{d i f} .
$$

The direct solar flux component at the ground is given as

$$
I_{s \downarrow}^{d i r}=T_{1}^{d i r} T_{2}^{d i r} T_{3}^{d i r} I_{o \downarrow}
$$

and the diffuse component as

$$
I_{s \downarrow}^{\text {diff }}=I_{s \downarrow}-I_{s \downarrow}^{\text {dir }} .
$$

$I_{o \downarrow}\left(=1369.3 \mathrm{~W} / \mathrm{m}^{2}\right)$ is the shortwave flux at the top of the atmosphere. To solve the above equations for the downwelling direct and diffuse flux, it is necessary to assume

$$
T_{k}^{\text {dif }}=R_{k}
$$

and therefore $T_{k}^{d i r}$ can be obtained from

$$
T_{k}^{d i r}=T_{k}-T_{k}^{d i f}
$$


Shapiro $(1972,1982,1987)$ parameterized $T_{k}$ and $R_{k}$ in terms of the atmospheric and cloud conditions as follows:

$$
\begin{aligned}
& R_{k}=\varphi_{k} \rho_{k}+\left(1-\varphi_{k}\right) r_{k} \\
& T_{k}=\varphi_{k} \tau_{k}+\left(1-\varphi_{k}\right) t_{k} \\
& \varphi_{k}=W f_{k} .
\end{aligned}
$$

$\rho_{k}$ is the cloud reflectance for cloud layer $k, r_{k}$ the clear sky reflectance for layer $k, \tau_{k}$ the cloud transmission for layer $k, t_{k}$ the clear sky transmission for layer $k$, $f_{k}$ the fractional cloud amount for layer $k$, and $W$ is a cloud weighting factor. $\rho$, $r, t, \tau$, and $W$ are parameterized in terms of the cosine of the solar zenith angle using the SOLMET data set.

$$
\begin{aligned}
& \rho_{k}=a_{k}^{o}+a_{k}^{1} \cos \theta_{o}+a_{k}^{2} \cos ^{2} \theta_{o}+a_{k}^{3} \cos ^{3} \theta_{o} \\
& r_{k}=a_{k}^{o}+a_{k}^{1} \cos \theta_{o}+a_{k}^{2} \cos ^{2} \theta_{o}+a_{k}^{3} \cos ^{3} \theta_{o} \\
& t_{k}=a_{k}^{o}+a_{k}^{1} \cos \theta_{o}+a_{k}^{2} \cos ^{2} \theta_{o}+a_{k}^{3} \cos ^{3} \theta_{o} \\
& \tau_{k}=a_{k}^{o}+a_{k}^{1} \cos \theta_{o}+a_{k}^{2} \cos ^{2} \theta_{o}+a_{k}^{3} \cos ^{3} \theta_{o} .
\end{aligned}
$$

$a_{k^{\prime}}, a a_{k}, b_{\mathrm{k}}$, and $b b_{k}$ are parameterized in terms of the following atmospheric and cloud categories: clear, smoke and haze, thin cirrus and cirrostratus, thick cirrus and cirrostratus, altostratus and altocumulus, and low clouds. The cloud weighting factor $W$ is given as

$$
W=c_{o}+c_{1} \cos \theta_{o}+c_{2} f_{k}+c_{3} f_{k} \cos \theta_{o}+c_{4} \cos ^{2} \theta_{o}+c_{5} f_{k}^{2} .
$$

The $c$ s are parameterized in terms of the following cloud categories: thin cirrus and cirrostratus, thick cirrus and cirrostratus, altostratus and altocumulus, and low clouds. The value of the coefficients can be found in Shapiro (1987). 


\section{Atmospheric transmission model: MODTRAN}

MODTRAN is a MODerate-resolution TRANsmittance (Berk et al. 1989) model for predicting atmospheric radiance and transmittance. Specifically, it calculates atmospheric transmittance, single-scattered solar and lunar radiance, direct solar and lunar irradiance, and multiple-scattered solar and lunar radiance. MODTRAN calculates continuum-type and line (utilizing band models that are a function of pressure, temperature, and line width) molecular absorption, molecular scattering, and aerosol and hydrometer absorption and scattering. The spectral resolution of the model is $2 \mathrm{~cm}^{-1}$ (full width at half-maximum) in averaged steps of $1 \mathrm{~cm}^{-1}$. The spectral fluxes calculated by MODTRAN are integrated over the appropriate spectral interval to generate the broadband solar fluxes. MODTRAN is basically a plane parallel model and cannot compute the fluxes for partly cloudy skies directly. This is achieved by weighting the MODTRAN fluxes for clear and overcast sky conditions with the appropriate cloud amounts.

\section{Measured solar flux values}

The measured fluxes have been obtained from the SWOE data set. Under the SWOE program, field campaigns were conducted at two locations for three separate time periods. The Grayling site chosen to represent the NATO European analog is on one of the tank firing ranges in the Camp Grayling Military Reservation, northeast of Grayling, Michigan. This area is typically rolling hills with a mixture of vegetation types from bare soils to grasses to forests. The underlying soils are predominantly deep sands with little organic material in the near-surface layers. The specific area used in the SWOE Grayling I (September and October 1992) and Grayling II (4 March 1994 to 15 April 1994) field programs is a valley oriented roughly northeast-southwest, characterized by an open, somewhat grasscovered floor. Ridges to the east and west are predominantly covered by mixedgrowth trees (mostly pine and oak). This valley is about $2 \mathrm{~km}$ long by $1 \mathrm{~km}$ wide. Except for very low sun elevation angles, the surrounding ridges do not shadow the pyrheliometer and pyranometer used to collect the solar flux information.

The site at Yuma Proving Ground, Arizona, chosen for SWOE's southwest Asian analog, was characterized as upland Sonoran desert with primary and secondary washes throughout. Soils were varied from highly graded coarse gravel in the wash areas to large expanses of desert pavement on undisturbed ridges. Underlying soils were predominantly mixed gravel and sands over the test area. Vegetation ranged from short grasses to cacti to large palo verde trees. The wash pattern was evident due to the abundance of vegetation in these low areas. At the start of the test, the entire region was extremely wet after several months 
of higher than average rainfall. During the later part of the field program, it was very dry and during the day there was considerable airborne dust. 


\section{THERMAL (SWOETHERM) MODEL}

SWOETHERM is a one-dimensional state-of-the-ground model that is an aggregate of three interactive models. SWOETHERM consists of a soil/snow thermal model, a vegetation (grasses) thermal model, and a canopy (deciduous or coniferous) thermal model. When vegetation or canopy exists the modifications of fluxes due to the interaction of the vegetation or canopy with the underlying soil surface are taken into consideration.

\section{Soil/snow thermal model}

The soil/snow model used in SWOETHERM is SNTHERM (Jordan 1991), developed by Rachel Jordan. SNTHERM is a one-dimensional mass and energy balance model for predicting the temperature profile within strata of snow and soil. It considers the transport of liquid water and water vapor and the phase changes of water as components of the heat balance equation. The impacts of snow accumulation, ablation, densification, and metamorphosis on the snow thermal and optical properties are modeled. The infiltration of water in the snow is modeled assuming gravity flow. When snow is present, the water infiltrating to the snow/soil interface is artificially drained from the system. The snow and soil are divided into horizontally infinite control volumes, and the mass and heat balance equations are applied to each control volume. A spatial discretization scheme similar to a finite-difference method is used in the spatial domain, while a Crank-Nicolson method is used to discretize the time domain. The model uses an adaptive time-step procedure that automatically adjusts the time step (typically between 900 and 5 seconds) to obtain the desired accuracy of the solution to the mass and heat balance equations. The governing equations are linearized, and a tridiagonal-matrix algorithm is used to obtain the desired solution. The required meteorological boundary conditions, which are either user-supplied or generated internally in the model, are air temperature, dew point temperature, wind speed, precipitation, and the solar and downwelling infrared fluxes. The soil and snow layer physical properties required by the model can either be supplied by the user or will default to the hardwired values in the code. The same is true for the profiles of temperature and water in the snow and soil layers.

\section{Vegetation thermal model}

The energy budget of a simple vegetation layer on a soil surface is modeled using a steady-state semi-infinite plane parallel model (Balick et al. 1981), which is described by the foliage emissivity and albedo, a foliage height, the foliage 
fractional coverage and a foliage state parameter. The vegetation consists of a single homogeneous layer that is infinite in both $\mathrm{x}$ and $\mathrm{y}$ directions. The sum of the energy terms, consisting of the absorbed solar and infrared fluxes, the emitted longwave flux, and the sensible and latent heat fluxes, is equal to 0 at each time increment. The solution of the resulting polynomial equation of degree $n$ for the foliage temperature is obtained using a root-finding algorithm or by linearization of the equation. The original model used a root-finding algorithm, but more recently it has been modified to use a linearization procedure. The foliage fractional coverage and the shortwave albedo are a function of the vegetation type (high, medium, or low) and the season (winter, spring, summer, or fall) and are hardwired in the software.

\section{Canopy thermal model}

The canopy model (Smith et al. 1981) is a semi-infinite, steady-state plane parallel energy budget model consisting of three canopy layers, an atmospheric layer above the canopy, and a ground layer below the canopy. The model considers the longwave and shortwave fluxes and the interactions between the various layers and uses a simplified expression for the sensible heat and evapotranspiration flux calculations. The model also incorporates the orientation and distribution of leaves in the canopy layers. Both a longwave and a shortwave transfer matrix are computed for each canopy layer based on a leaf frequency distribution model and the leaf area index. This matrix is used to compute the transfer of longwave and shortwave fluxes within the canopy and the interaction of the canopy-layer-emitted longwave flux. 


\section{$4 \quad$ ANALYSIS}

\section{Instrument variability}

To compare the spatial variations of the measured and AIM-derived solar flux values for Grayling and Yuma, it is necessary to remove, or at least quantify, variations due to inconsistencies between the instruments. The ten Eppley 8-48s and five Eppley PSPs used to obtain the solar flux measurements during the Yuma test were located at a single site, and data were collected under clear-sky conditions. Figure 1 is a plot of the maximum, mean, minimum, and standard deviation for each instrument type for the Yuma measurements. At approximately 0800 and 1730 local, the standard deviation and the measured solar flux values show a relatively large spike. This is an unknown artifact and may be associated with sun glint off part of the instrument. The standard deviation for both instrument types is on the order of 5 to $10 \mathrm{~W} / \mathrm{m}^{2}$, but there is approximately a $70-\mathrm{W} / \mathrm{m}^{2}$ difference between the mean of the two instrument types around solar noon. The maximum difference between the minimum and maximum flux values for both instrument types was approximately $28 \mathrm{~W} / \mathrm{m}^{2}$ and occurred around 1230 local. The percent difference between either the maximum or minimum and the mean between 0900 and 1700 local was less than 3.5\%.

A similar analysis was performed for instrumentation used during the Grayling II experiment. Only a single instrument type was used at the Grayling II field program. The measurements were made using 14 PSPs taking measurements over an approximately 8-hr period. The data prior to 0900 local vary considerably (see Figure 2). Some of this variation may be due to shadowing associated with a low ridgeline to the east of the site. After approximately 1030 local, the standard deviation remains fairly constant, showing only a slight increase with time from about $10 \mathrm{~W} / \mathrm{m}^{2}$ to about $13 \mathrm{~W} / \mathrm{m}^{2}$. The maximum difference between the maximum and minimum flux values was approximately $55 \mathrm{~W} / \mathrm{m}^{2}$ and occurred around local noon. The percent difference between the maximum and the mean or the minimum and the mean after approximately 0930 local was on the order of 5\%. Data measured before 0930 local is unreliable. 


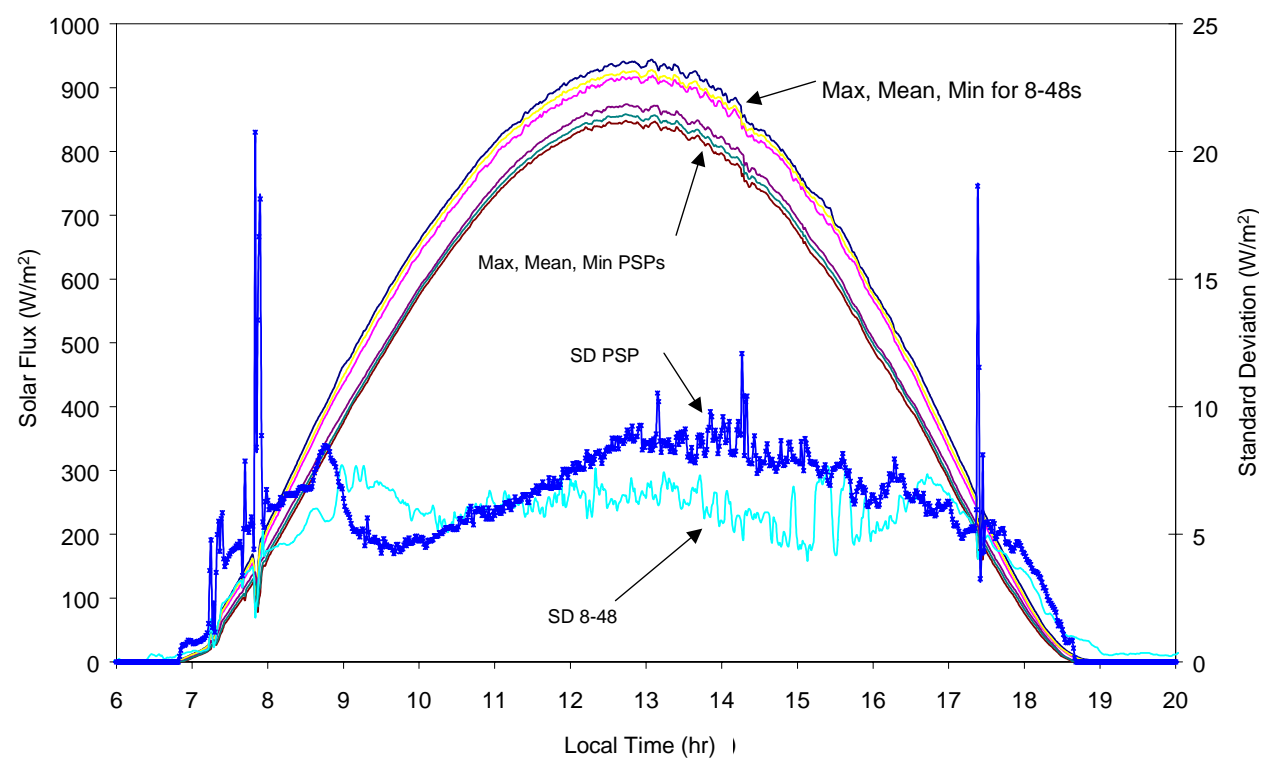

Figure 1. Comparison of co-located 8-48s and PSP for clear-sky conditions at Yuma. Mean, maximum, minimum, and standard deviation are shown for each instrument type.

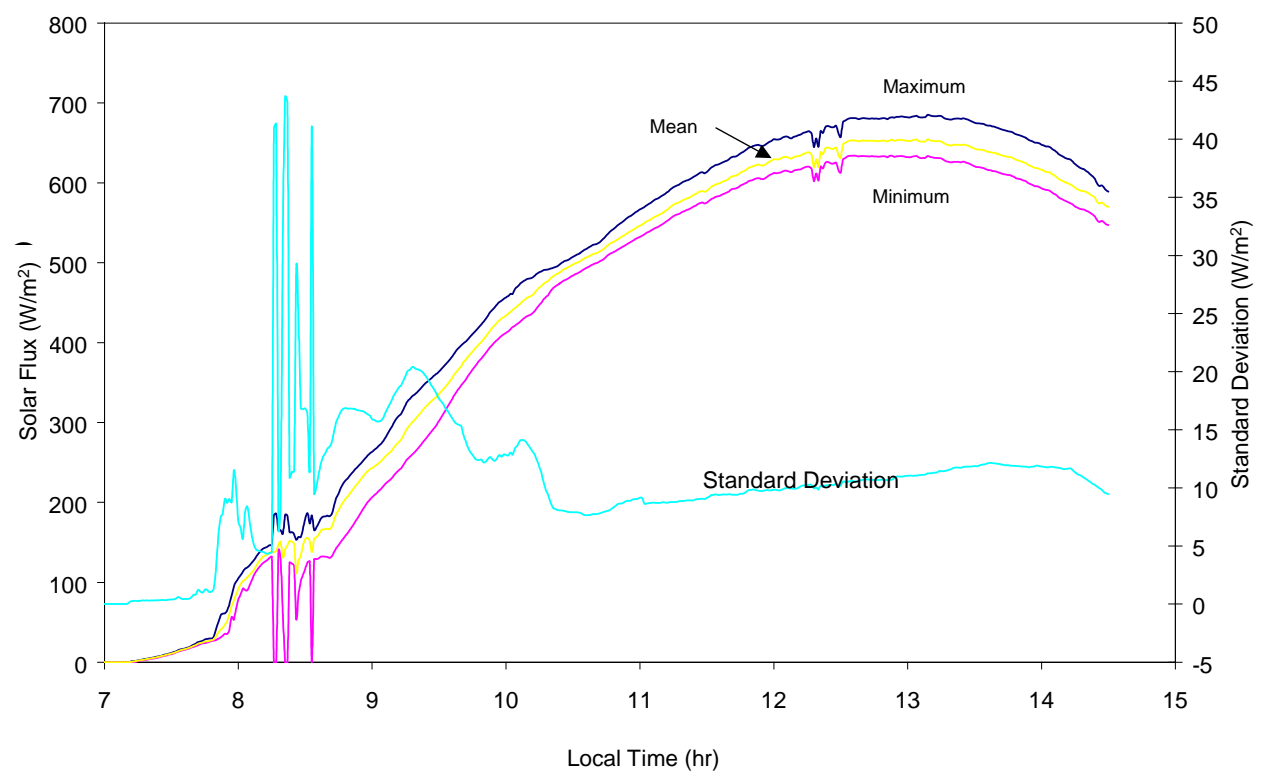

Figure 2. Comparison of 14 PSP for clear-sky conditions during Grayling II. Mean, maximum, minimum, and standard deviation are shown for the 14 instruments. 
For Yuma, the standard deviation and the maximum difference for each set of instrument types are similar. Somewhat unexpected is the rather large mean difference between the two instrument types. This problem will not be encountered for Grayling because only a single instrument type was used. The maximum difference for the Grayling data set is about twice the maximum difference for the Yuma data set. In general, it can be anticipated that 5\%, and possibly as high as $10 \%$, of the variance in the solar flux measurements at any specific time can be attributed to instrument differences. One word of caution: the percent differences and standard deviations are fairly large for solar zenith angles greater than $80^{\circ}$.

\section{Solar flux analysis}

To assess the utility of the three solar flux calculation methods, canonical cloud data sets were selected from each of the three SWOE field programs. Each model produces direct, diffuse, and total solar flux values, but it is primarily the total solar fluxes that are compared. Shapiro's technique generates fluxes based on a semi-empirical technique. The resulting fluxes depend on the cloud conditions, visibility, geographical location, and the time of year and day. MODTRAN is a plane-parallel model that depends on the model atmosphere, visibility and aerosol type, location, and time of year and day. Partly cloudy conditions are taken into consideration by running the model for clear and overcast conditions and weighting the results according to the actual cloud amount. AIM produces spatially distributed solar fluxes for each time step, while the other models and the measured values consist of a single flux value for each time step. The SWOETHERM model was run for bare ground, grass, snow, and deciduous and coniferous canopies using either the measured solar fluxes or the model-calculated solar fluxes. Only the cloud cover varied from model run to model run. All other meteorological conditions were held constant. The model was run for a 24-hr period starting at midnight for each surface condition. The resulting temperature values for each surface condition and cloud cover are compared.

\section{Clear sky flux analysis}

For the clear-sky cases, the AIM solar flux values were constant over the extent of the spatial domain ( $8 \mathrm{~km}$ by $8 \mathrm{~km}$ ). The other models and the measured data also consist of a single solar flux value. 


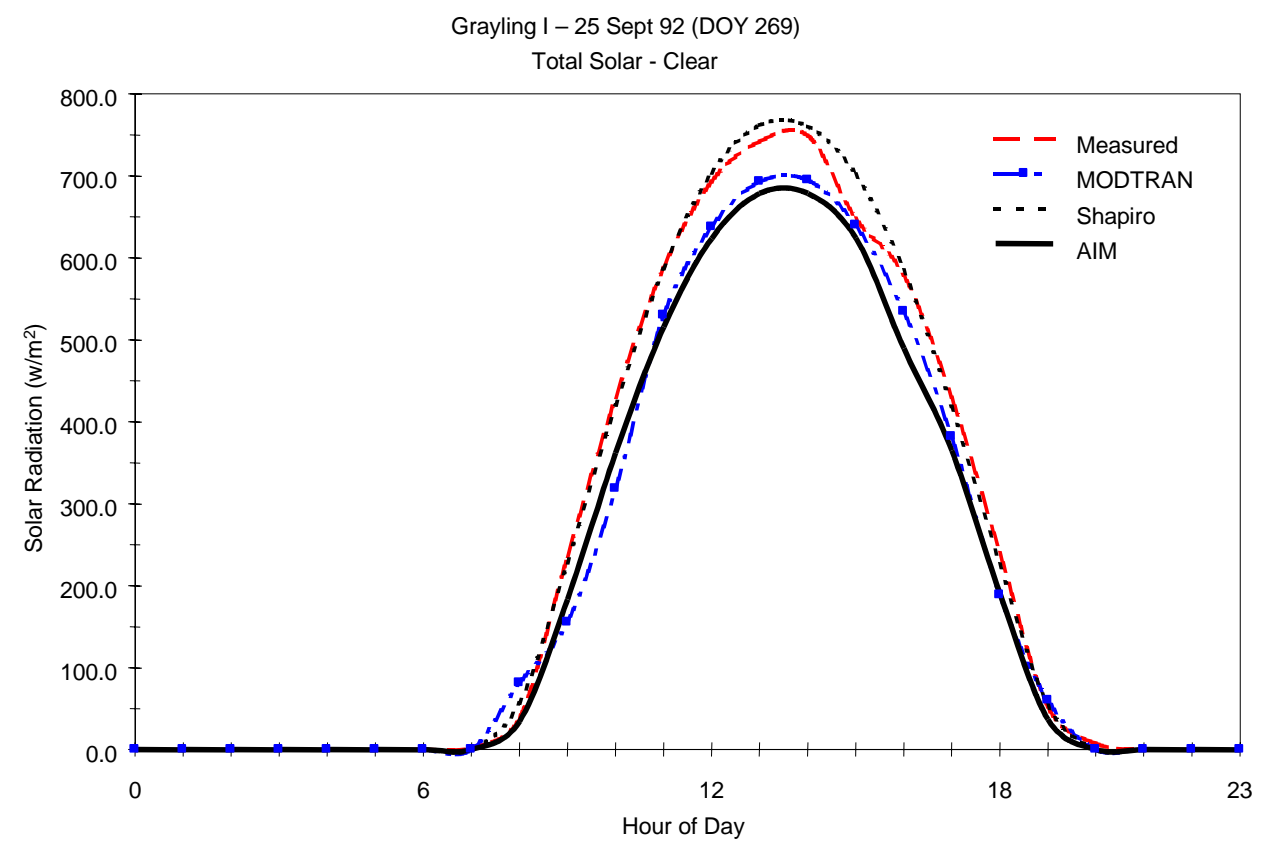

Figure 3. Clear-sky flux, both measured and modeled, based on meteorological conditions from Grayling I field program.

\section{Grayling I: Clear skies}

Figure 3 represents the total solar flux for clear-sky conditions for Grayling I. It is believed that the dip in the measured solar flux values between 1400 and 1600 local is associated with either thin cirrus or a haze layer. The observer reported a haze layer aloft after 1700 local. The maximum difference between the measured total solar flux and the total flux calculated using Shapiro's technique is only $13 \mathrm{~W} / \mathrm{m}^{2}$, and the average difference is only $-2.3 \mathrm{~W} / \mathrm{m}^{2}$.

A comparison for the same time period of two meteorological sites separated by several hundred meters indicates a maximum difference of $25 \mathrm{~W} / \mathrm{m}^{2}$ and an average difference of $10 \mathrm{~W} / \mathrm{m}^{2}$. Both the AIM-calculated total flux and the MODTRAN total flux values are considerably less than the measured values. The maximum difference and average differences between the measured and the AIM-calculated fluxes are $114 \mathrm{~W} / \mathrm{m}^{2}$ and $43 \mathrm{~W} / \mathrm{m}^{2}$, respectively. For the MODTRAN fluxes, the maximum and average differences are $111 \mathrm{~W} / \mathrm{m}^{2}$ and $26 \mathrm{~W} / \mathrm{m}^{2}$, respectively. Although it is not entirely clear why there should be differences of this magnitude, the most likely explanation focuses on how the models handle the aerosol loading in the lower levels of the atmosphere. The Shapiro model does not characterize the surface haze visibility at all, while both the MODTRAN 
and AIM models account for varying haze layer optical depth via standardized vertical profiles based on input surface layer visibility. To explore the relationship between input visibility and solar loading, we ran the AIM model using its standard 2-km-thick haze layer under varying visibility conditions. In Figure 4, as the visibility increases (smaller optical depths), the direct and total solar components increase, while the diffuse component increases until the visibility exceeds $3 \mathrm{~km}$. The decrease in the diffuse component for visibilities greater than 3 $\mathrm{km}$ is due to a decrease in the scattering of the direct component. The solar flux reaching the surface is sensitive to the visibility, especially when the visibility is less than $20 \mathrm{~km}$. During most of the day selected for this comparison, the average visibility was around $28 \mathrm{~km}$.

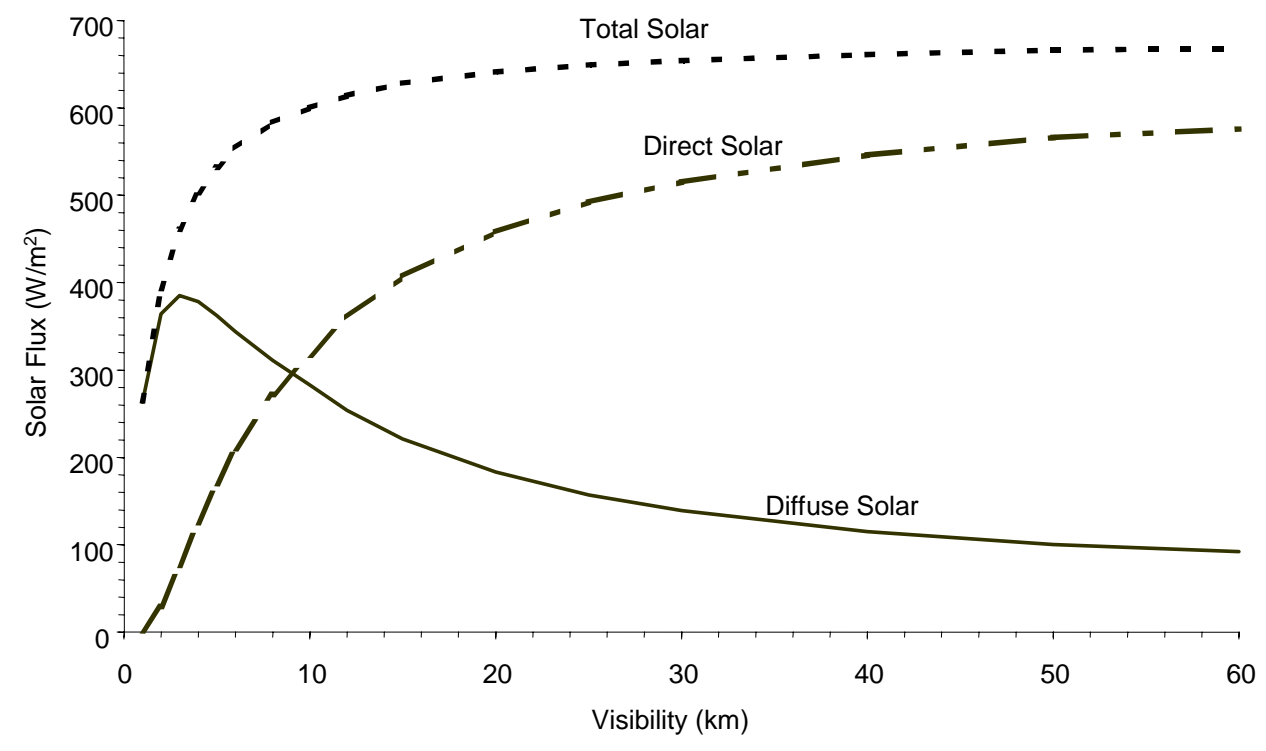

Figure 4. Change in solar flux components as function of visibility as computed using AIM model.

Table 1 summarizes the surface temperature difference between the measured and modeled solar fluxes for different surface materials. The measured minus Shapiro maximum temperature difference (Maxdif) does not exceed $0.5^{\circ}$ for any material type. The average difference (Avgdif) is even less. The relative accuracy of the thermal model is on the order of $1^{\circ} \mathrm{C}$. The measured minus MODTRAN and measured minus AIM maximum temperature difference occurs for bare ground and is on the order of 1 to $2^{\circ} \mathrm{C}$ and the average difference is on the order of a half of a degree. When the surface temperature differences displayed in Table 1 are positive, it indicates the temperature calculated using the model flux is cooler than the value calculated using the measured flux. For example, the 
surface temperature differences associated with the measured minus the AIM fluxes are all positive, indicating the AIM mean flux values are always less than the measured flux values. For this clear-sky case, the Shapiro calculated flux values are the closest to the measured values for Grayling I. The Shapiro model is also the model that has the smallest computational burden. Thus, for clear skies for fairly small spatial domains and for relatively high visibilities, this model offers a rapid method of computing the direct and diffuse solar flux for initializing thermal models.

Table 1. Surface temperature differences $\left({ }^{\circ} \mathrm{C}\right)$ for different surface types and different solar flux initialization schemes for clear-sky conditions for Grayling I.

\begin{tabular}{|c|c|c|c|c|c|c|}
\hline \multirow{3}{*}{ Measured } & \multicolumn{7}{|c|}{ Surface conditions } \\
\cline { 2 - 7 } & & Bare & Snow & Deciduous & Coniferous & Grass \\
\hline \multirow{4}{*}{ MODTRAN } & Maxdif & 1.6 & 1.0 & 0.7 & 0.4 & 0.8 \\
& Mindif & -0.7 & -0.4 & -0.3 & -0.3 & -0.4 \\
& Avgdif & 0.5 & 0.3 & 0.3 & 0.2 & 0.2 \\
\hline \multirow{4}{*}{ Shapiro } & Maxdif & 0.1 & 0.2 & 0.4 & 0.2 & 0.1 \\
& Mindif & -0.5 & -0.3 & -0.3 & -0.1 & -0.2 \\
& Avgdif & -0.1 & -0.1 & 0.1 & 0.1 & 0.0 \\
\hline \multirow{4}{*}{ AIM } & Maxdif & 2.1 & 1.5 & 1.0 & 0.7 & 1.0 \\
& Mindif & 0.0 & 0.0 & 0.0 & 0.0 & 0.1 \\
& Avgdif & 1.1 & 0.7 & 0.5 & 0.3 & 0.4 \\
\hline
\end{tabular}

Yuma: Clear skies

Both the MODTRAN and Shapiro total solar flux values are greater than the measured flux values and the AIM total solar flux values are less than the corresponding measured value. The maximum difference between the measured minus the Shapiro derived flux is $-45 \mathrm{~W} / \mathrm{m}^{2}$, and the average over the daylight period is $-20.5 \mathrm{~W} / \mathrm{m}^{2}$. The maximum difference between the measured minus the MODTRAN flux values is $55.8 \mathrm{~W} / \mathrm{m}^{2}$ and occurs at 0700 local. All other MODTRAN values for the time period are greater than the measured values, with an average difference of $-11.2 \mathrm{~W} / \mathrm{m}^{2}$. The maximum and average difference for the measured minus the AIM total solar flux values is 57.4 and $30.4 \mathrm{~W} / \mathrm{m}^{2}$, respectively. The maximum measured difference for two sites separated by several hundred meters was $28 \mathrm{~W} / \mathrm{m}^{2}$; the average difference between the two sites was $4.0 \mathrm{~W} / \mathrm{m}^{2}$. Again, the difference between measured and modeled fluxes is greater than between two measurement sites. In general, the visibility for the Yuma clear sky day is approximately twice that for the Grayling I clear sky condition. The 
impact of the different solar flux values for different surface types is given in Table 2. As anticipated, the maximum temperature difference occurs for bare ground and is on the order of the relative accuracy of the thermal model.

\begin{tabular}{|c|c|c|c|}
\hline \multirow[b]{2}{*}{ Measured } & \multicolumn{3}{|c|}{ Surface conditions } \\
\hline & & Bare & Grass \\
\hline \multirow[b]{3}{*}{ MODTRAN } & Maxdif & 1.4 & 0.6 \\
\hline & Mindif & -1.1 & -0.3 \\
\hline & Avgdif & -0.1 & 0.0 \\
\hline \multirow[b]{3}{*}{ Shapiro } & Maxdif & 0.0 & 0.0 \\
\hline & Mindif & -1.7 & -0.7 \\
\hline & Avgdif & -0.3 & -0.1 \\
\hline \multirow[b]{3}{*}{ AIM } & Maxdif & 1.6 & 1.2 \\
\hline & Mindif & 0.0 & -0.1 \\
\hline & Avgdif & 0.4 & 0.2 \\
\hline
\end{tabular}

\section{Grayling II: Clear skies}

The maximum differences in the total solar flux for the measured minus MODTRAN, measured minus Shapiro, and measured minus AIM are $48.0 \mathrm{~W} / \mathrm{m}^{2}$, $15.5 \mathrm{~W} / \mathrm{m}^{2}$, and $85.4 \mathrm{~W} / \mathrm{m}^{2}$, while the average differences are $8.4 \mathrm{~W} / \mathrm{m}^{2}, 1.8$ $\mathrm{W} / \mathrm{m}^{2}$, and $26.0 \mathrm{~W} / \mathrm{m}^{2}$, respectively. The average and maximum difference in the total solar flux for two meteorological sites separated by approximately $100 \mathrm{~m}$ for the same time period was $10.0 \mathrm{~W} / \mathrm{m}^{2}$ and $101.0 \mathrm{~W} / \mathrm{m}^{2}$. The maximum difference occurred between 0725 and 0805 local and may be due to shadowing of one of the sites by a low ridgeline to the east. A plot of the calculated SWOETHERM surface temperatures for bare soil using the different flux initialization schemes is given in Figure 5.

Again, the maximum temperature difference and the greatest average difference occur for bare soil (Table 3). In general, the differences are not greater than the relative accuracy of the thermal model. For all clear-sky scenarios, the flux model used to initialize the thermal model does not significantly change the resulting surface temperature. For this relatively small spatial domain, AIM computes a single value for the solar flux for each time step. This would not be the case for relatively large domains where the solar elevation angle would change 
with location. For clear skies and relatively small spatial domains, a simple flux model like Shapiro's has the advantage of being relatively fast and accurate. For larger spatial domains, the issue of changes in the solar zenith angle must be addressed. Even in these cases, a model like Shapiro's could be run for several locations over the domain to model the effects of solar zenith angle variations on the solar flux at the surface.

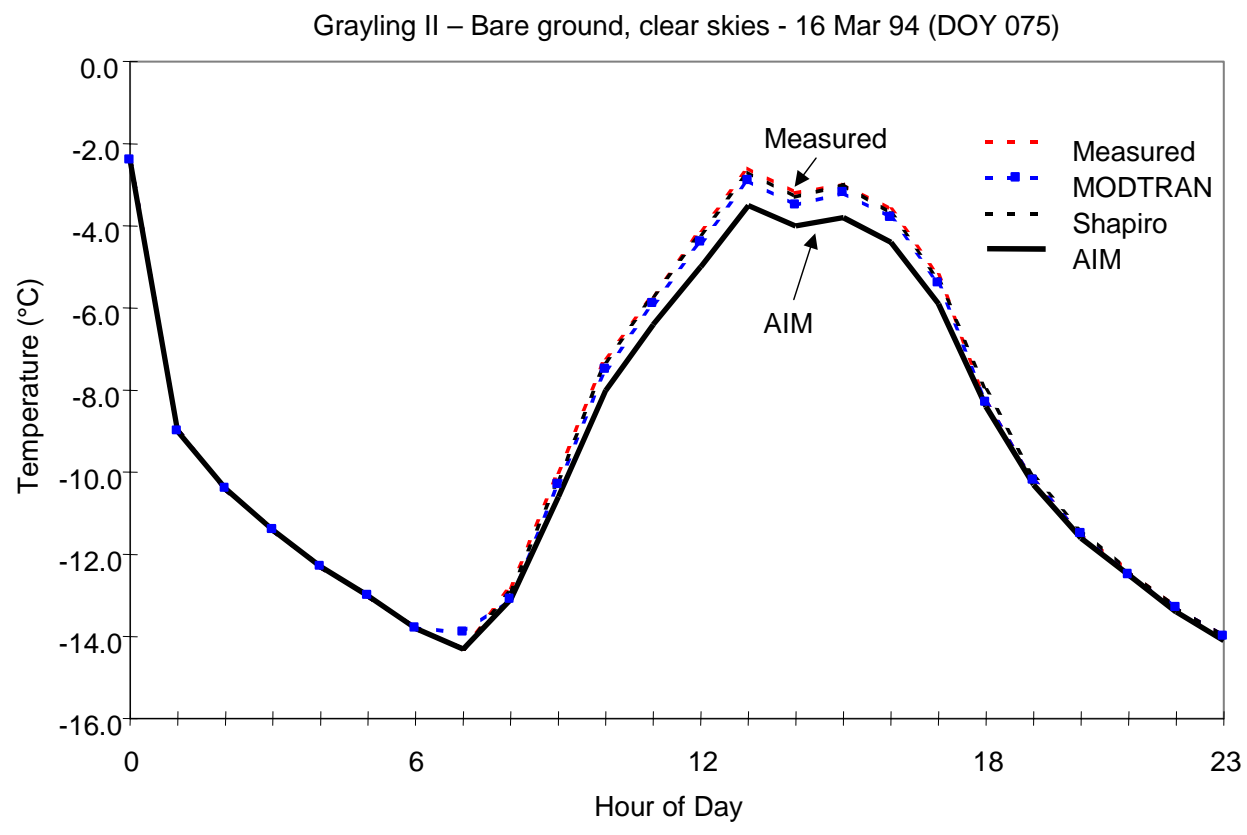

Figure 5. Calculated surface temperature values for bare ground and different solar initialization schemes.

\section{Cloudy sky flux analysis}

To investigate the impact of overcast sky conditions on the calculated solar flux and surface temperatures, days with low overcast cloud conditions were selected when possible. The direct solar flux was generally less than $100 \mathrm{~W} / \mathrm{m}^{2}$ and frequently 0 . For cloudy conditions, the AIM fluxes varied over the spatial extent of the domain. AIM used a $250-\mathrm{m}$ grid resolution that was interpolated to $100-\mathrm{m}$ resolution, resulting in 6400 flux values for the 8- by 8-km domain for each 1-hr time step. Because it would be computationally prohibitive to run SWOETHERM for all 6400 flux values for each hour, the following procedure was used. For each hour, the maximum and minimum flux values were found, and the average of the 6400 values was determined. Three SWOETHERM model 
runs were then made for each hour: one using the maximum value, one using the minimum value, and one using the average value.

Table 3. Surface temperature differences $\left({ }^{\circ} \mathrm{C}\right)$ for different surface types and different solar flux initialization schemes for clear-sky conditions for Grayling II.

\begin{tabular}{|c|c|c|c|c|c|c|}
\hline \multirow{3}{*}{ Measured } & \multicolumn{7}{|c|}{ Surface conditions } \\
\cline { 2 - 7 } & & Bare & Snow & Deciduous & Coniferous & Grass \\
\hline \multirow{3}{*}{ MODTRAN } & Maxdif & 0.3 & 0.3 & 0.2 & 0.1 & 0.2 \\
& Mindif & -0.4 & -0.3 & -0.1 & -0.1 & -0.2 \\
& Avgdif & 0.1 & 0.1 & 0.0 & 0.0 & 0.0 \\
\hline \multirow{4}{*}{ Shapiro } & Maxdif & 0.2 & 0.1 & 0.1 & 0.1 & 0.1 \\
& Mindif & -0.3 & -0.2 & -0.1 & 0.0 & -0.2 \\
& Avgdif & 0.0 & 0.0 & 0.0 & 0.0 & 0.0 \\
\hline \multirow{3}{*}{ AIM } & Maxdif & 0.9 & 0.5 & 0.5 & 0.3 & 0.5 \\
& Mindif & 0.0 & 0.0 & -0.1 & 0.0 & 0.0 \\
& Avgdif & 0.3 & 0.2 & 0.1 & 0.0 & 0.2 \\
\hline
\end{tabular}

Grayling I: Cloudy skies

The distribution of the AIM total solar flux for 1300 local is depicted in Figure 6. Solar flux measurements were made at several sites during the Grayling I field program. All sites are within a circle with a radius of approximately $1 \mathrm{~km}$. Thus it is not possible to determine the spatial distribution of solar flux values from these sites for the 8- by 8-km area. Instead, we have equated the temporal variations of the measured fluxes to the spatial variations as computed with the AIM model. The AIM model in essence represents an instant in time. To generate the temporal variations, we used the 1-min flux values from a single site for a period from 30 minutes before to 30 minutes after the hour. Based on the winds at the cloud height and assuming only cloud advection and no dynamics, during this 1-hr period a cloud would advect approximately $20 \mathrm{~km}$. Using this concept, it would take only 22 minutes for a cloud to be advected across the region. It was felt that using only 22 one-minute observations would not provide a sufficient sample for comparison of the observed data and the AIM-calculated values. Figure 7 is a histogram of the 6400 AIM values produced for conditions at 1300 local, compared to the distribution of the 60 observed values. The AIM model has values in the 500 to $800 \mathrm{~W} / \mathrm{m}^{2}$ range. At 1300 local, the cloud conditions were 0.9 stratocumulus at $300 \mathrm{~m}$ and 0.1 altocumulus at $7000 \mathrm{~m}$. While the AIMcomputed flux values appear higher than the observed conditions, there are 
limitations to the available data set. First, the cloud spatial distribution was generated using CSSM. It appears that CSSM generated areas where there were no clouds or the clouds were created with small optical depths. One explanation for lower optical depths would be the lack of information on the actual thickness of the cloud layers. Estimates of the layer thickness were developed through assessment of the morning rawinsonde flight data, but direct measurements were unavailable. Another explanation is the way cloud fraction information is reported. In the data sets provided, the total fractional cloud cover of all three (low, medium, and high) cloud layers never exceeded unity. Thus, higher cloud layers may have had larger fractional coverage, but were not reported. This would result in an erroneous cloud thickness. Any of these influences would account for the higher flux values, and points to a need for improved reporting requirements during future testing. Yet even for the measured flux values at a single site, the fluxes varied by a factor of two over the 60-min period, indicating that even under overcast conditions the solar flux can vary. These variations may be due to variations in the cloud optical depth.

Total Solar Radiation

Grayling I - 11 Oct 92 (DOY 285) 1300 hr - Cloudy ,

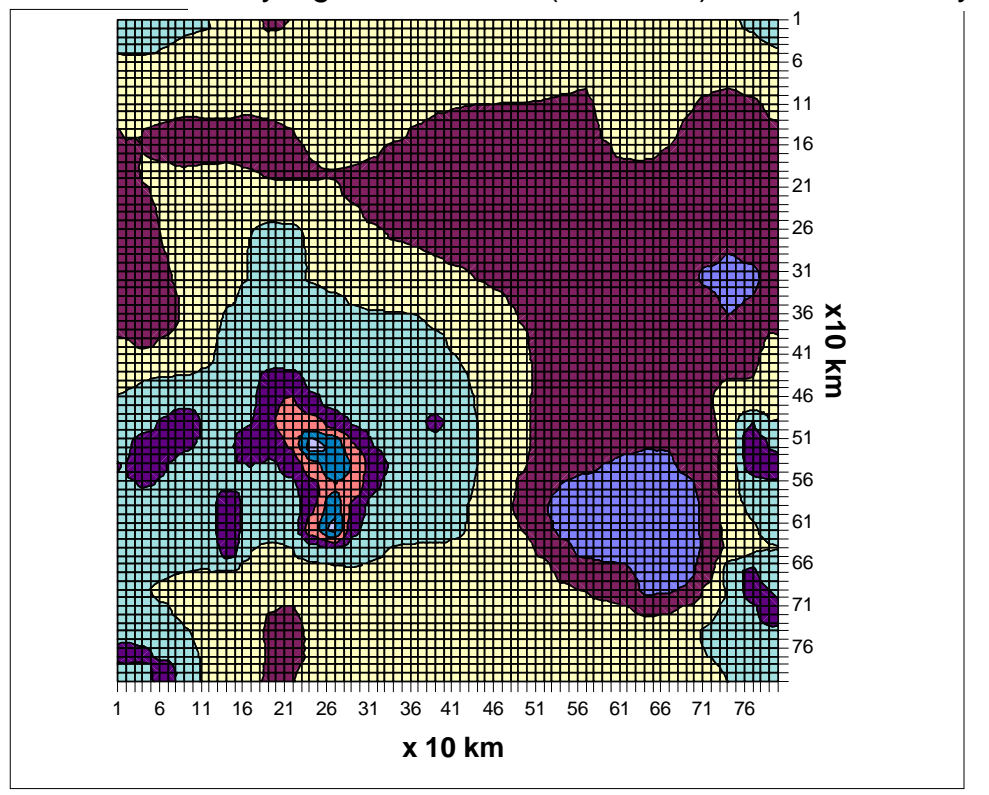

$\square 700.0-800.0$

๘ 600.0-700.0

๘ 500.0-600.0

400.0-500.0

ᄃ 300.0-400.0

ᄃ 200.0-300.0

100.0-200.0

п $0.0-100.0$

Figure 6. Distribution of total solar flux at 1300 local as calculated with AIM model. 


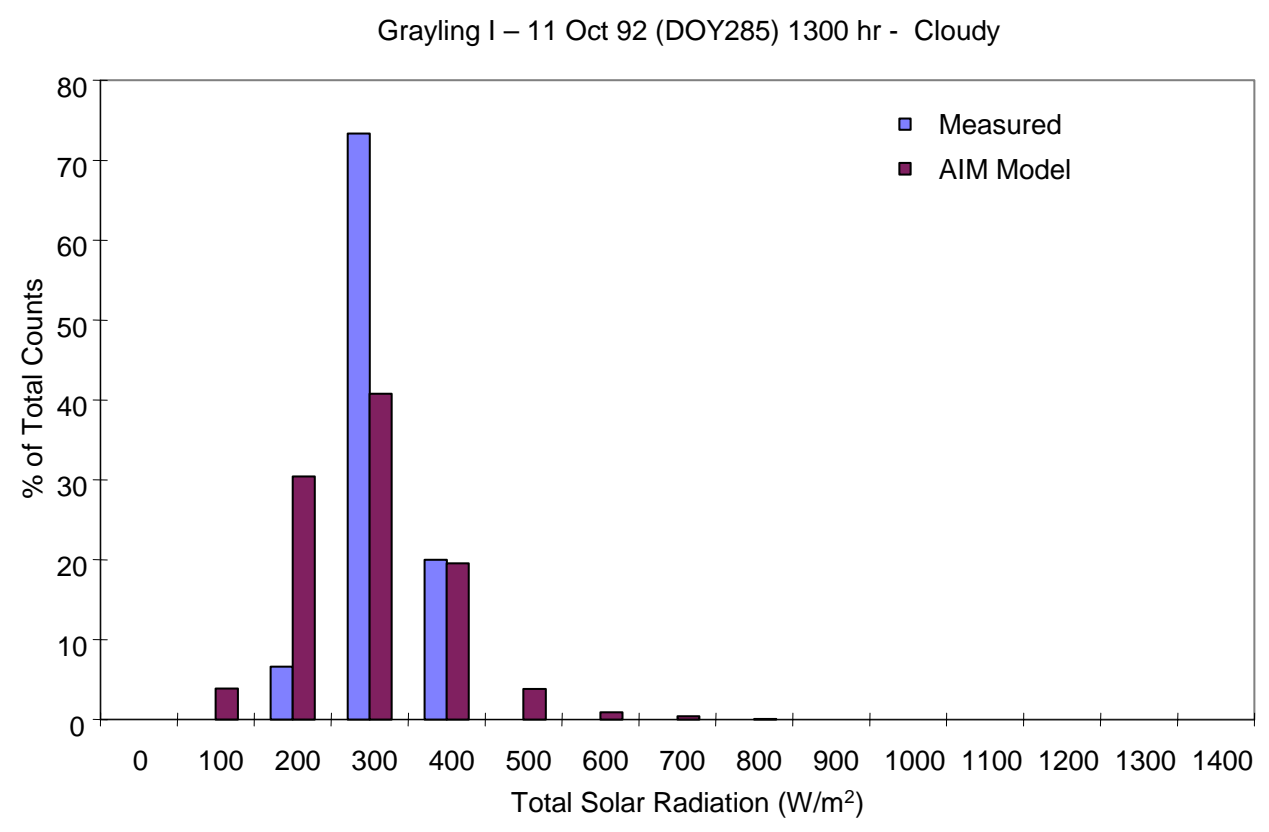

Figure 7. Histogram of AIM fluxes for 1300 local and measured fluxes (see text for details).

The total solar flux values for the different solar radiation initialization schemes are presented in Figure 8. As indicated earlier, at approximately 1300 local the reported sky conditions consisted of nine-tenths low cloud and onetenth middle cloud. Figure 9 presents the resulting surface temperatures from the different initialization schemes for bare soil. The AIM initialization scheme includes the use of the mean, maximum, and minimum flux values for each time period. In general, the maximum difference in the surface temperature for the MODTRAN, Shapiro, and AIM average value initialization scheme is less than $2^{\circ}$. This is not the case for the initializations using the AIM maximum and minimum fluxes. The maximum values result in a surface temperature difference that is on the order of $4^{\circ}$ warmer while the minimum is approximately $4^{\circ}$ cooler than the temperatures computed using the measured flux values. At 1300 local, the difference in the calculated bare soil surface temperature using the maximum and minimum flux is approximately $8^{\circ}$. This represents the extreme since the minimum and maximum flux values are used for the entire period. That is, since the maximum and minimum are used for the entire period leading up to 1300 local there will be an accumulative effect. In reality, a single location in the simulation area would experience a range of flux values as cloud conditions over the location change. We would expect the surface temperature to vary between the upper and lower limits given by the upper and lower curves in Figure 9. The impact of 
the different initialization schemes is summarized in Table 4. Negative values imply the surface temperature computed using the measured flux values. Maxdif, Mindif, and Avgdif are the maximum and minimum temperature difference and the average of the differences for the period when solar fluxes are not zero (daylight). As anticipated, the greatest differences occur when we initialize the model using either the AIM maximum or minimum flux values. In addition, the greatest differences occur for bare surface conditions.

Grayling I - 11 Oct 92 (DOY 285)

Total Solar - Cloudy

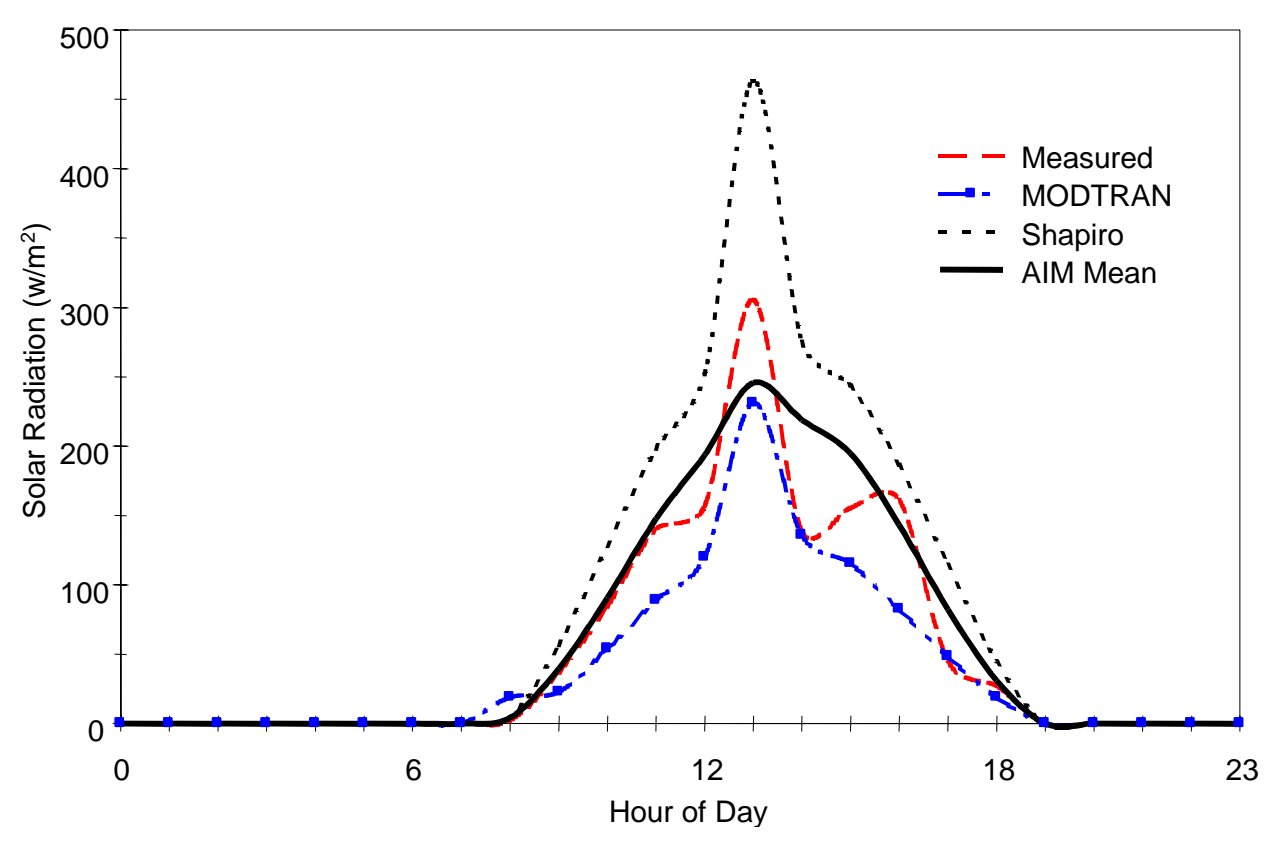

Figure 8. Total solar fluxes for overcast conditions.

With regard to these computations, several items should be noted. First, the AIM average calculations show an average difference that is consistently closer to the measured conditions (within approximately $0.1^{\circ}$ ) than either the MODTRAN or Shapiro methods. The AIM maximum and minimum cases, on the other hand, tend to bracket the observed conditions equally on the high and low side. By comparison, the Shapiro predictions consistently produce predicted temperatures above those predicted using the measured fluxes. The advantage of the Shapiro model is that it requires fewer input parameters and is computationally fast. The MODTRAN estimates are consistently low, probably due to the inability of MODTRAN to model clouds with varying optical depths, as compared to both the Shapiro and AIM methods. 
Grayling - Bare ground, cloudy skies - 11 Oct 92 (DOY 285)

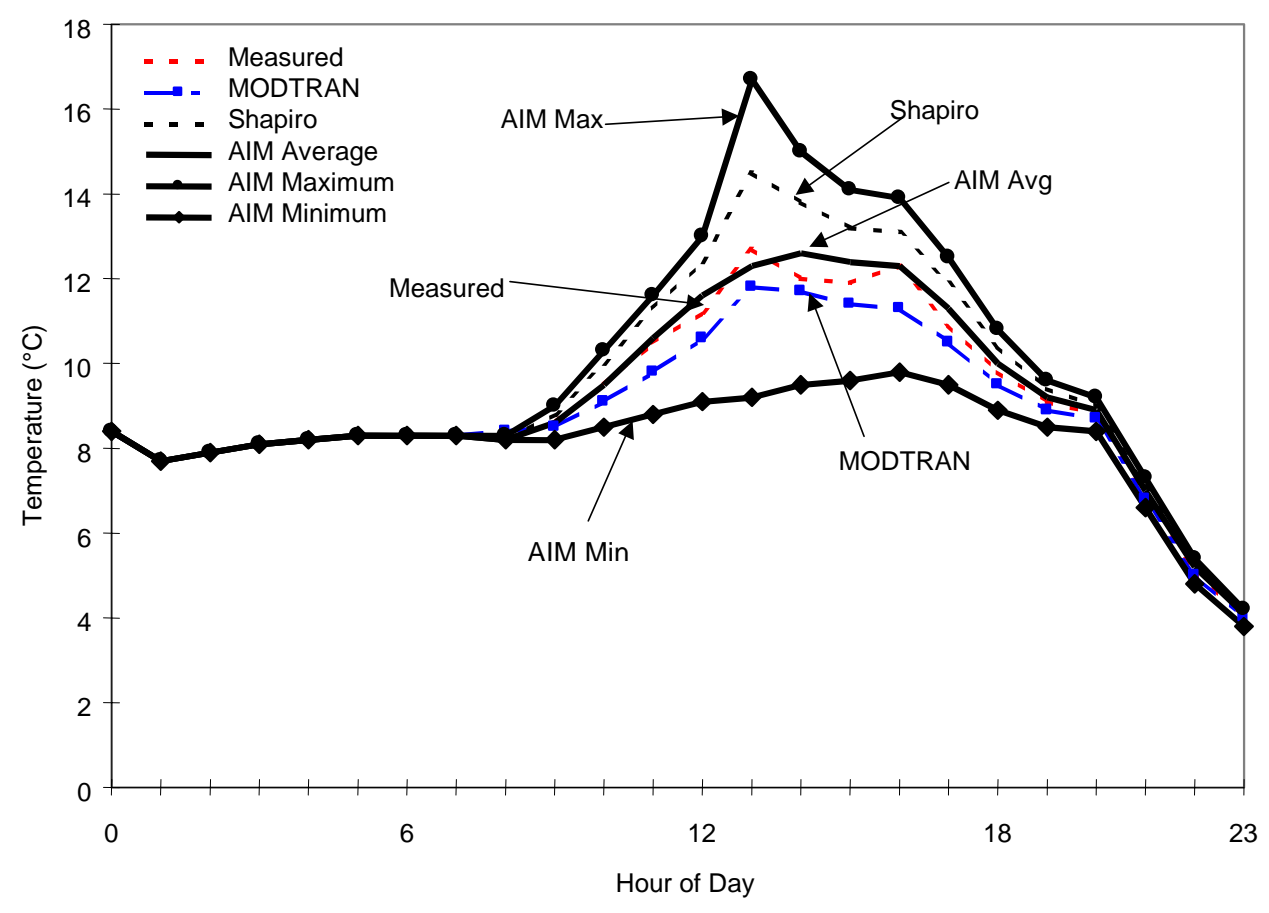

Figure 9. Surface temperature for bare soil calculated using different initialization schemes.

\section{Yuma: Cloudy skies}

During the day the cloud conditions varied from a solid low overcast with rain and drizzle to scattered low clouds with overcast to broken mid-level cloud cover. Comparing the flux values (Figure 10), it appears that the calculated AIM average flux value is not consistent with the peak associated with the measured value at 1000 local. This is somewhat misleading, however, since the variable plotted is the average flux of all 6400 values over the 8- by 8-km region. In general, we are comparing point measurements and point model-generated fluxes (MODTRAN and Shapiro) with spatially distributed model-generated flux values at a single instant of time. Figure 11 is a plot of the maximum flux values for each hour as computed by the AIM model. At 1000 local, it can be seen that there is a spike in the maximum value. 


\begin{tabular}{|c|c|c|c|c|c|c|}
\hline \multirow[b]{2}{*}{ Measured } & \multicolumn{6}{|c|}{ Surface conditions } \\
\hline & & Bare & Snow & Deciduous & Coniferous & Grass \\
\hline \multirow[b]{3}{*}{ MODTRAN } & Maxdif & 1.0 & 0.7 & 0.6 & 0.5 & 0.4 \\
\hline & Mindif & -0.2 & -0.2 & -0.2 & -0.1 & -0.1 \\
\hline & Avgdif & 0.4 & 0.3 & 0.2 & 0.1 & 0.2 \\
\hline \multirow[b]{3}{*}{ Shapiro } & Maxdif & 0.0 & 0.0 & 0.0 & 0.0 & 0.0 \\
\hline & Mindif & -1.8 & -1.3 & -1.1 & -0.7 & -0.7 \\
\hline & Avgdif & -0.8 & -0.5 & -0.5 & -0.3 & -0.3 \\
\hline \multirow{3}{*}{$\begin{array}{c}\text { AlM } \\
\text { Average }\end{array}$} & Maxdif & 0.4 & 0.4 & 0.2 & 0.2 & 0.2 \\
\hline & Mindif & -0.6 & -0.5 & -0.3 & -0.3 & -0.3 \\
\hline & Avgdif & -0.1 & -0.1 & -0.1 & -0.1 & 0.0 \\
\hline \multirow{3}{*}{$\begin{array}{c}\text { AlM } \\
\text { Maximum } \\
\end{array}$} & Maxdif & 0.0 & 0.0 & 0.0 & 0.0 & 0.0 \\
\hline & Mindif & -4.0 & -2.9 & -2.2 & -1.3 & -1.5 \\
\hline & Avgdif & -1.3 & -0.9 & -0.8 & -0.5 & -0.5 \\
\hline \multirow{3}{*}{$\begin{array}{c}\text { AlM } \\
\text { Minimum }\end{array}$} & Maxdif & 3.5 & 2.7 & 1.9 & 1.2 & 1.3 \\
\hline & Mindif & 0.0 & 0.0 & 0.0 & 0.0 & 0.0 \\
\hline & Avgdif & 1.4 & 1.1 & 0.8 & 0.5 & 0.5 \\
\hline
\end{tabular}

Because the cloud amount at this time is 0.8 low cloud and 0.2 middle cloud, only a few of the 6400 pixels have high flux values. Thus, the computed average of the 6400 values tends to wash out the peak at this time. The measured value at 1000 local was $333 \mathrm{~W} / \mathrm{m}^{2}$, and at 1009 it was only $96 \mathrm{~W} / \mathrm{m}^{2}$. Based on the winds at the cloud level, in 9 minutes the clouds could have advected approximately 4 $\mathrm{km}$, a distance significantly larger than the standard gap distance between clouds produced in the AIM domain. This is a limitation of nondistributed flux models or point measurements: we cannot depict the spatial and temporal variability that can occur over these small scales. In this case, it just happens that at 1000 local the lack of cloud cover over the site resulted in fairly high flux values. Another problem that comes into play is, how do models stack clouds vertically? Two approaches are random overlap and maximum overlap. Stochastic models like CSSM (the model used in this work) use a random overlap approach. But is nature random? If there are dynamic processes involved, it is likely that the clouds will be correlated from level to level. Based on the AIM maximum and minimum flux initialization schemes, the potential range of temperatures of the AIM domain is $5^{\circ} \mathrm{C}$ (Figure 12) even though sky conditions are cloudy. Even the range of temperatures computed using MODTRAN, average AIM values over the domain, Shapiro's technique, and the measured is on the order of several degrees. 
These differences reflect the difference in the flux values, which are due in part to how clouds are handled in each scheme. During the period from 1000 to 1500 local, all schemes predict flux values that are greater than the measured values. Even the AIM minimum values are greater than the measured values. This is not surprising, considering the cloud conditions. The cloud types were mainly cumulus and had very dark bases indicating fairly large optical depths. As pointed out earlier, information on the vertical extent of the clouds was not available. Even if this information were available, only the AIM model uses cloud thickness information in the calculation of the solar flux values. For the version of MODTRAN used in this study there are no provisions for using cloud thickness information. Since the coefficients used in the Shapiro model are based on measurements, cloud thickness indirectly plays a role in the calculation of the solar flux values.

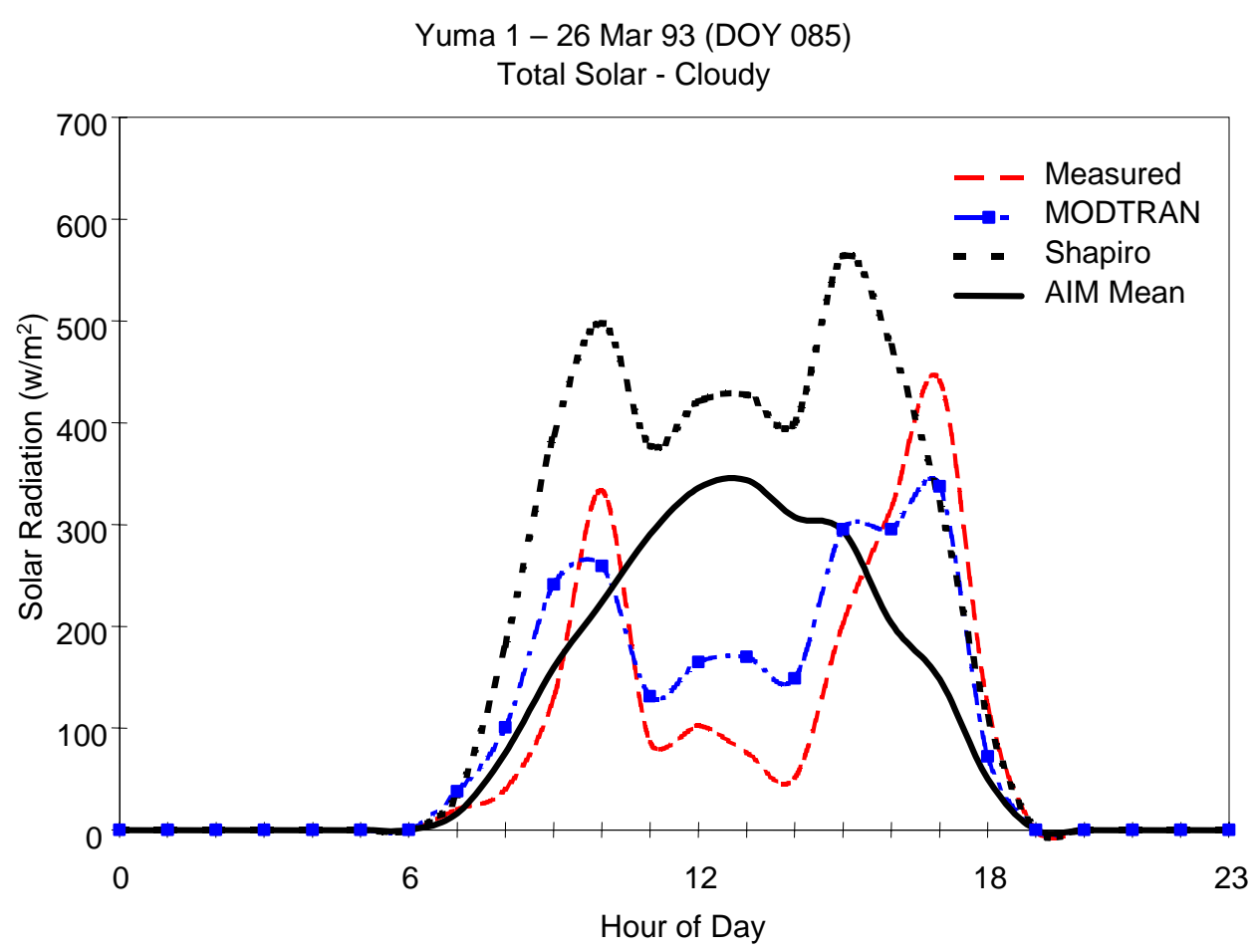

Figure 10. Comparison of different model computed flux and measured flux for cloudy conditions for Yuma.

The impact of variations in the cloud conditions is reflected in the summary statistics presented in Table 5. 
Yuma - Cloudy 26 Mar 93 (DOY 085)

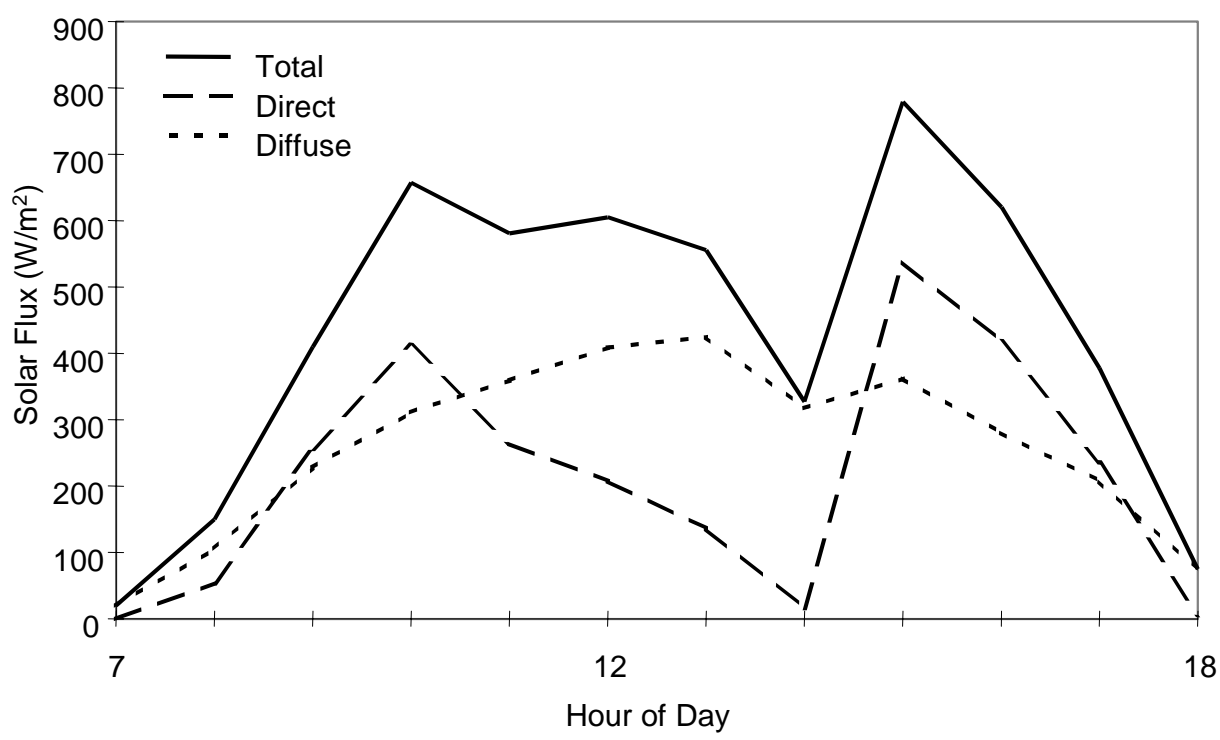

Figure 11. AIM maximum fluxes for Yuma cloudy skies day.

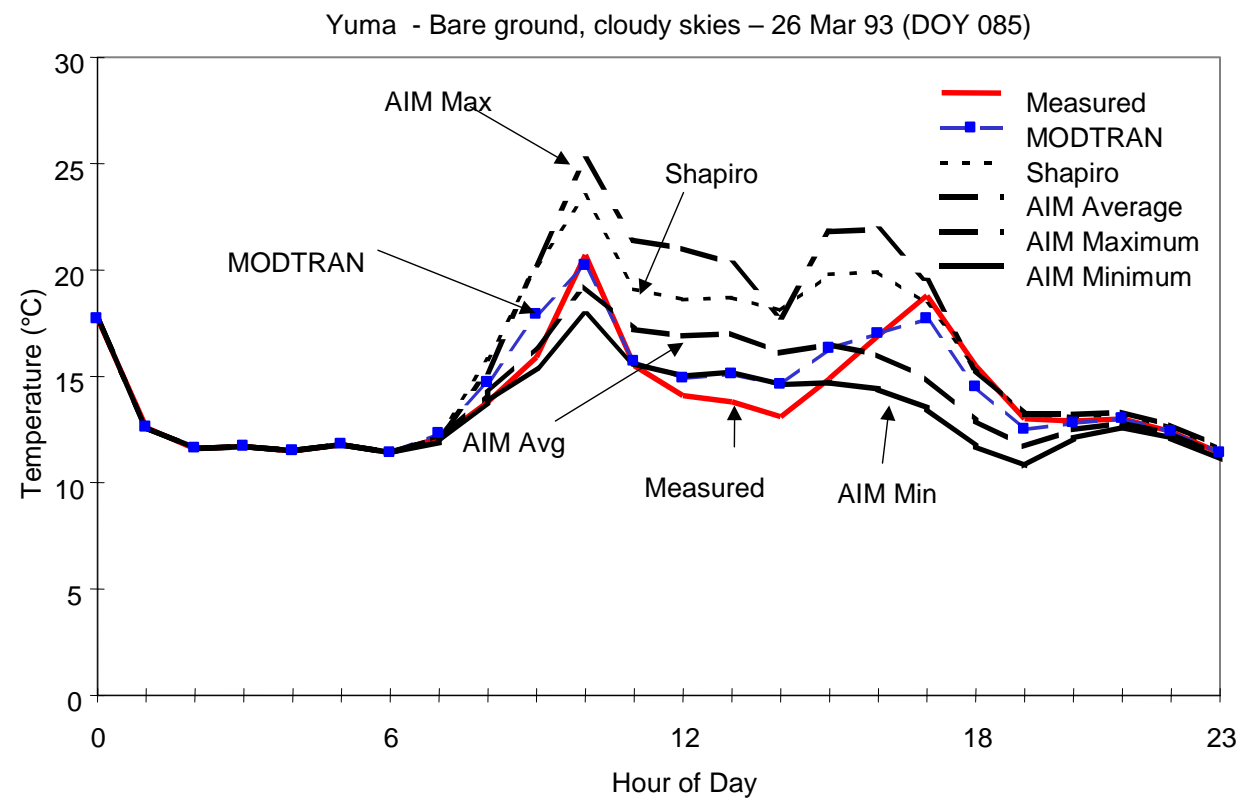

Figure 12. Surface temperature for bare ground using different initialization schemes. 


\begin{tabular}{|c|c|c|c|}
\hline \multirow[b]{2}{*}{ Measured } & \multicolumn{3}{|c|}{ Surface conditions } \\
\hline & & Bare & Grass \\
\hline \multirow[b]{3}{*}{ MODTRAN } & Maxdif & 1.1 & 0.5 \\
\hline & Mindif & -2.0 & -0.6 \\
\hline & Avgdif & -0.2 & -0.1 \\
\hline \multirow[b]{3}{*}{ Shapiro } & Maxdif & 0.4 & 0.4 \\
\hline & Mindif & -5.0 & -1.6 \\
\hline & Avgdif & -1.5 & -0.4 \\
\hline \multirow{3}{*}{$\begin{array}{c}\text { AIM } \\
\text { Average }\end{array}$} & Maxdif & 3.9 & 1.5 \\
\hline & Mindif & -3.2 & -1.7 \\
\hline & Avgdif & -0.1 & 0.0 \\
\hline \multirow{3}{*}{$\begin{array}{c}\text { AIM } \\
\text { Maximum }\end{array}$} & Maxdif & 0.2 & 0.2 \\
\hline & Mindif & -6.9 & -2.4 \\
\hline & Avgdif & -2.0 & -0.5 \\
\hline \multirow{3}{*}{$\begin{array}{c}\text { AIM } \\
\text { Minimum }\end{array}$} & Maxdif & 5.3 & 1.9 \\
\hline & Mindif & -1.5 & -0.5 \\
\hline & Avgdif & 0.6 & 0.2 \\
\hline
\end{tabular}

\section{Grayling II: Cloudy skies}

For the day selected as representing cloudy conditions during the Grayling II experiment, low overcast cloud conditions persisted throughout the entire daylight period. In fact, the measured direct solar flux was essentially zero for all time periods except around local noon. Thus, the total solar loading consisted only of the diffuse component. MODTRAN and the semi-empirical model (Shapiro's model) indicated the direct component was zero for all time periods. A plot of total solar flux values for the different schemes is given in Figure 13.

All models tend to overpredict the value of the total solar flux relative to the measured. As indicated, the total solar flux is essentially the diffuse flux. Because of the low overcast sky conditions we have no information on cloud amounts above the overcast. If upper cloud layers (middle and/or high clouds) were present, or the cloud layer was optically thicker than normal for this type cloud, the optical depth would increase, and the corresponding diffuse solar component may decrease if the total optical depth is greater than approximately 3. This would bring the model values more in line with the measured values. The spike in the measured total solar flux around 1300 local is due to a direct 
component and a significant increase in the diffuse component (see Figure 14). Recalling Figure 4, we see the diffuse component can increase as the optical depth increases up to a certain point and then decreases as the optical depth continues to increase. In addition, the diffuse component can also increase due to an increase in the scattering from the sides of the clouds. Only the AIM model predicted a direct component during this time period. A histogram of the 6400 AIM values and 50 measured values from five measurement sites for a period of 5 minutes before and after 1300 local are presented in Figure 15. The measurement sites are within a circle with a radius on the order of $1 \mathrm{~km}$. Even over this small spatial domain for this relatively short period, there is variability in the flux values, as indicated by the histogram of the measured values in Figure 15. The impact of the different initialization schemes on the surface temperature for bare ground is given in Figure 16. As anticipated, the greatest range of temperature differences occurs around solar noon when SWOETHERM is initialized using the AIM maximum and minimum flux values.

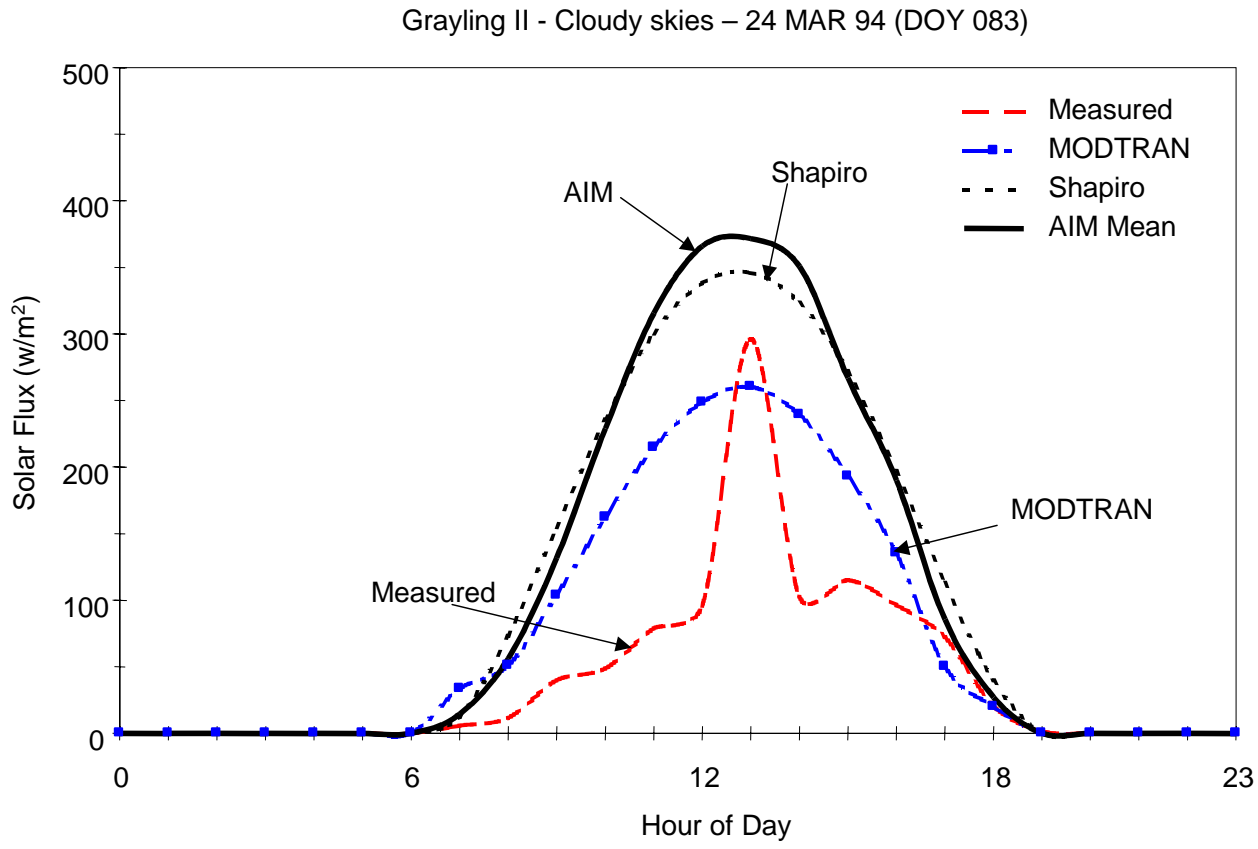

Figure 13. Total solar flux values for overcast low cloud conditions at Grayling II field site.

The range of temperatures for the other surface types is not as great as it is for bare ground. A summary of the results for the different initialization schemes and surface material types is given in Table 6. 
Grayling II - 24 Mar 94 (DOY 083)

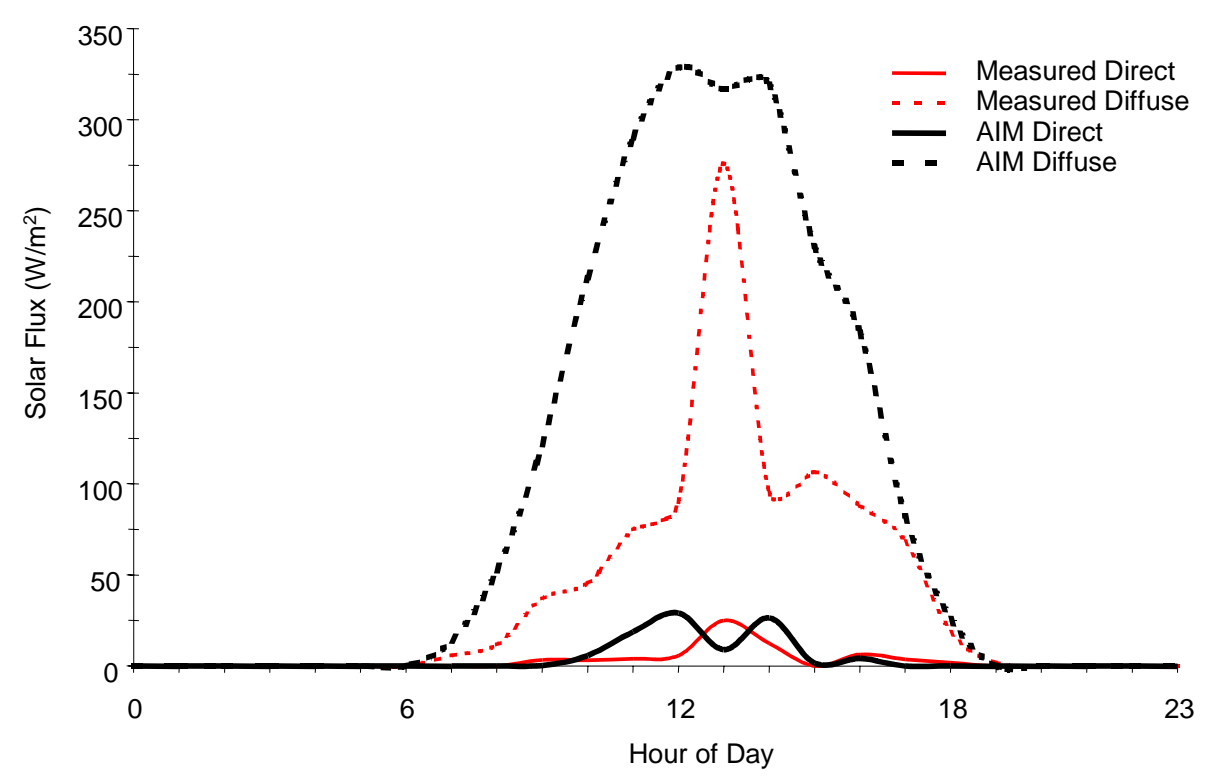

Figure 14. Comparison of direct and diffuse measured and AIM-generated flux values.

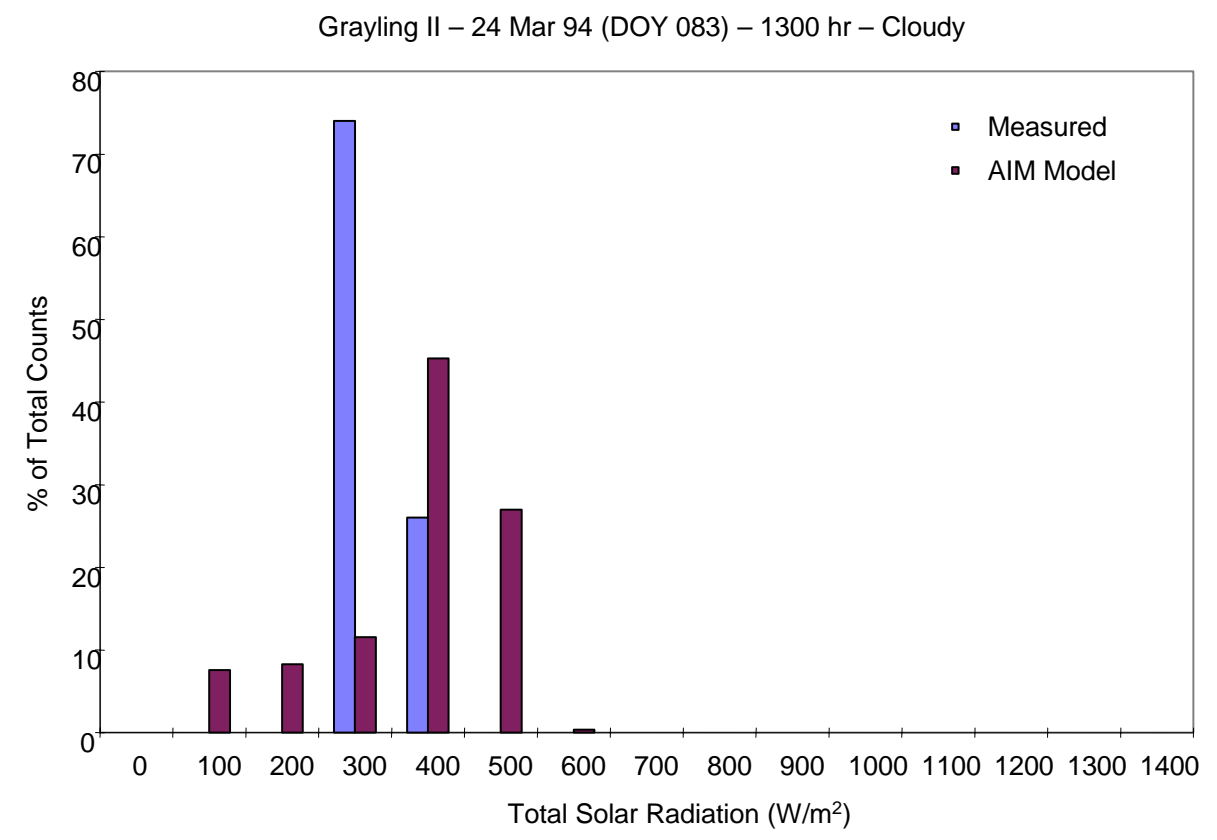

Figure 15. Histogram of total solar flux for 6400 AIM values and 50 measured values. 
Grayling II - Bare ground, cloudy skies - 24 Mar 94 (DOY 083)

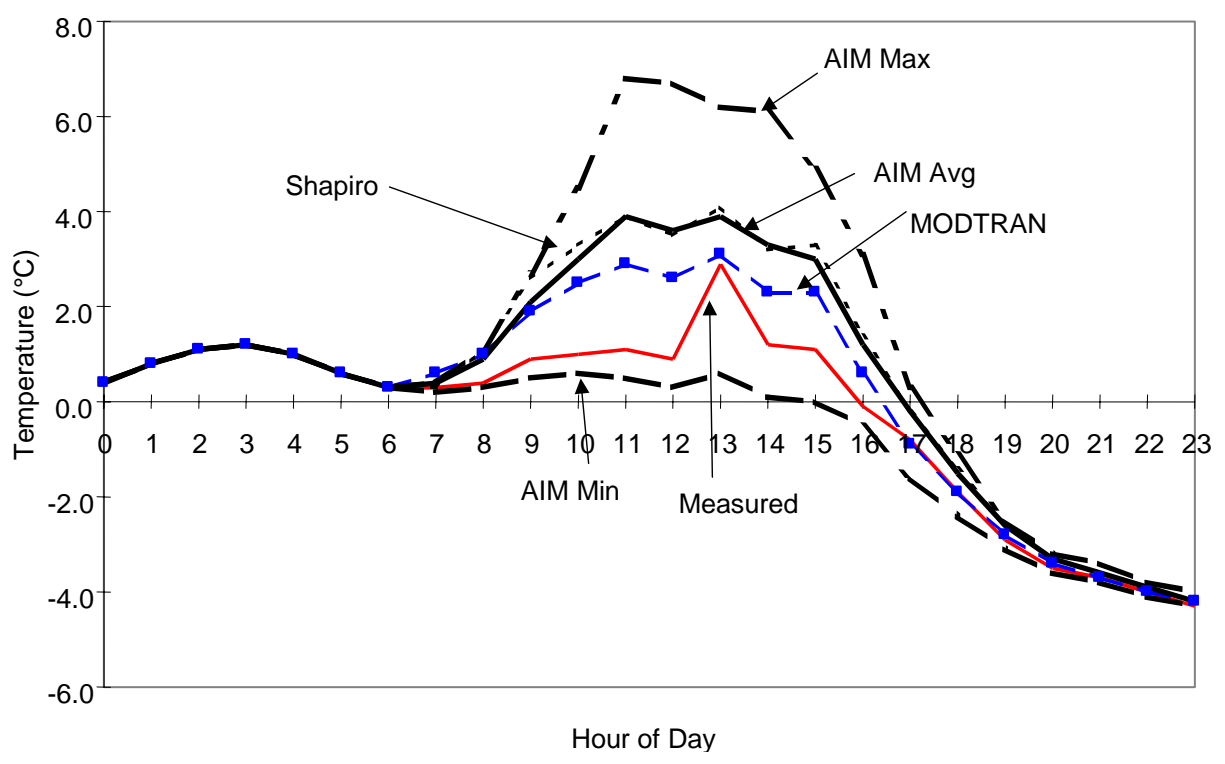

Figure 16. Impact of different flux initialization schemes on bare-ground surface temperature for Grayling II.

\begin{tabular}{|c|c|c|c|c|c|c|}
\hline \multirow[b]{2}{*}{ Measured } & \multicolumn{6}{|c|}{ Surface conditions } \\
\hline & & Bare & Snow & Deciduous & Coniferous & Grass \\
\hline \multirow[b]{3}{*}{ MODTRAN } & Maxdif & 0.1 & 0.0 & 0.0 & 0.0 & 0.0 \\
\hline & Mindif & -1.8 & -0.4 & -0.9 & -0.7 & -0.6 \\
\hline & Avgdif & 0.4 & -0.1 & -0.2 & -0.2 & -0.1 \\
\hline \multirow[b]{3}{*}{ Shapiro } & Maxdif & 0.0 & 0.0 & 0.0 & 0.0 & 0.0 \\
\hline & Mindif & -2.8 & -0.5 & -1.5 & -0.9 & -1.0 \\
\hline & Avgdif & -0.8 & -0.2 & -0.4 & -0.2 & -0.3 \\
\hline \multirow{3}{*}{$\begin{array}{c}\text { AlM } \\
\text { Average } \\
\end{array}$} & Maxdif & 0.0 & 0.0 & 0.0 & 0.0 & 0.0 \\
\hline & Mindif & -2.8 & -0.5 & -1.5 & -0.9 & -1.0 \\
\hline & Avgdif & -0.7 & -0.1 & -0.3 & -0.2 & -0.2 \\
\hline \multirow{3}{*}{$\begin{array}{c}\text { AlM } \\
\text { Maximum } \\
\end{array}$} & Maxdif & 0.0 & 0.0 & 0.0 & 0.0 & 0.0 \\
\hline & Mindif & -5.8 & -1.9 & -3.0 & -1.8 & -2.0 \\
\hline & Avgdif & -1.5 & -0.3 & -0.7 & -0.4 & -0.5 \\
\hline \multirow{3}{*}{$\begin{array}{c}\text { AIM } \\
\text { Minimum }\end{array}$} & Maxdif & 2.3 & 0.8 & 1.2 & 0.7 & 0.6 \\
\hline & Mindif & 0.0 & -0.1 & 0.0 & 0.0 & 0.0 \\
\hline & Avgdif & 0.4 & 0.1 & 0.2 & 0.1 & 0.1 \\
\hline
\end{tabular}


In almost all cases, the differences between the temperature computed using the measured flux values and either MODTRAN, Shapiro's technique, or the average AIM flux values are negative, implying the temperatures computed using the model flux values are warmer than the corresponding temperatures computed using the measured flux values. In general, the model flux values are greater than the measured values. This highlights the importance of using models that account for the differences in the cloud optical depth or cloud vertical extent.

Relying on surface observations to characterize the cloud conditions has a number of limitations. First, we noted cases where upper-level cloud layers may have been present but were obscured from the view of the observers. Second, even when upper levels are visible through gaps in the lower cloud layers, it may not be possible to accurately determine the fractional cover of these upper layers. Finally, cloud optical depth or cloud vertical extent is not readily available from surface observations. Given these data gaps, other methods need to be employed to improve the accuracy of the cloud data. Methods might include improved, or automated, analysis of the rawinsonde data to determine the presence of cloud layers, use of satellite observations to determine the nature of upper level clouds, and/or coupling of observer information with outputs from numerical weather predictions. In this latter case, models for determining positions of cloud layers and fractional coverage are available, such as the Atmospheric Sounding Program (ASP) (Passner 1998), which is a numerical weather post-processing algorithm run as part of the Army's Integrated Meteorological System (IMETS). This model has approximately a 70\% skill level in assessing cloud amount and level height for large cloud systems; that is, clouds not involving vertical dynamics like cumulus cloud development. Given data integration of this sort, it would be possible to gain a better estimate of cloud conditions. Looking at the problem from another perspective, if surface solar loading information were available, model outputs could be used to infer cloud conditions above an obscuring lower cloud level.

\section{Partly cloudy sky flux analysis}

As indicated in the introduction, distributed models potentially require distributed solar flux values, especially for partly cloudy conditions. In partly cloudy conditions, low clouds cast hard shadows and it is possible to transition from full solar loading conditions to diffuse solar loading over small spatial extents and short periods of time. The solar loading at any given point is modulated by the advection of clouds and, to some extent, the evolution of the cloud geometry. In dry environments such as Yuma, there can be dramatic changes in surface temperature over short periods of time or over small spatial extents. 
Figure 17 clearly illustrates the impact of temporal variations in the measured total solar flux on the measured surface temperature.

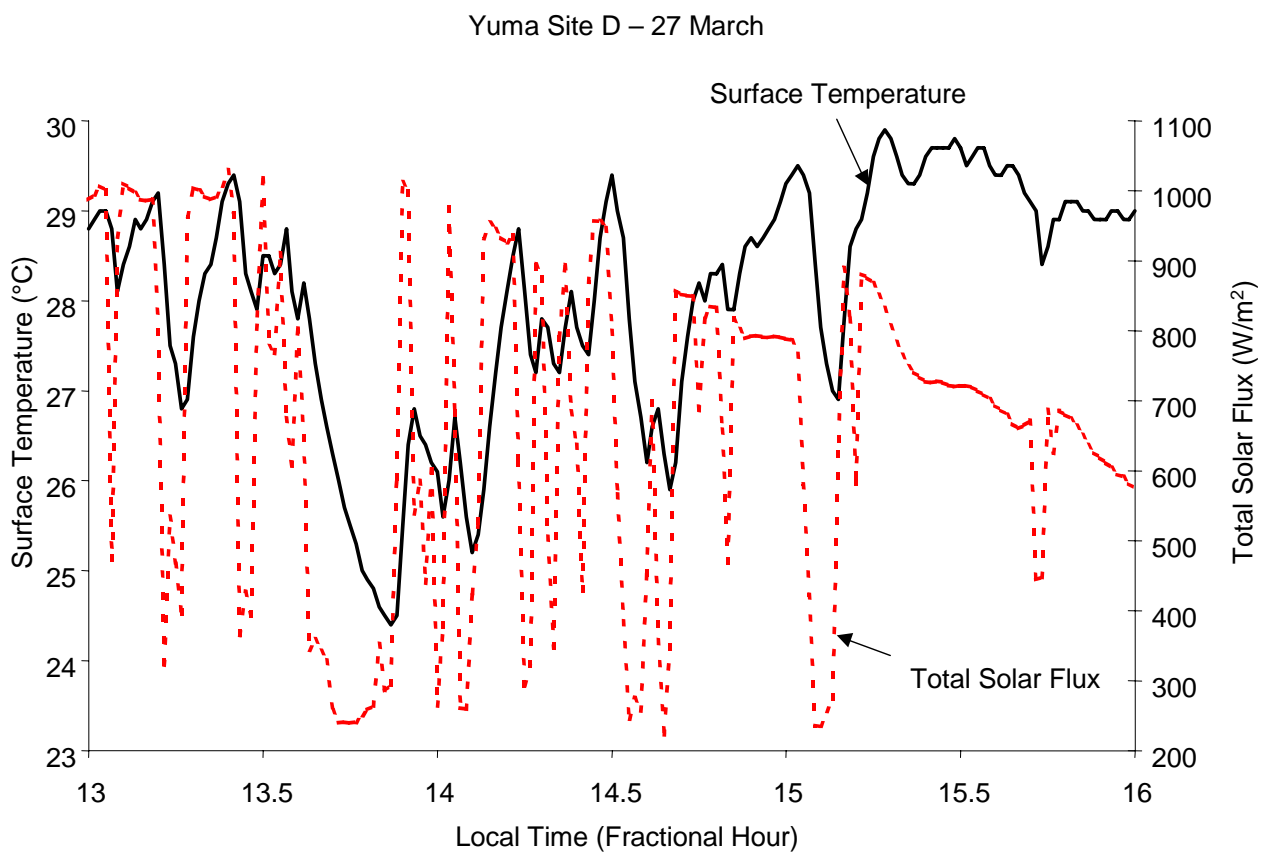

Figure 17. Solar flux and surface temperature for site D, 27 March, Yuma.

In a 12-min period between 1337 (13.6) and 1349 (13.8) the surface temperature dropped $3.8^{\circ} \mathrm{C}$ and the solar flux changed from approximately $900 \mathrm{~W} / \mathrm{m}^{2}$ to approximately $240 \mathrm{~W} / \mathrm{m}^{2}$. These fluctuations in the total solar flux are associated with partly cloudy sky conditions. In dry, desert-like climates, the surface temperature responds rapidly to changes in solar loading. Figure 18 depicts the spatial and temporal variability in the surface temperature and the total solar flux for sites C and D. Site C was located in an area characterized by a hard-packed, darkcolored surface. Site D was located in the same area, but it was approximately $100 \mathrm{~m}$ south of site $\mathrm{D}$. The ratio of the surface temperature at site $\mathrm{C}$ to that at site $\mathrm{D}$ is less than 1, for the 3-hr period depicted in Figure 18. It is believed this is due to differences between the two sites (slope, vegetation cover, soil type, etc.). The ratio of the total solar flux for the two sites is also depicted in this figure. At times this ratio has values as high as 2, indicating the total solar flux at one site is twice that at the other site, even though the two sites are only about $100 \mathrm{~m}$ apart. There are also periods when the ratio is 1 . An inspection of the data reveals that most of these periods correspond to clear conditions. For the most part, the surface temperature ratio is in phase with the solar flux ratio (there is a small time 
lag). Two exceptions are around 1330 (13.5) and after 1530 (15.5). During the first period the solar fluxes at both sites are on the order of $250 \mathrm{~W} / \mathrm{m}^{2}$, indicating there is little or no direct component of solar flux. During the second period, conditions are clear and the total solar flux consists of both the direct and diffuse component.

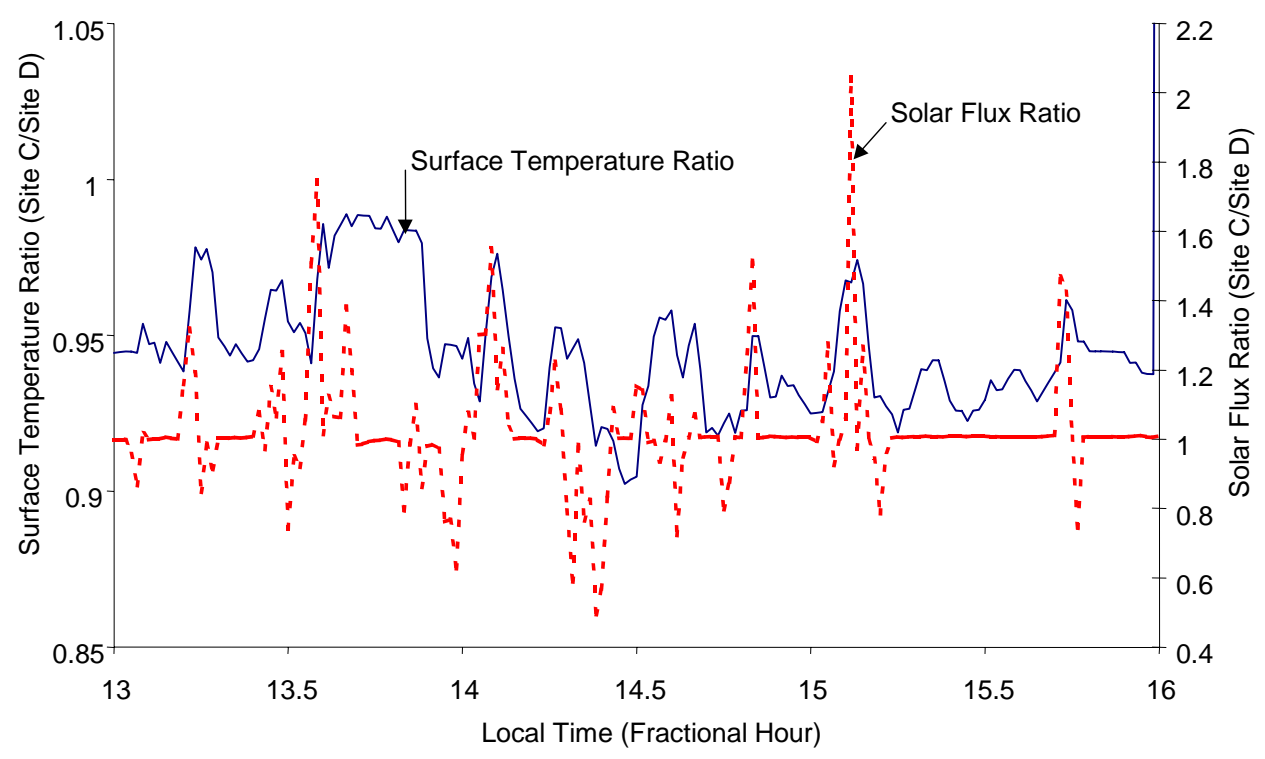

Figure 18. Surface temperature and solar flux ratio for sites $C$ and $D$.

While it cannot be verified, it is believed that the variations in the surface temperature ratio during these periods reflect, in part, variations in the other components of the energy balance.

\section{Grayling I: Partly cloudy skies}

During the daylight hours, the cloud cover varied from nine-tenths coverage during the early morning to one-tenth coverage in the late afternoon.

The distribution of the total solar flux for both the measured values from site E1 and E2 and the AIM-calculated values is presented in Figure 19.

The measured values are the 1-min values for the daylight period for locations E1 and E2. The AIM values are the values calculated for an 8- by 8-km area based on the cloud amounts reported on the hour. Thus, the AIM values are a snapshot over an 8- by 8-km aerial extent on the hour, while the measured values are the minute-by-minute values for a single location. AIM has a higher percentage of the flux values in the lower bin ranges. Even the measured values from 
two sites, separated by approximately $100 \mathrm{~m}$, show significant differences in the percent occurrence of flux values. On minute-by-minute bases, the shadowing at the two sites varies significantly.

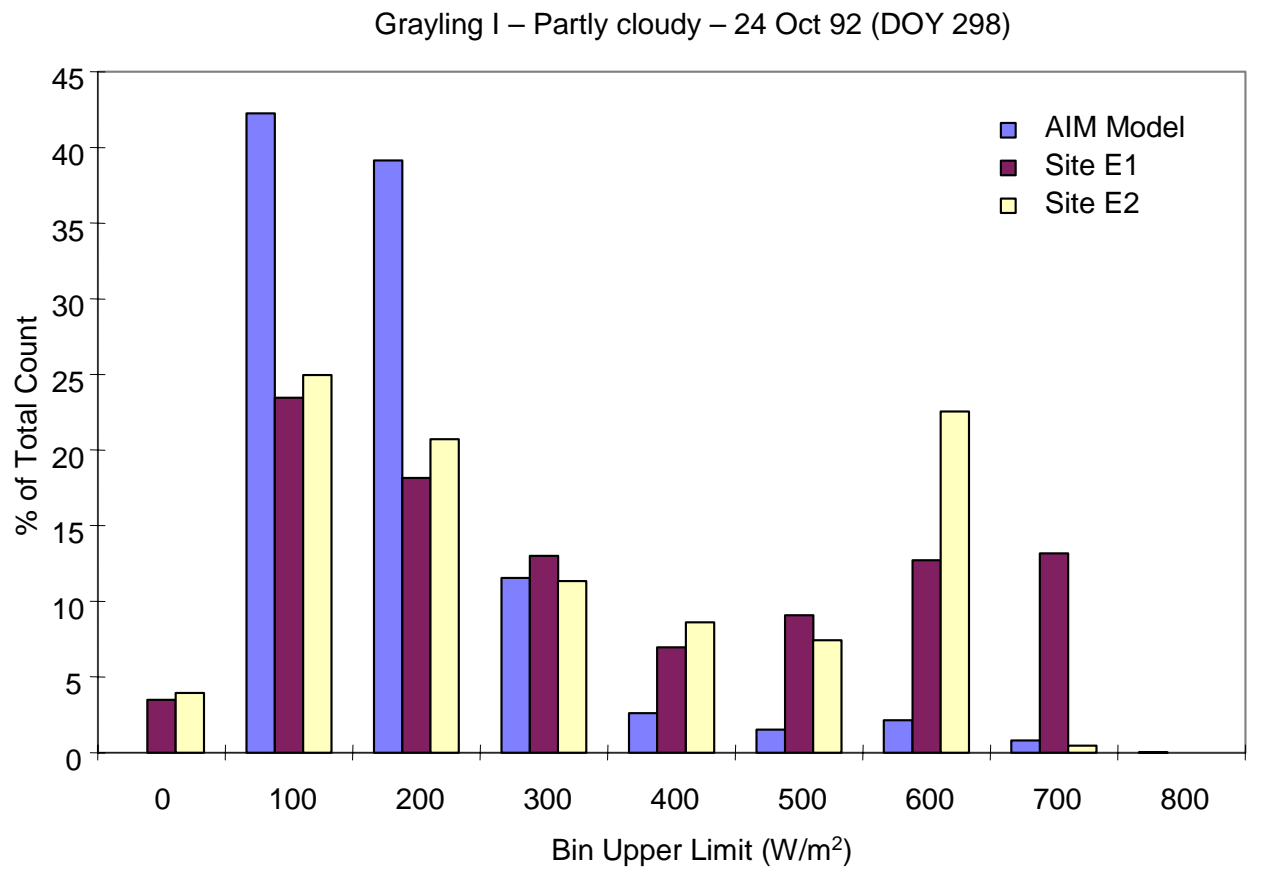

Figure 19. Distribution of total solar flux for sites E1 and E2 for Grayling I and AIM-calculated values.

From Table 7 it is evident that the hourly reported cloud cover changed during the day. In general, there was a clearing trend. Potentially, the AIM distribution of flux values may more closely resemble the measured values if the model was run more frequently. Unfortunately, it is not possible to do this since cloud observations were not taken more frequently than once per hour. These flux values along with the flux values computed from Shapiro's technique and MODTRAN have been used to ascertain the response of the surface temperature. Figure 20 presents the results for bare ground, and Table 8 summarizes the results for all surfaces. As anticipated, the surface temperatures calculated using the AIM maximum and minimum values of solar flux for each hour bracket the other values. As pointed out earlier, we would not anticipate experiencing this range of temperatures because a single spatial location would not experience either the minimum or the maximum solar value for the entire day. What it does indicate is that we can experience a range of temperatures at a single location due to variations in solar loading. In fact, the range of temperature differences 
exceeds the stated accuracy of the thermal model. In modeling synthetic scenes, it is important either to model accurately the solar flux values or to state the results in terms of a potential temperature range rather than a single value.

\begin{tabular}{|c|c|c|c|}
\hline \multirow[b]{2}{*}{ Time } & \multicolumn{3}{|c|}{ Cloud amount (tenths) } \\
\hline & Low & Middle & High \\
\hline 6 & 0.0 & 0.0 & 0.0 \\
\hline 7 & 0.0 & 0.6 & 0.0 \\
\hline 8 & 0.7 & 0.1 & 0.0 \\
\hline 9 & 0.9 & 0.0 & 0.0 \\
\hline 10 & 0.9 & 0.0 & 0.0 \\
\hline 11 & 0.8 & 0.0 & 0.0 \\
\hline 12 & 0.6 & 0.0 & 0.0 \\
\hline 13 & 0.6 & 0.0 & 0.0 \\
\hline 14 & 0.3 & 0.0 & 0.0 \\
\hline 15 & 0.3 & 0.0 & 0.0 \\
\hline 16 & 0.1 & 0.0 & 0.0 \\
\hline 17 & 0.1 & 0.0 & 0.1 \\
\hline 18 & 0.1 & 0.0 & 0.1 \\
\hline 19 & 0.1 & 0.0 & 0.2 \\
\hline 20 & 0.1 & 0.0 & 0.2 \\
\hline
\end{tabular}

Yuma: Partly cloudy skies

The variations in the cloud cover are given in Table 9. Maximum cloud cover occurs around solar noon.

In Figure 21, the distribution of the AIM total solar flux at 1300 local is depicted. As indicated in Table 9, the total cloud amount at 1300 local is $70 \%$. Flux values greater than $1000 \mathrm{~W} / \mathrm{m}^{2}$ cover only $7 \%$ of the 8 - by $8-\mathrm{km}$ area at 1300 local, but $51 \%$ of the area has flux values between 500 and $1000 \mathrm{~W} / \mathrm{m}^{2}$, with the majority of these values in the 500 to $600 \mathrm{~W} / \mathrm{m}^{2}$ range. Flux values in the 400 to $500 \mathrm{~W} / \mathrm{m}^{2}$ range cover another $22 \%$ of the area. In this dry climate, the surface temperature will respond rapidly to the wide range of flux values. As indicated earlier, the measured surface temperature changed approximately $4.0^{\circ} \mathrm{C}$ in just 12 minutes. 


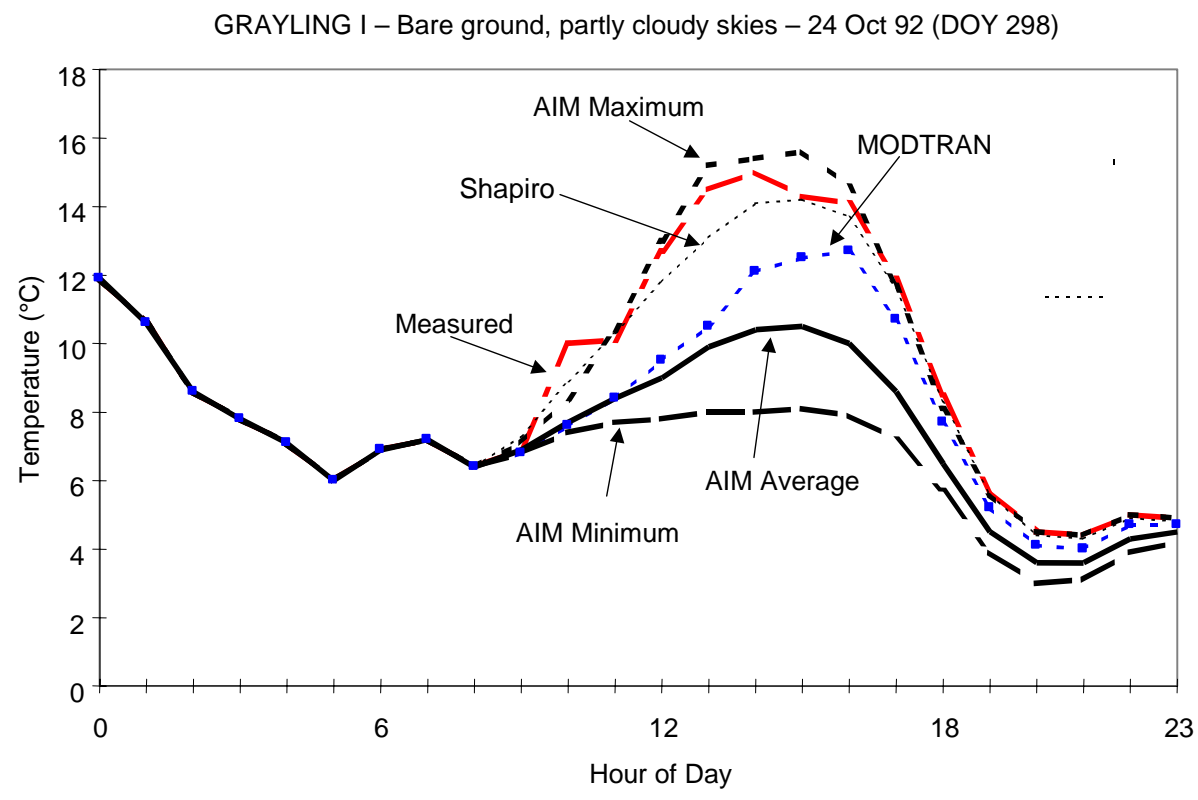

Figure 20. Surface temperature for bare ground resulting from different solar flux initialization schemes.

\begin{tabular}{|c|c|c|c|c|c|c|}
\hline \multirow[b]{2}{*}{ Measured } & \multicolumn{6}{|c|}{ Surface conditions } \\
\hline & & Bare & Snow & Deciduous & Coniferous & Grass \\
\hline \multirow[b]{3}{*}{ MODTRAN } & Maxdif & 4.0 & 2.6 & 1.9 & 1.3 & 1.3 \\
\hline & Mindif & 0.0 & 0.0 & 0.0 & 0.0 & 0.0 \\
\hline & Avgdif & 1.5 & 1.0 & 0.9 & 0.6 & 0.5 \\
\hline \multirow[b]{3}{*}{ Shapiro } & Maxdif & 1.4 & 0.9 & 1.0 & 0.7 & 0.5 \\
\hline & Mindif & -0.4 & -0.4 & -0.2 & -0.1 & -0.1 \\
\hline & Avgdif & 0.4 & 0.2 & 0.4 & 0.3 & 0.1 \\
\hline \multirow{3}{*}{$\begin{array}{c}\text { AlM } \\
\text { Average }\end{array}$} & Maxdif & 4.6 & 3.2 & 2.5 & 1.5 & 1.5 \\
\hline & Mindif & 0.0 & 0.0 & 0.0 & 0.0 & 0.0 \\
\hline & Avgdif & 2.3 & 1.8 & 1.0 & 0.6 & 0.7 \\
\hline \multirow{3}{*}{$\begin{array}{c}\text { AIM } \\
\text { Maximum }\end{array}$} & Maxdif & 1.6 & 1.2 & 0.9 & 0.5 & 0.6 \\
\hline & Mindif & -1.3 & -0.9 & -0.4 & -0.3 & -0.5 \\
\hline & Avgdif & -0.1 & 0.0 & 0.0 & 0.0 & 0.0 \\
\hline \multirow{3}{*}{$\begin{array}{c}\text { AlM } \\
\text { Minimum }\end{array}$} & Maxdif & 7.0 & 5.3 & 3.7 & 2.1 & 2.4 \\
\hline & Mindif & 0.0 & 0.0 & 0.0 & 0.0 & 0.0 \\
\hline & Avgdif & 3.3 & 2.7 & 1.5 & 0.8 & 1.1 \\
\hline
\end{tabular}


Table 9. Cloud amounts used in solar flux models.

\begin{tabular}{|c|c|c|c|}
\hline \multirow{2}{*}{ Time } & \multicolumn{3}{|c|}{ Cloud amount (tenths) } \\
\cline { 2 - 4 } & Low & Middle & High \\
\hline 7 & 0.2 & 0.0 & 0.0 \\
8 & 0.5 & 0.0 & 0.0 \\
9 & 0.0 & 0.0 & 0.0 \\
10 & 0.0 & 0.0 & 0.0 \\
\hline 11 & 0.1 & 0.0 & 0.0 \\
12 & 0.1 & 0.0 & 0.0 \\
13 & 0.7 & 0.0 & 0.0 \\
14 & 0.6 & 0.0 & 0.0 \\
15 & 0.7 & 0.0 & 0.0 \\
\hline 16 & 0.4 & 0.0 & 0.2 \\
17 & 0.3 & 0.0 & 0.3 \\
18 & 0.1 & 0.1 & 0.1 \\
\hline
\end{tabular}

Yuma I - 27 Mar 93 (DOY 086) 1300 hr - Partly cloudy

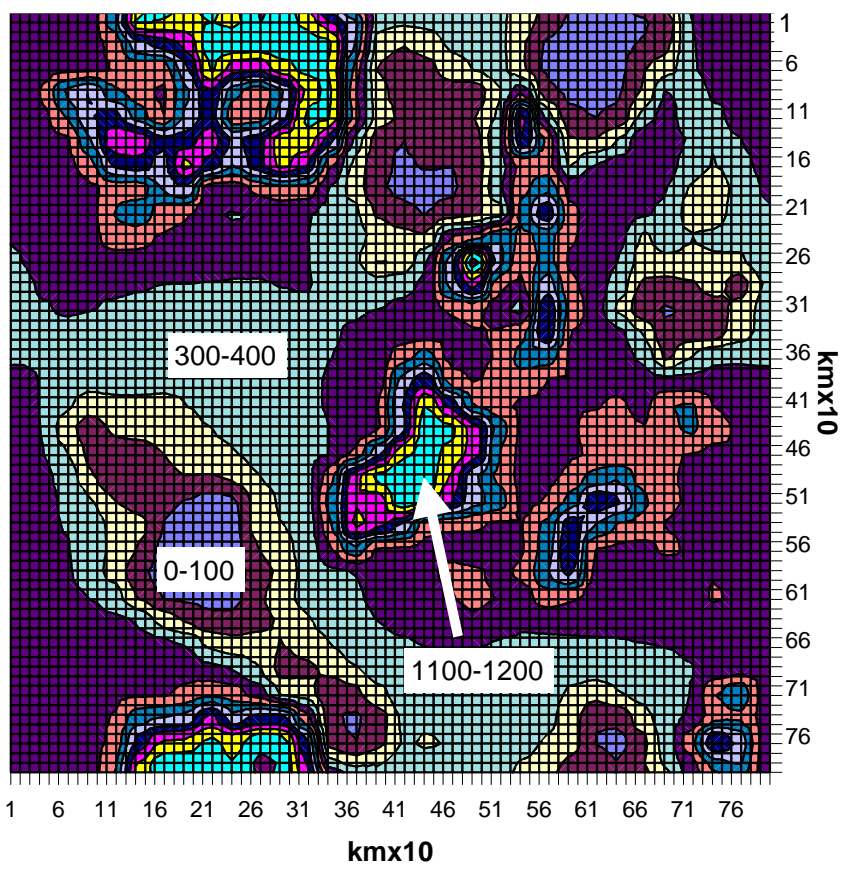

- 1300.0-1400.0

- 1200.0-1300.0

1100.0-1200.0

1000.0-1100.0

c 900.0-1000.0

- 800.0-900.0

$700.0-800.0$

600.0-700.0

500.0-600.0

- 400.0-500.0

300.0-400.0

200.0-100.0

- 100.0-200.0

$0.0-100.0$

Figure 21. Distribution of total solar flux at 1300 local as calculated by AIM model. 


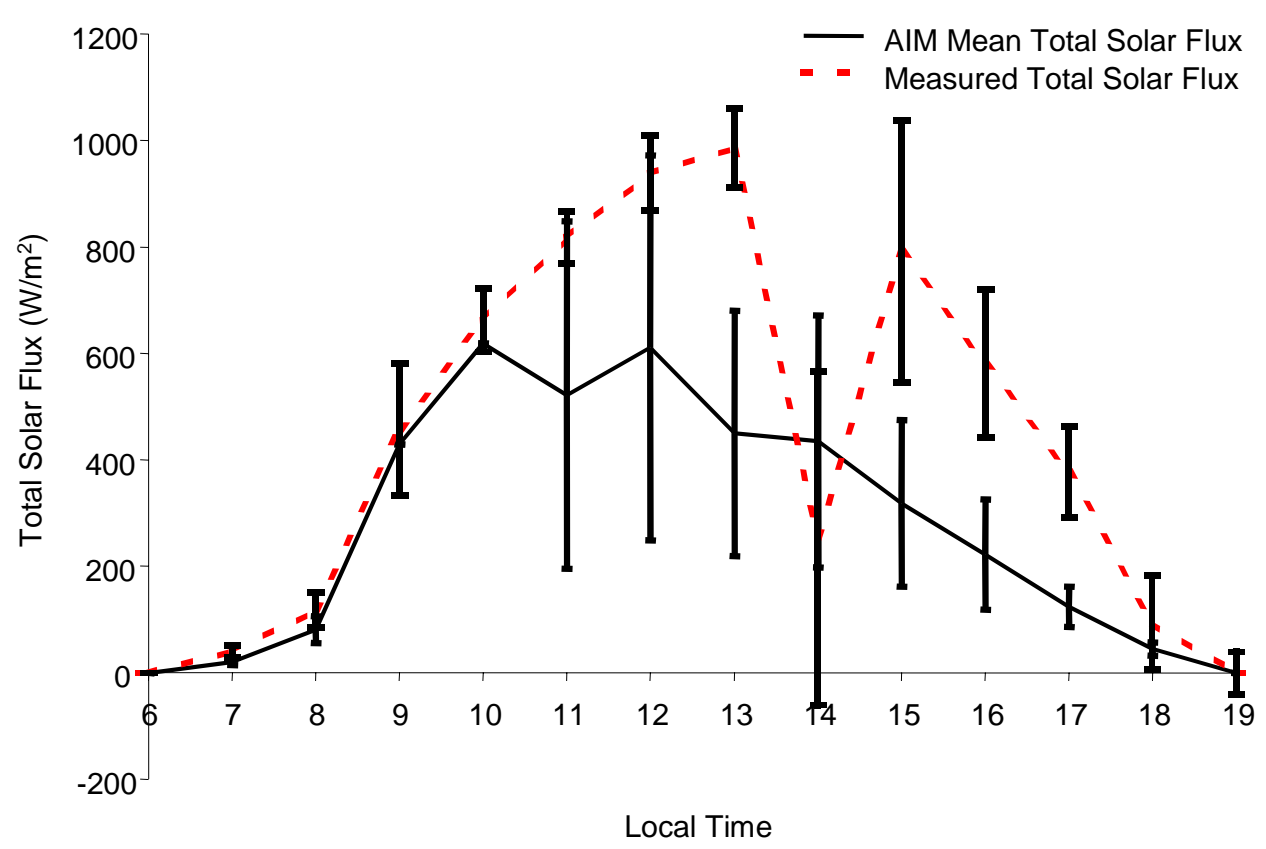

Figure 22. Mean AIM-calculated total solar flux \pm one standard deviation.

The standard deviation associated with the temporal variation of the measured flux and the spatial variation of the AIM flux values is given in Figure 22. For the AIM total solar flux, this standard deviation is based on the 6400 values in the 8- by 8-km area, and for the measured value it is the deviation associated with the 60 previous 1-min values. The standard deviation at 0900 and 1000 local is 0 for the AIM values since there is no cloud cover at these times. The largest standard deviation for both the measured data and the AIM calculated values occurs at 1400 local. The standard deviation associated with the temporal variation (measured data) is approximately $313 \mathrm{~W} / \mathrm{m}^{2}$; the standard deviation associated with the spatial variability (AIM calculated values) is approximately 263 $\mathrm{W} / \mathrm{m}^{2}$. If we assume no evolution in the cloud geometry, then there should be a relationship between the spatial and temporal variation of the solar flux. At this point in time we do not have sufficient information to establish this relationship, and the information in Figure 22 is presented to emphasize the spatial and temporal variability of the solar flux for partly cloudy conditions and the resulting impact on the surface temperature. The spatial and temporal variations in the surface temperature represent clutter to an infrared sensor system and may reduce the performance of that system. The impact of the different initialization schemes on a vegetated surface is depicted in Figure 23. The resulting surface temperature from all schemes tends to follow the surface temperature as predicted by using the measured fluxes. In addition, the range of vegetation temperature values as 
predicted from using the maximum and minimum is not as great as for bare ground (Table 10). The average difference between the surface temperature computed using the measured flux and the surface temperature using the AIM maximum flux is $-3.1^{\circ} \mathrm{C}$, indicating AIM maximum fluxes result in warmer surface temperatures. The average difference using the AIM minimum flux is $4.6^{\circ} \mathrm{C}$. Thus, the range of surface temperatures based on the average of the temperatures computed using the maximum and minimum AIM fluxes is $7.7^{\circ} \mathrm{C}$.

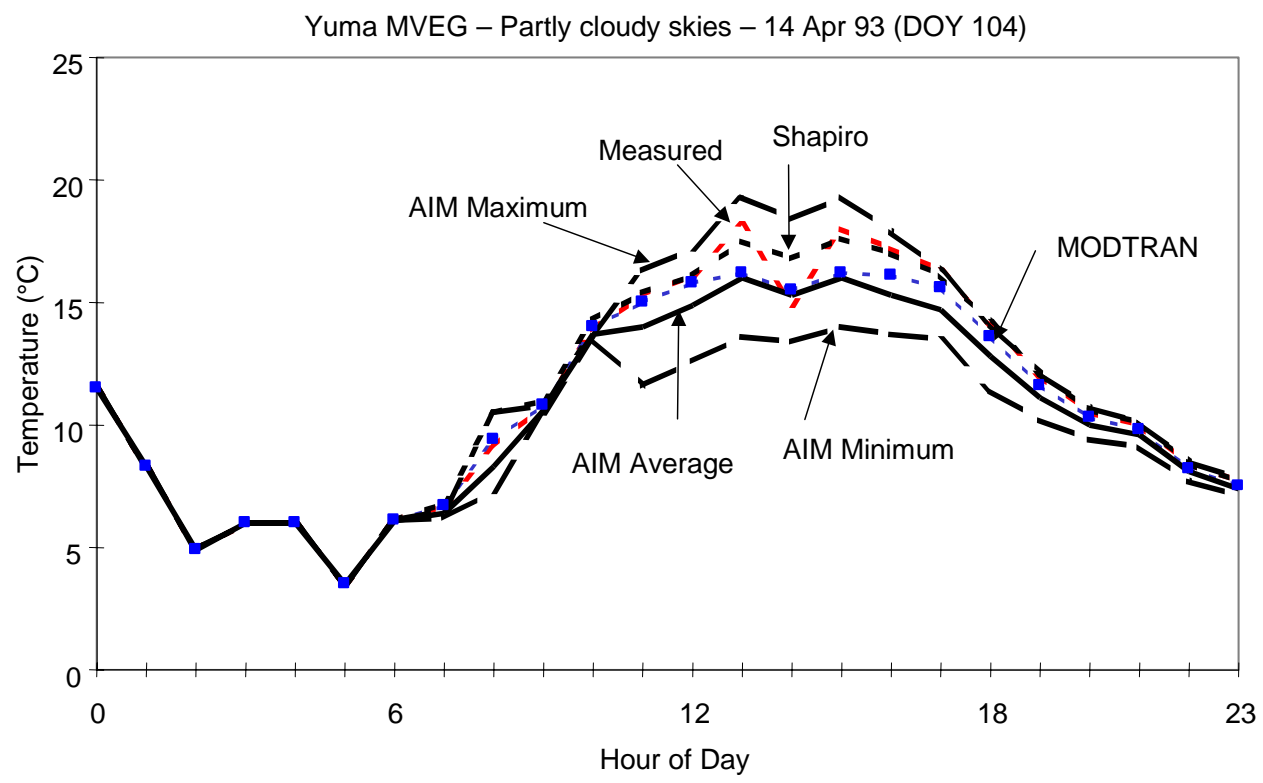

Figure 23. Surface temperature for medium vegetation (grass) for different solar flux initialization schemes.

During the 1300 to 1400 local time period, the measured surface temperature difference between sites $\mathrm{C}$ and $\mathrm{D}$ was as high as $2.0^{\circ} \mathrm{C}$. These sites are separated by less than $100 \mathrm{~m}$. During the night, the surface temperature difference is on the order of 0.2 to $0.3^{\circ} \mathrm{C}$. The differences during the 1300 to 1400 local time period are believed to be due to differential solar loading resulting from the partly cloudy conditions.

\section{Grayling II: Partly cloudy skies}

Before noon local time, sky conditions ranged from partly cloudy to overcast. During the afternoon, skies cleared. The changes in the cloud conditions and their impact on the total solar flux and the surface temperature are given in Table 11 and Figure 24, respectively. 
Table 10. Summary of surface temperature differences $\left({ }^{\circ} \mathrm{C}\right)$ computed using different solar flux initialization schemes and surface conditions.

\begin{tabular}{|c|c|c|c|}
\hline \multirow{2}{*}{ Measured } & \multicolumn{3}{|c|}{ Surface conditions } \\
\cline { 2 - 4 } & & Bare & Grass \\
\hline \multirow{3}{*}{ MODTRAN } & Maxdif & 5.8 & 2.0 \\
& Mindif & -1.4 & -0.6 \\
& Avgdif & 0.9 & 0.3 \\
\hline Shapiro & Maxdif & 2.0 & 0.7 \\
& Mindif & -5.2 & -1.9 \\
& Avgdif & -0.3 & -0.1 \\
\hline AlM & Maxdif & 6.5 & 2.2 \\
Average & Mindif & -0.5 & -0.4 \\
& Avgdif & 1.8 & 0.6 \\
\hline AIM & Maxdif & 0.4 & 0.2 \\
Maximum & Mindif & -10.1 & -3.5 \\
& Avgdif & -1.3 & -0.4 \\
\hline AlM & Maxdif & 14.7 & 4.6 \\
Minimum & Mindif & 0.0 & 0.0 \\
\hline
\end{tabular}

The total solar flux during the morning varies by a factor of three over relatively short time periods. Not only does the surface temperature follow the general trend of the solar flux but it also reflects some of the high-frequency changes in the solar flux. Changes in surface temperature occur on the order of several degrees. At 1000 local, the sky conditions were basically overcast with low cloud. Under these conditions, AIM calculated total solar flux values in a narrow distribution with values that are typical of diffuse solar radiation (Figure 25). On the other hand, the measured values are considerably higher and spread over a greater range. The AIM fluxes are spatially distributed fluxes based on the cloud cover at a single time, and the measured fluxes are temporally distributed over the previous 60 minutes (60 observations). In this case, it appears the spatial distribution cannot be equated to the temporal distribution.

The AIM-calculated total solar flux in Figure 26 is the average for the 6400 values for the 8- by 8-km area based on the cloud amount on the hour. In general, the AIM total solar flux is less during cloud periods, but rapidly converges to the other values when the skies clear after 1500 local. The spatial distribution of the clouds is based on CSSM. CSSM does have a capability to produce the temporal variation of clouds for short periods of time (on the order of $15 \mathrm{~min}$ ) but running AIM for minute-by-minute cloud distributions would be computation-prohibitive 
at this time. Accurate high-resolution modeling requires both the spatial and temporal distribution of clouds and the associated solar fluxes. The calculated surface temperature based on the different initialization schemes is given in Figure 27. In the afternoon when skies clear, the surface temperatures computed using the different initialization schemes are almost identical. Earlier in the day when it is cloudy this is not the case. In fact, the temperature difference is as large as $10^{\circ} \mathrm{C}$ for the initialization schemes based on the maximum and minimum AIMcalculated flux. In general, the values given in Table 12 for the different initialization schemes and surface conditions are greater than the corresponding values for overcast and clear-sky conditions. Partly cloudy skies will result in large spatial and temporal variations in solar loading.

Table 11. Cloud amounts used in solar flux models.

\begin{tabular}{|c|c|c|c|}
\hline \multirow{2}{*}{ Time } & \multicolumn{3}{|c|}{ Cloud amount (tenths) } \\
\cline { 2 - 4 } & Low & Middle & High \\
\hline 6 & 0.1 & 0.2 & 0.7 \\
7 & 0.8 & 0.0 & 0.2 \\
8 & 1.0 & 0.0 & 0.0 \\
9 & 1.0 & 0.0 & 0.0 \\
10 & 1.0 & 0.0 & 0.0 \\
\hline 11 & 0.9 & 0.0 & 0.1 \\
12 & 0.6 & 0.0 & 0.4 \\
13 & 0.4 & 0.0 & 0.3 \\
14 & 0.2 & 0.0 & 0.2 \\
15 & 0.1 & 0.0 & 0.1 \\
\hline 16 & 0.0 & 0.0 & 0.0 \\
17 & 0.0 & 0.0 & 0.0 \\
18 & 0.0 & 0.0 & 0.0 \\
19 & 0.0 & 0.0 & 0.0 \\
20 & 0.0 & 0.0 & 0.0 \\
\hline
\end{tabular}




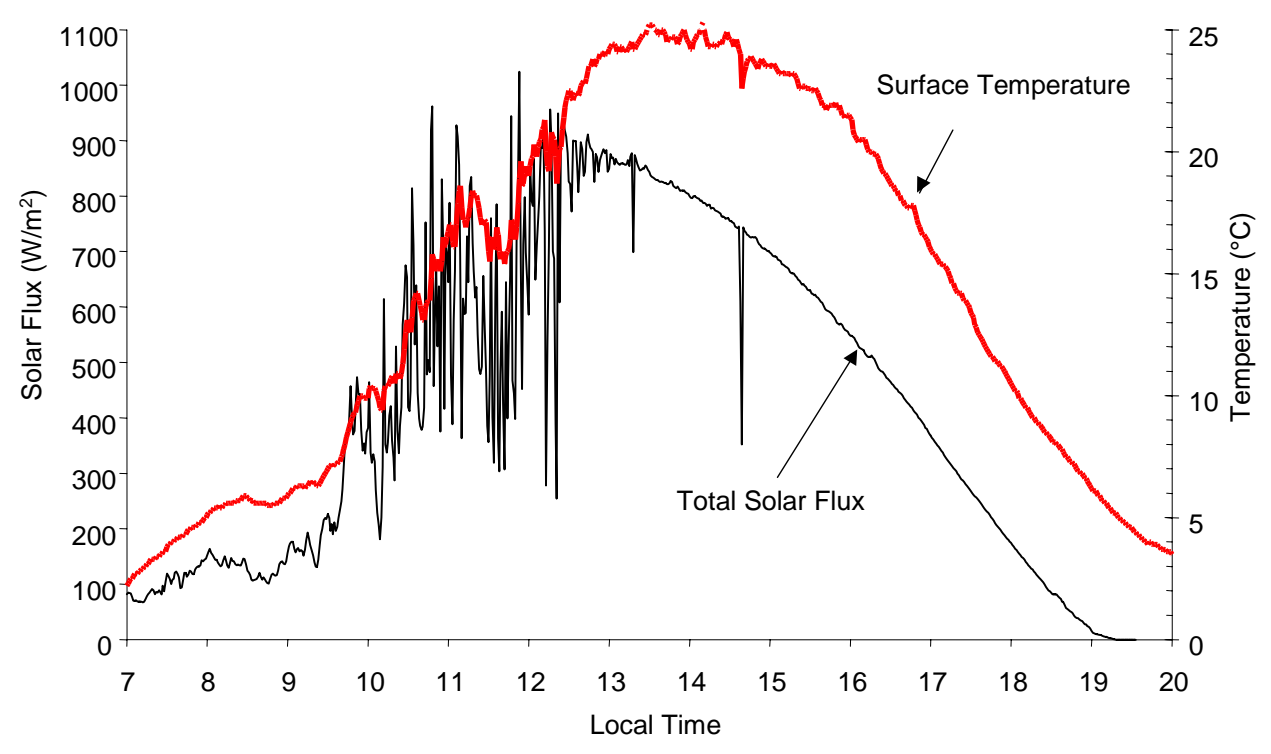

Figure 24. Total solar flux and surface temperature measurements at site E3 for partly cloudy conditions.

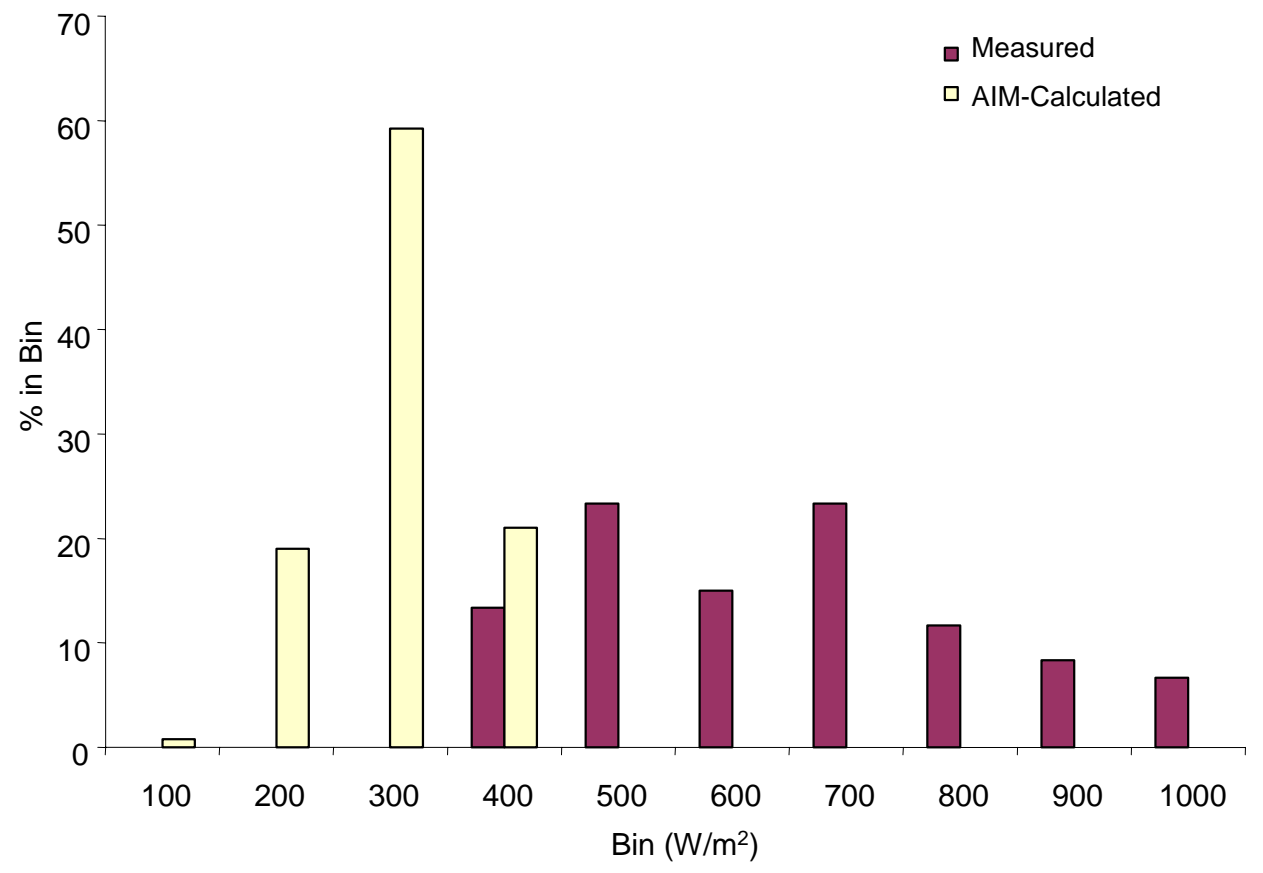

Figure 25. Distribution of measured total solar flux for period of 60 minutes for Grayling II and AIM-calculated values for 8- by 8-km area at 1000 local. 
Grayling II - 10 Apr 94 (DOY 100)

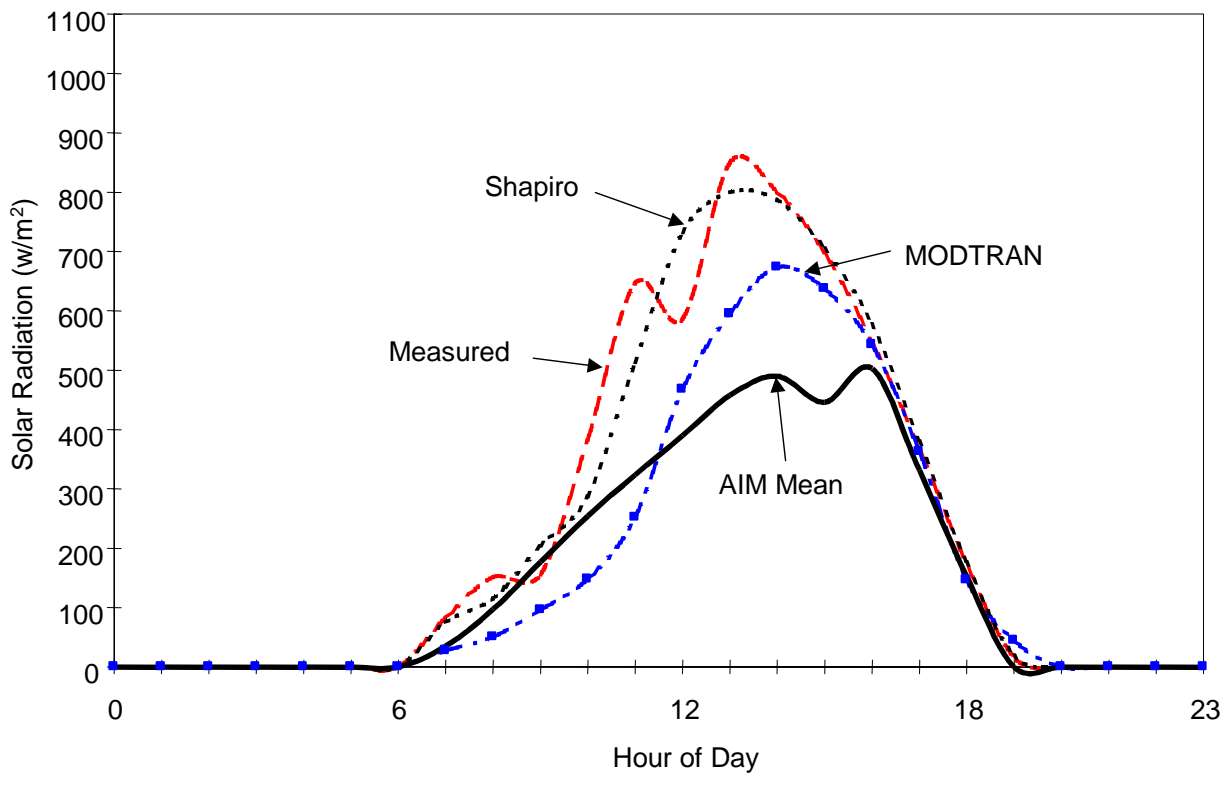

Figure 26. Total solar flux measured and calculated for partly cloudy conditions for Grayling II.

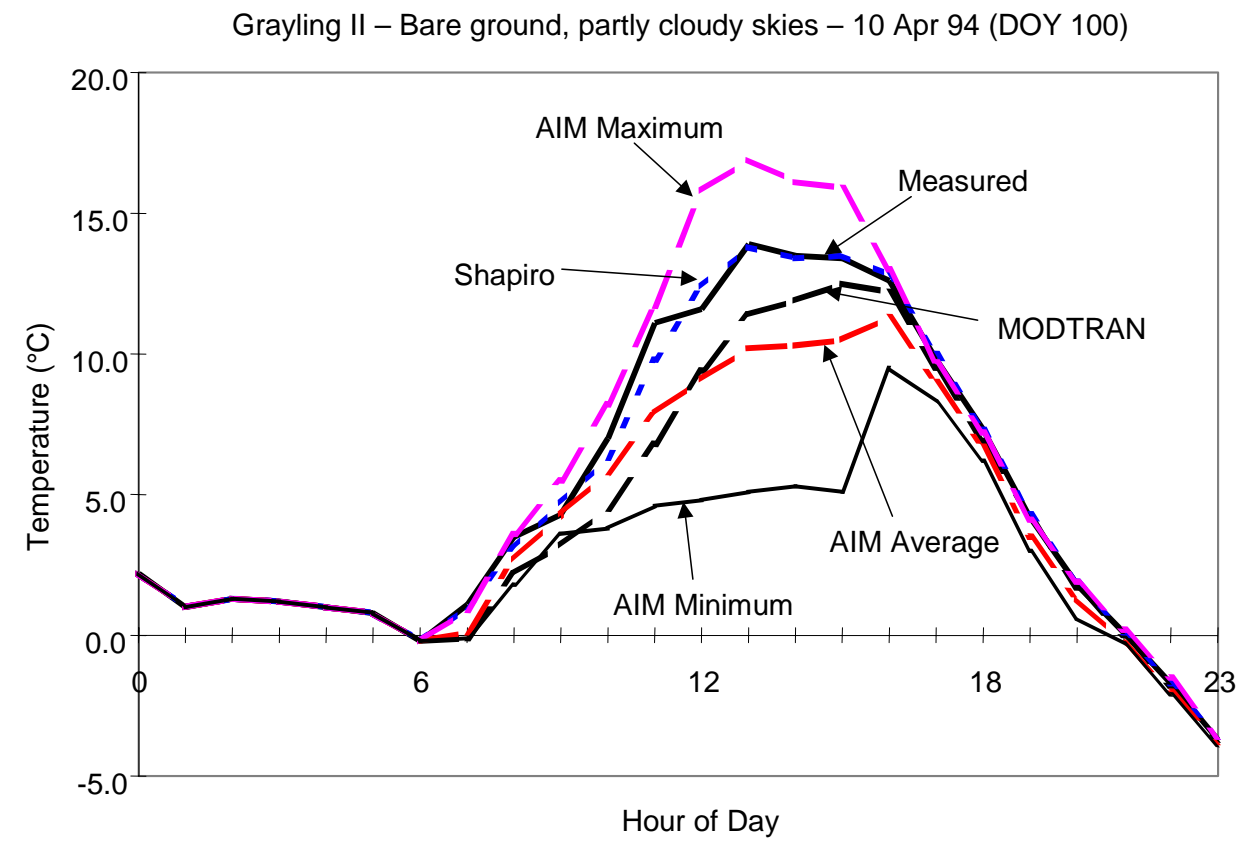

Figure 27. Calculated surface temperature based on different initialization schemes. 


\begin{tabular}{|c|c|c|c|c|c|c|}
\hline \multirow[b]{2}{*}{ Measured } & \multicolumn{6}{|c|}{ Surface conditions } \\
\hline & & Bare & Snow & Deciduous & Coniferous & Grass \\
\hline \multirow[b]{3}{*}{ MODTRAN } & Maxdif & 4.3 & 5.2 & 2.3 & 1.5 & 1.5 \\
\hline & Mindif & 0.0 & 0.0 & -0.4 & 0.0 & 0.0 \\
\hline & Avgdif & 0.8 & 0.5 & 0.4 & 0.2 & 0.2 \\
\hline \multirow[b]{3}{*}{ Shapiro } & Maxdif & 1.3 & 0.2 & 0.8 & 0.4 & 0.5 \\
\hline & Mindif & -0.8 & -0.2 & -0.4 & -0.3 & -0.4 \\
\hline & Avgdif & 0.0 & 0.0 & 0.0 & 0.0 & 0.0 \\
\hline \multirow{3}{*}{$\begin{array}{c}\text { AlM } \\
\text { Average }\end{array}$} & Maxdif & 3.7 & 5.2 & 2.2 & 1.2 & 1.4 \\
\hline & Mindif & 0.0 & 0.0 & 0.0 & -0.1 & 0.0 \\
\hline & Avgdif & 1.0 & 0.7 & 0.5 & 0.3 & 0.3 \\
\hline \multirow{3}{*}{$\begin{array}{c}\text { AlM } \\
\text { Maximum } \\
\end{array}$} & Maxdif & 0.2 & 0.2 & 0.1 & 0.1 & 0.0 \\
\hline & Mindif & -4.2 & -1.4 & -2.5 & -1.6 & -1.6 \\
\hline & Avgdif & -0.7 & -0.3 & -0.4 & -0.3 & -0.2 \\
\hline \multirow{3}{*}{$\begin{array}{c}\text { AlM } \\
\text { Minimum }\end{array}$} & Maxdif & 8.8 & 8.8 & 5.0 & 2.9 & 2.8 \\
\hline & Mindif & 0.0 & 0.0 & 0.0 & -0.1 & 0.0 \\
\hline & Avgdif & 2.3 & 1.7 & 1.2 & 0.7 & 0.7 \\
\hline
\end{tabular}




\section{DISCUSSION AND CONCLUSION}

Distributed energy budget models require distributed initialization conditions. The optimal response of snowmelt using the one-dimensional SNTHERM (the basic model used in SWOETHERM) model in a distributed mode for meteorological cell sizes ranging from $9 \mathrm{~km}$ to $72 \mathrm{~km}$ for the Sava River Basin was obtained using a cell size of $9 \mathrm{~km}$. IMETS is being designed to provide meteorological information over spatial extents as large as 500 - by $500-\mathrm{km}$ with a cell resolution of approximately $10 \mathrm{~km}$ (higher resolutions are being planned for the future). The response of the surface temperature for cloudy and partly cloudy conditions can be on the order of several degrees for temporal and spatial variations in the solar flux on the order of tens of minutes and several hundred meters. Of the solar flux models investigated, only AIM provides the spatial distribution of the solar flux. This model also has the potential to provide the temporal variations of the solar flux if it is coupled with a cloud model that generates both the spatial and temporal variation in cloud cover. CSSM has this capability. Run times of models like AIM that can provide spatial, temporal, and spectral information are an issue. We have investigated a number of techniques to decrease the run time of AIM, and additional acceleration techniques will be explored in the future. For clear-sky conditions and small spatial extents, semi-empirical models like Shapiro's model provide sufficiently accurate flux information. The issue becomes more complicated when we consider overcast or partly cloudy skies. Even for overcast conditions, we observed both spatial and temporal variations in the measured solar fluxes that resulted in surface temperature variations of several degrees. These variations in the solar flux have long been attributed to variations in the cloud optical properties (Welch and Wielicki 1984, Li et al. 1994). We have shown that as the aerosol optical depth increases, the direct component decreases in a nonlinear fashion and the diffuse component first increases and then decreases. We would expect a similar behavior for variations in cloud optical depths. This implies that in addition to knowing the horizontal distribution of clouds it is also necessary to know typical cloud optical depths.

As indicated, the aerosol optical depth can impact the surface solar flux. Frequently the visibility is used as a surrogate for the aerosol optical depth. What is really needed is the variation of the optical depth with height. For clear and partly cloudy conditions, the surface predicted temperature based on the solar flux predicted by Shapiro's model follows closely the values predicted using the measured fluxes. The predictions are not as accurate for cloudy conditions, and thick haze conditions were not studied. While Shapiro's technique does allow for different cloud types, it does not allow for variations in the cloud thickness 
(variations in the cloud optical depth). In addition, the predictions based on Shapiro's model are for a single location at a single time. This model does not provide for spatial variations in the solar flux.

Another potential issue with respect to Shapiro's model is the relationship between observer-collected cloud cover information and the model-computed surface solar flux. Due to automated analysis techniques being developed for satellite or lidar retrieval of cloud data, it may be that the availability of humanobserved cloud condition information will be at a premium in the future. In this case, questions need to be raised as to the relationship between Shapiro's coefficients and the actual cloud state. Shapiro's model appears to have a good skill level in inferring solar flux given observed cloud conditions. Hence, whatever correlations are present between observed conditions and observed fluxes are accurately being preserved through his model. Thus, the effects of clouds that are unobservable are being convolved into the model responses, and correlations between these unseen upper cloud layers are included in the model coefficients applied strictly to the lower layer effects. These effects might explain the model's (Shapiro's) superior performance in handling some of the cloudy cases. However, if the method of measuring cloud conditions changes, the Shapiro model would need to be altered, since the same correlations might not exist when different data acquisition methods are used. One area of active exploration might be to inquire, using a 3-D model, what cloud optical conditions would be required to produce specific outputs from the Shapiro model. These considerations could lead to extensions of the Shapiro model under various cloud conditions.

Spatial and temporal variations in the state of the ground can negatively impact weapon system performance. Sufficient spatial variation in the surface temperature can confound infrared target detection. And, as noted in the data analysis, often measurements taken less than a kilometer apart showed significant variations in the sensed incident fluxes. Variations in snowmelt conditions due to variations in solar loading can negatively impact active radar systems as well. Techniques are being developed to segment terrain in order to run one-dimensional energy budget models over aerial extents for tens of kilometers to portray the battlespace state of the ground and the impact of the environment on military systems. Accurate depiction of the state of the ground for synthetic scene simulations depends, in part, on the terrain segmentation techniques employed and the accuracy of the distributed meteorological information used as boundary conditions for the energy budget models that calculate the state of the ground. 


\section{REFERENCES}

Balick, L.R., R.K. Scoggins, and L.E. Link (1981) Inclusion of a simple vegetation layer in terrain temperature models for thermal IR signature prediction. IEEE Transactions on Geoscience and Remote Sensing, GE19(3):143-152.

Ball, G.H., and D.J. Hall (1965) Isodata, a novel method of data analysis and pattern classification. Stanford Research Institute Technical Report (NTIS AD699616), Stanford, California.

Berk, A., L.S. Bernstein, and D.C. Robertson (1989) MODTRAN: A moderate resolution model for LOWTRAN 7. GL-TR-89-0122.

Cianciolo, M.E., and R.G. Rasmussen (1996) Cloud scene simulation modeling: The enhanced model—Final report. TR-6042-2. Hanscom Air Force Base, Massachusetts: Phillips Laboratory Atmospheric Structure Branch.

Davis, R.E., J.C. McKenzie, and J.P. Hardy (1998) Segmentation of geospatial data for spatially distributed modeling of snow. EOS Transactions, 79(45): F271.

Fu, Q., and K.N. Liou (1992) On the correlated $k$-distribution method for radiative transfer in nonhomogeneous atmospheres. Journal of Atmospheric Science, 49: 2139-2156.

Jordan, R. (1991) A one-dimensional temperature model for a snow cover: Technical documentation for SNTHERM.89. U.S. Army Cold Regions Research and Engineering Laboratory, Hanover, New Hampshire, Special Report 91-16.

Lacis, A.A., and V. Oinas (1991) A description of the correlated $k$ distribution method for modeling nongray gaseous absorption, thermal emission, and multiple scattering in vertically inhomogeneous atmospheres. Journal of Geophysical Research, 96: 9027-9063.

Li, J., D.J.W. Geldart, and P. Chylek (1994) Perturbation solution of 3D radiative transfer in a horizontally periodic inhomogeneous cloud field. Journal of Atmospheric Science, 51: 2110-2122.

NOAA SOLMET (1979) Hourly solar radiation-surface meteorological observations. Final Report D-9724. Asheville, North Carolina: National Climatic Center.

O’Brien, S.G., and D.H. Tofsted (1998) Physics-based visualization of dense natural clouds. II: Cloud-rendering algorithm. Applied Optics, 37: 7680-7688.

Passner, J.E. (1998) Cloud analysis and forecasts for IMETS. In Proceedings of the 16th Conference on Weather Analysis and Forecasting, 11-16 January 1998, Phoenix, Arizona, p. 478-480. 
Shapiro, R. (1972) Simple model for the calculation of the flux of solar radiation through the atmosphere. Applied Optics, 11: 760-764.

Shapiro, R. (1982) Solar radiative flux calculations from standard surface meteorological observations. AFGL-TR-82-0039. Hanscomb Air Force Base, Massachusetts: Air Force Geophysics Laboratory.

Shapiro, R. (1987) A simple model calculation of the flux of direct and diffuse solar radiation through the atmosphere. AFGL-TR-0200. Hanscomb Air Force Base, Massachusetts: Air Force Geophysics Laboratory.

Smith, J.A., K.J. Ranson, D. Nguyen, L.K. Balick, L.E. Link, L. Fritchen, and B.A. Hutchison (1981) Thermal vegetation canopy model studies. Remote Sensing of the Environment, 11: 311-326.

Tofsted, D.H., and S.G. O’Brien (1998) Three-dimensional radiative transfer modeling of tropospheric atmospheres. ARL-TR-1629. White Sands Missile Range, New Mexico: Army Research Laboratory.

Welch, R.M., and B.A. Wielicki (1984) Stratocumulus cloud field reflected fluxes: The effect of cloud shape. Journal of Atmospheric Science, 41: 30853103. 


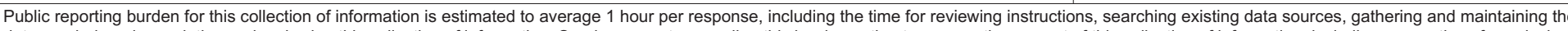

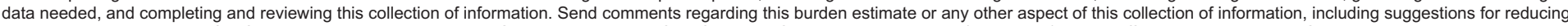

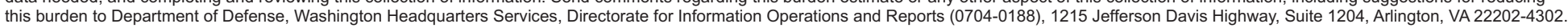

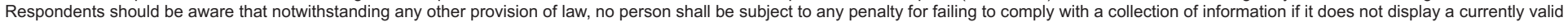
OMB control number. PLEASE DO NOT RETURN YOUR FORM TO THE ABOVE ADDRESS.
1. REPORT DATE (DD-MM-YY)
2. REPORT TYPE
3. DATES COVERED (From - To)

August 2003

Technical Report

4. TITLE AND SUBTITLE

5a. CONTRACT NUMBER

Solar Flux Initialization Schemes for

5b. GRANT NUMBER

Distributed Surface Energy Budget Modeling

5c. PROGRAM ELEMENT NUMBER

6. AUTHOR(S)

5d. PROJECT NUMBER

George G. Koenig and David H. Tofsted

5e. TASK NUMBER

5f. WORK UNIT NUMBER

7. PERFORMING ORGANIZATION NAME(S) AND ADDRESS(ES)

8. PERFORMING ORGANIZATION REPORT

U.S. Army Engineer Research and Development Center

Cold Regions Research and Engineering Laboratory

72 Lyme Road

ERDC/CRREL TR-03-13

Hanover, NH 03755-1290

9. SPONSORING/MONITORING AGENCY NAME(S) AND ADDRESS(ES)

10. SPONSOR / MONITOR'S ACRONYM(S)

U.S. Army Modeling and Simulation Office

HQDA DCS G3 (DAMO-ZA)

400 Army Pentagon

Washington, DC 20314-1000

12. DISTRIBUTION / AVAILABILITY STATEMENT

Approved for public release; distribution is unlimited.

Available from NTIS, Springfield, Virginia 22161.

13. SUPPLEMENTARY NOTES

\section{ABSTRACT}

It is a well-established fact that the state of the ground is driven in a large part by the downwelling solar and IR fluxes. Models developed to predict the state of the ground depend critically on these fluxes for initialization. When measured solar and infrared fluxes are not available they must be computed. We have compared the ground temperatures as computed by the thermal model SWOETHERM using different solar flux initialization schemes. These initialization schemes used measured solar flux values obtained during the Smart Weapons Operability Enhancement (SWOE) field programs, and calculated solar flux values from a semi-empirical model (Shapiro's model), a plane parallel model (MODTRAN), and ARL's AIM (Atmospheric Illumination Module) model. We investigated the response of the surface temperature to different solar flux initialization schemes while all other environmental parameters were held constant. We found that for clear skies all schemes resulted in nearly identical surface temperatures. For partly cloudy and cloudy skies only the AIM model can mimic the spatial variability observed with the measured solar fluxes. The Cloud Scene Simulation Model (CSSM) was used to determine the spatial variability of the clouds. The cloud distributions were then used by AIM to produce the variations of the surface solar loading. CSSM also has the capability to produce the temporal variations in the cloud fields for short periods of time. Thus, it would be possible to use CSSM and AIM to produce the temporal and spatial variations in the solar loading. Models like AIM frequently incur a large computational burden. In order to reduce the computational burden associated with AIM we have implemented several new procedures. Distributed energy budget models used to predict the state of the ground require distributed environmental parameters for initialization. Many of these parameters can be obtained from mesoscale models such as MM5 or databases associated with programs such as IMETS. But, to our knowledge, none of these models or programs provide distributed solar or infrared fluxes, a key initialization parameter of energy budget models. Models like AIM linked to CSSM, or for that matter any model that provides information on the spatial and temporal distribution of atmospheric conditions, can be used to provide the spatial and temporal distribution of radiative fluxes.

\begin{tabular}{|c|c|c|c|c|c|}
\hline 15. SUBJECT TERMS & \multicolumn{2}{|c|}{$\begin{array}{l}\text { Computational efficiency } \\
\text { Infrared flux } \\
\text { Solar flux }\end{array}$} & \multicolumn{3}{|c|}{$\begin{array}{l}\text { Spatial and temporal flux distribution } \\
\text { State-of-the-ground energy budget models }\end{array}$} \\
\hline \multicolumn{3}{|c|}{ 16. SECURITY CLASSIFICATION OF: } & 17. LIMITATION OF & 18. NUMBER & 19a. NAME OF RESPONSIBLE PERSON \\
\hline a. REPORT & b. ABSTRACT & c. THIS PAGE & & & 19b. TELEPHONE NUMBER (include area code) \\
\hline $\mathrm{U}$ & $\mathrm{U}$ & $\mathrm{U}$ & $\mathrm{U}$ & 72 & \\
\hline
\end{tabular}

